\title{
Consumer acceptance of intervention strategies for healthy food choices
}

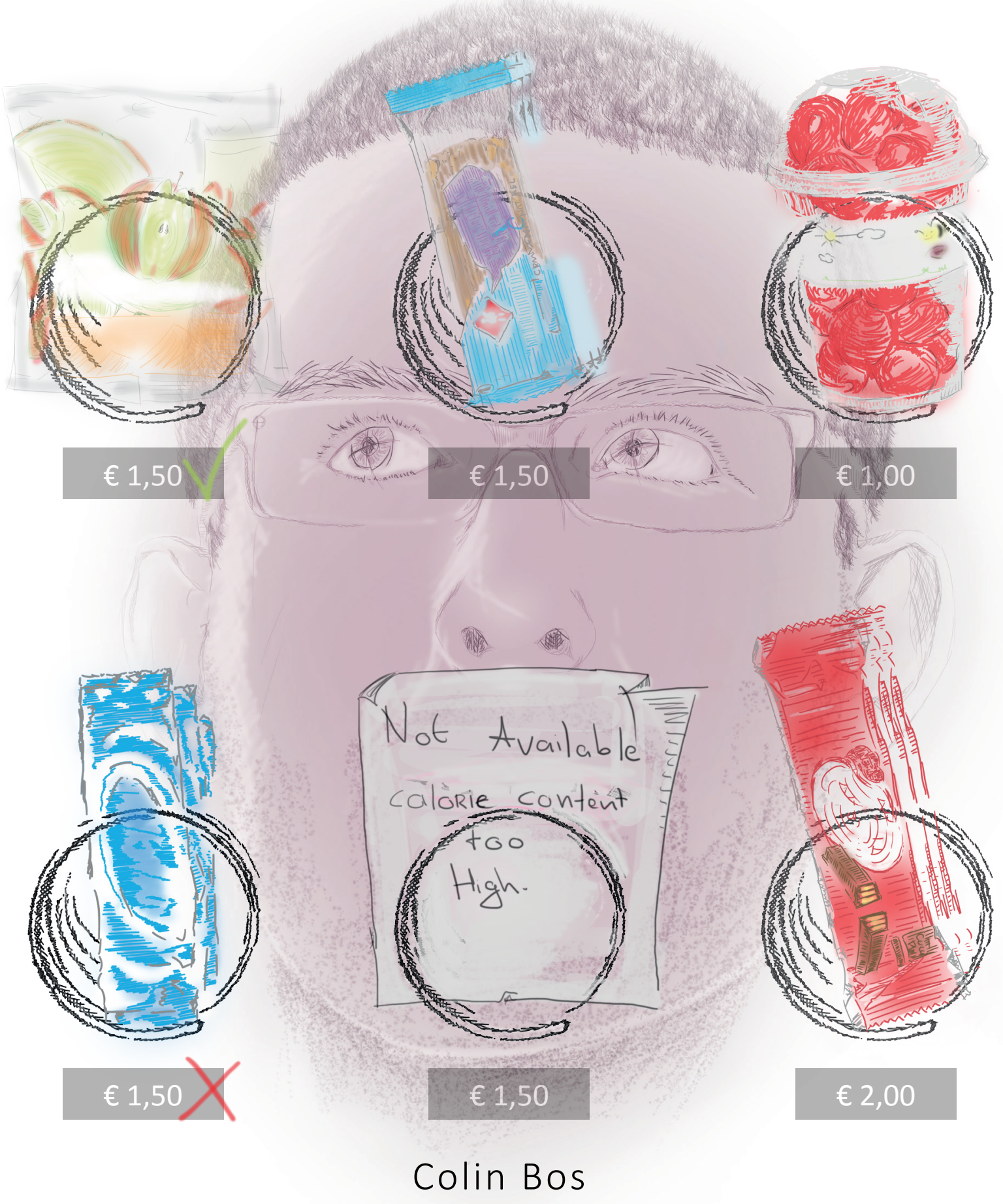



Consumer acceptance of intervention strategies for healthy food choices

Colin Bos 


\section{Thesis committee}

\section{Promotor}

Prof. Dr J.C.M. van Trijp

Professor of Marketing and Consumer Behaviour

Wageningen University

\section{Co-promotors}

Dr I.A.C.M. van der Lans

Assistant professor, Marketing and Consumer Behaviour Group

Wageningen University

Dr F.J. van Rijnsoever

Assistant professor, Innovation Studies

Utrecht University

\section{Other members}

Prof. Dr E. Kampman, Wageningen University

Prof. Dr K. van Ittersum, University of Groningen

Prof. Dr S. Kremers, Maastricht University

Dr M. Mazzocchi, University of Bologna, Italy

This research was conducted under the auspices of the Wageningen School of Social Sciences (WASS) 


\section{Consumer acceptance of intervention strategies for healthy food choices}

\section{Colin Bos}

\section{Thesis}

submitted in fulfilment of the requirements for the degree of doctor at Wageningen University

by the authority of the Rector Magnificus,

Prof. Dr A.P.J. Mol, in the presence of the

Thesis Committee appointed by the Academic Board to be defended in public on Tuesday 25 October 2016 at 1.30 p.m. in the Aula. 
Colin Bos

Consumer acceptance of intervention strategies for healthy food choices 170 pages.

PhD thesis, Wageningen University, Wageningen, NL (2016)

With references, with summary in English

ISBN 978-94-6257-912-5

DOI http://dx.doi.org/10.18174/389279 


\section{CONTENTS}

Chapter 1 General introduction

Chapter 2 Understanding consumer acceptance of intervention strategies for healthy food choices: a qualitative study

Chapter 3 Heterogeneity in barriers regarding the motivation, the opportunity and the ability to choose low-calorie snack foods and beverages: associations with real-life choices

Chapter 4 Consumer acceptance of population-level intervention strategies for healthy food choices: the role of perceived effectiveness and perceived fairness

Chapter 5 Promoting healthy choices from vending machines: effectiveness and consumer evaluations of four types of interventions

Chapter 6 General discussion

References

Summary

Dankwoord

About the author 

1

GENERAL INTRODUCTION 


\subsection{Background}

Detrimental dietary behaviours, both in terms of the quality and quantity of food consumption, are an important driver of overweight and obesity rates, which have increased dramatically over the past decades (Gortmaker et al., 2011; Lim et al., 2012). The obesity crisis has contributed to a worrying rise in the prevalence of noncommunicable diseases, particularly cardiovascular diseases, cancers, chronic respiratory diseases, and diabetes. Combined, these diseases account for $82 \%$ of the total of 38 million deaths attributable to non-communicable diseases each year (WHO, 2014).

While the world's population increased with $60 \%$ to 7.24 billion from 1980 to 2014, overweight (Body Mass Index (BMI) > 25) and obesity $(\mathrm{BMI}>30$ ) rates reached epidemic proportions by more than doubling to a total of 1.9 billion people (WHO, 2014). During that period, mean BMI increased by $0.4 \mathrm{~kg} / \mathrm{m}^{2}$ per decade (Finucane et al., 2011). Overweight, obesity and associated non-communicable diseases result in skyrocketing medical costs and also cost dearly in terms of quality of life (Withrow \& Alter, 2011).

Combatting overweight and obesity has become a bigger priority for governments and has also received more attention in the scientific world. Scholars and public health experts agree that to decrease overweight and obesity rates, environments that enable healthier dietary behaviours are indispensable (Nestle \& Jacobson, 2000; Swinburn et al., 2011). How these environments should enable healthier dietary behaviours, however, often sparks debates about the extent to which governments can/may intervene to achieve this (Kass, 2001; Ten Have, De Beaufort, Teixeira, Mackenbach, \& Van der Heide, 2011). The main goal of this thesis therefore will be to examine consumers' perspectives on promising avenues to enable healthier dietary behaviours.

\section{Causes of obesity}

Obesity can originate from a variety of causes, such as genes, health conditions and medicine use. The most prevalent cause of overweight and obesity, however, is a systematic imbalance between energy intake and energy expenditure (Swinburn et al., 2011). Continuous overconsumption makes the body store excessive calories as fat, leading to an increase in body weight (Hall et al., 2011). Neutralizing the energy imbalance can be done either by reducing energy intake or increasing energy expenditure. Since energy expenditure only decreased slightly during the last 
decades, the growth of obesity rates is primarily a result of excessive intake of energy (Shelley, 2012). Without downplaying the importance of increasing physical activity to combat overweight and obesity, the present thesis will focus on the consumption side of the obesity problem. The following section provides an overview of consumption-related drivers of the obesity epidemic.

From an evolutionary perspective, excessive calorie consumption makes perfect sense. In times of unstable food environments, where abundance and shortage alternated, eating high energy-dense foods decreased the risk of dying from malnutrition, thereby increasing the probability of survival (Bellisari, 2008). Such times of shortage, however, have disappeared in the Western world and generally foods are present in abundance, which eliminates the need for excessive calorie consumption. Therefore, nowadays, healthy dietary behaviour entails consuming mostly low-calorie, nutrient-dense products, whereas unhealthy consumption involves consuming primarily high-calorie products that are high in fat, sugar, and salt, and contain very little nutrients (Andrieu, Darmon, \& Drewnowski, 2006; Drewnowski, Monsivais, Maillot, \& Darmon, 2007; Drewnowski \& Specter, 2004). With this in mind, first drivers regarding the food system and subsequently individual characteristics that drive obesity will be discussed (Figure 1.1).

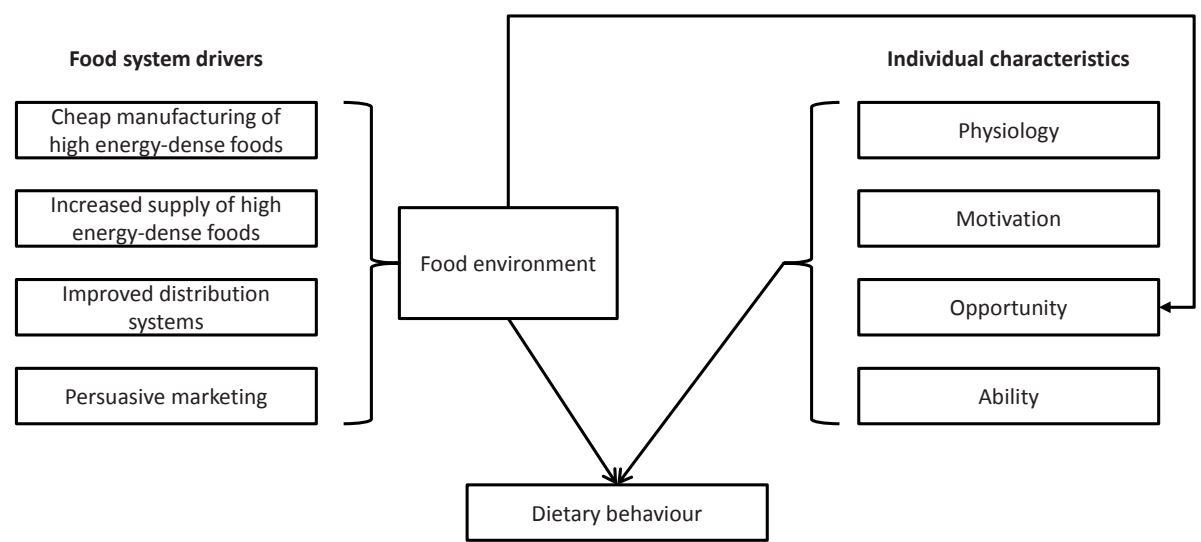

Figure 1.1: Food system drivers and individual characteristics that influence consumers' dietary behaviour

\section{Food system drivers}

For the food industry, manufacturing costs of high energy-dense foods have become relatively low during the $20^{\text {th }}$ and $21^{\text {st }}$ century, while retail value has stayed relatively 
high (Stuckler, McKee, Ebrahim, \& Basu, 2012). Particularly because of this costbenefit advantage over healthier products in terms of food production, food environments have been flooded with increasingly affordable unhealthy products, while healthy products remained relatively expensive (Monsivais \& Drewnowski, 2007; Swinburn et al., 2011). The same trend applies to the availability of unhealthy products; in supermarkets and points-of-purchase the proportion of high energydense, low-nutrient products has exceeded the proportion of nutritious low energydense products (Ni Mhurchu et al., 2013; Thornton et al., 2013). Moreover, the number of snack outlets that primarily sell high-calorie products has increased significantly (Burgoine et al., 2009; Pan, Malik, \& Hu, 2012).

In addition to increased availability and accessibility of affordable unhealthy foods, marketing of such products has contributed to the current obesity crisis (Seiders \& Petty, 2004). Particularly adolescents have proven to be susceptible to promotion of appealing, tasty and convenient unhealthy foods (Harris, Pomeranz, Lobstein, \& Brownell, 2009; Neumark-Sztainer, Story, Perry, \& Casey, 1999). The vast majority of the marketing budget of the food industry is spent on unhealthy products; as much as $89 \%$ percent of the television advertisements seen by adolescents concerns foods high in fat, sugar, and/or sodium (Powell, Szczypka, Chaloupka, \& Braunschweig, 2007).

Large-scale developments in the food system thus have provided consumers with an abundance of opportunities to consume excessive amounts of calories, which has been echoed by Social Ecological Models of behaviour (Sallis, Owen, \& Fisher, 2008). Additionally, individual characteristics influence the extent to which consumers struggle to adhere to healthy dietary behaviours within the current food environment.

\section{Individual characteristics}

As mentioned earlier, consumption of high energy-dense foods has been key for people's survival for centuries. This has led to genetic predispositions for these foods: children still have an innate preference for high energy-dense sweet foods (Ventura \& Mennella, 2011) while also adults prefer energy-dense foods that are high in fat, sugar, and salt (L. L. Birch, 1999).

In addition to environmental and evolutionary drivers, behavioural (change) models show that dietary behaviours are influenced by motivation- and ability-related barriers (Brug, 2008). While these theories differ in their operationalization of 
motivation and ability, for instance the Theory of Planned Behaviour names them attitudes and perceived behavioural control (Ajzen, 1991), while the Health Belief Model terms them level of perceived threat and self-efficacy (Rosenstock, Strecher, \& Becker, 1988), they all recognize motivation and ability related barriers as important drivers of dietary behaviours. Research employing these theories indeed shows that the presence of such barriers can lead to detrimental dietary behaviours (Baranowski, Cullen, Nicklas, Thompson, \& Baranowski, 2003; Conner, Norman, \& Bell, 2002).

Lock-in

The fact that consumers often choose unhealthy products because of a preference for fatty, sugary, and salty foods, and that food manufacturers continue to supply these products because of their profit margin, has led to a 'lock-in' within the food system in which the status quo is being maintained (Van Rijnsoever, Van Lente, \& Van Trijp, 2011). This lock-in has facilitated the continuing spread of the obesity epidemic. Intervention strategies that stimulate healthier dietary behaviour can breach this status quo within the food domain. The next sections discuss classifications, effectiveness and consumer evaluations of dietary interventions that have been documented in scientific literature.

\subsection{Classification of intervention strategies}

From a public health perspective, Michie et al. (2011) define interventions as "a coordinated set of activities designed to change specified behaviour patterns". This definition of interventions indicates a deliberate change of the current situation, the status quo. Therefore, in the present thesis, we define a food choice intervention as "a deliberate change in the current situation in the food domain, implemented by a stakeholder that has an interest in changing people's current food choice behaviour".

Within the food domain, intervention strategies come in many different shapes and forms, ranging from the use of labels on food packages to the elimination of unhealthy foods. Because of this breadth of intervention types, attempts to categorize them have resulted in several classification systems (Barry, Brescoll, Brownell, \& Schlesinger, 2009; Capacci et al., 2012; Malik, Willett, \& Hu, 2013; Nuffield, 2007; Rothschild, 1999; Traill et al., 2010). Classifications are important because by characterizing interventions, they enable improvement of the design and 
implementation of existing and future interventions (Michie et al., 2011). Additionally, classifications determine how intervention strategies are framed, both in the public policy debate and in scientific research. This intervention framing also influences the way people perceive a public health issue and how they react to attempts to address these issues (Shelley, 2012). For optimal communication between different stakeholders within the obesity problem, a comprehensive classification system of intervention strategies thus is required.

To be useful in terms of improving design and implementation of interventions, and thus to be of value for the present thesis, an intervention classification needs to meet three criteria (Michie et al., 2011): 1) the framework should be able to encompass all potential interventions (comprehensiveness), 2) the categories in the classification should be of the same specificity (coherence), and 3) the behaviour change mechanisms should be linked to theory (link to model of behaviour).

Classification frameworks ideally classify all interventions that have been or can be developed. A failure to do so may limit the scope of attempts to create novel interventions. In addition, the classification framework should ensure that the intervention categories are of the same type and specificity of entity. These criteria of comprehensiveness and coherence are met by (not exclusively) the Nuffield intervention ladder (Nuffield, 2007). The Nuffield ladder classifies interventions according to their effect on people's freedom of choice (i.e. intrusiveness). The ladder ranges from 'doing nothing' to 'eliminating choice', which it reaches after seven ordinal steps that become incrementally intrusive. Because of its focus on the effects of interventions on behavioural autonomy, the ladder is a widely used instrument in research on intervention evaluations (Diepeveen et al., 2013).

Furthermore, an understanding of the causes of the behaviour to be changed is needed to achieve behaviour change. With this understanding, intervention design can be improved by targeting specific intervention components to specific barriers for behaviour. In the food domain, behavioural theories that have been applied to dietary behaviours show that both individual-level and environmental determinants drive food-choice behaviour (Brug, 2008; Van Trijp, Brug, \& Van der Maas, 2005). Therefore, using an intervention classification framework that takes into account these different types of determinants is desirable. Rotschild's MotivationOpportunity-Ability (MOA) framework (1999) does so by stressing the importance of individual-level motivation (attitudes and personal norms towards the behaviour) and ability (skills and knowledge necessary for the behaviour), as well as 
environmental-level opportunity (physical and economic setting in which behaviour occurs) regarding the likelihood of healthy dietary behaviour. In addition, if motivation, opportunity, and/or ability is/are absent, the framework provides guidelines for the type of tools that should be used to intervene effectively: education (informing about behaviour to increase skills and knowledge and overcome ability-related barriers), marketing (accommodating behaviour by altering the physical and economic environment to overcome opportunity-related barriers), and/or law (coercing behaviour by restricting options to overcome motivationrelated barriers).

In conclusion, to meet the criteria for the classification of intervention strategies, the present dissertation uses the Nuffield intervention ladder to ensure comprehensiveness and coherence. Rothschild's MOA framework is used as the link to an overarching model of behaviour as well as a theoretical guide for tailoring of interventions to barriers for behaviour. Additionally, the Nuffield ladder is used to interpret how the tools from the MOA framework can be applied and how they influence people's freedom of choice (Figure 1.2).

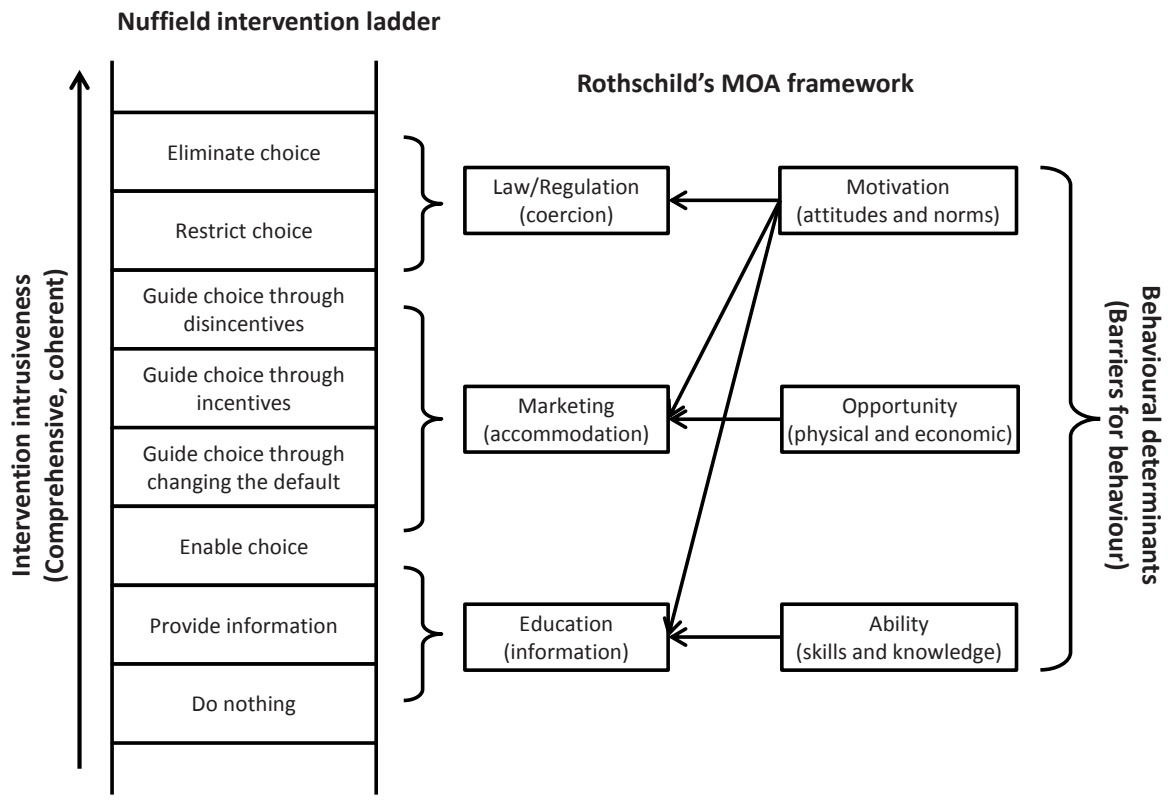

Figure 1.2: Overview of the connection between the Nuffield intervention ladder and Rothschild's MOA framework. 


\subsection{Effectiveness of intervention strategies for healthy dietary behaviours}

Although consensus exists that intervention strategies for stimulating healthy dietary behaviours play an indispensable role in the combat against overweight and obesity and associated diseases (Faulkner et al., 2011; Nestle \& Jacobson, 2000), the evidence for the behavioural impact of these strategies is inconclusive at best.

Reviews concerning the effectiveness of interventions for healthy dietary behaviours show that particularly the less intrusive strategies that focus on supporting informed choice have been largely ineffective in changing behaviour (Capacci et al., 2012; Mazzocchi et al., 2014). Such interventions aim to achieve behaviour change by providing people with the knowledge and skills to change that behaviour. Some studies indicate that interventions that aim to inform consumers only lead to very low increases in knowledge, thereby leaving behaviour unchanged (Elbel, 2011; Kocken, van Kesteren, Buijs, Snel, \& Dusseldorp, 2015). Other studies suggest that these interventions do succeed in increasing knowledge, but that this is rarely translated into behaviour change (Boles, Adams, Gredler, \& Manhas, 2014). Many scholars therefore conclude that providing people with information is a necessary but insufficient way to achieve behaviour change on a societal level (BrambilaMacias et al., 2011).

Reviews furthermore indicate that more intrusive intervention strategies that attempt to guide choices through incentives and disincentives are generally more effective than those that inform choice. Two types of (dis)incentives are distinguished: assortment (dis)incentives and monetary (dis)incentives. In the case of assortment (dis)incentives several studies show that both increasing availability of healthy choices and improving accessibility of healthy products can be effective ways to nudge consumers to healthier choices (Dayan \& Bar-Hillel, 2011; Rozin et al., 2011).

In the case of monetary incentives, research shows that lowering prices of healthy products can lead people to more often choose healthy options instead of unhealthy options (S. A. French et al., 2001; Powell, Chriqui, Khan, Wada, \& Chaloupka, 2013). A potential negative side effect of lowering the costs of obtaining healthy foods, however, is that people increase their consumption of these products and thereby actually increase their overall calorie intake (Epstein, Dearing, Roba, \& Finkelstein, 2010). 
In the case of monetary disincentives, studies on the effectiveness of taxes indicate that relatively low price increases on unhealthy products do not lead to significant changes in dietary behaviours (Chouinard, Davis, LaFrance, \& Perloff, 2007; Jacobson \& Brownell, 2000; Vallgarda, Holm, \& Jensen, 2015). Experimental research shows that price increases on unhealthy products only lead to healthier choices when they are substantial, around 25\% to 50\% (Giesen, Payne, Havermans, \& Jansen, 2011; Nederkoorn, Havermans, Giesen, \& Jansen, 2011). Such large price increases, however, are very unlikely to come into effect. Additionally, drastic price increases can lead people to choose cheaper versions of unhealthy products (substitution effect), which nullifies the desired effect of monetary interventions (Mytton, Clarke, \& Rayner, 2012).

The interventions that have proven to be most effective are those that are the most intrusive, such as restrictions or even eliminations of unhealthy choices. Studies regarding food choices made in vending machines and at check-out counters show that restricting unhealthy choices and replacing them with healthy options leads to increased sales of healthy products (Grech \& Allman-Farinelli, 2015; Van Kleef, Otten, \& Van Trijp, 2012). The issue with such intrusive interventions is that they potentially interfere with the free market situation and therefore the willingness to employ such strategies is low among policy makers (Greener, Douglas, \& Van Teijlingen, 2010). Additionally, consumers may perceive intrusive interventions to impede their freedom of choice, and therefore opt for alternatives, either in terms of products or points-of-purchase (Proudfoot \& Kay, 2014).

In conclusion, to date the effectiveness of different types of intervention strategies for healthy dietary behaviours has left much to be desired. Interest in both increasing effectiveness of existing interventions and developing new intervention strategies therefore has thrived recently (Traill et al., 2010). An important condition for maximizing effectiveness is gaining support for interventions from consumers, which will be discussed in the next section.

\subsection{Acceptance of intervention strategies}

How people evaluate intervention strategies can have significant consequences for the success of interventions, both in terms of the likelihood of implementation and effectiveness after implementation (Mazzocchi et al., 2014). Negative evaluations of 
(proposed) interventions potentially lead to organized resistance, which in turn could lead to non-implementation of interventions (Schaller, 2010; Vallgarda et al., 2015) and further deterioration of the problem that the interventions attempt to solve. Additionally, scholars stress that intervention evaluations influence how effective an intervention is and can be (Diepeveen, Ling, Suhrcke, Roland, \& Marteau, 2013; Gendall et al., 2015).

When confronted with an intervention, people either display reactance or rationalization (Laurin, Kay, \& Fitzsimons, 2012). Reactance is the process of valuing the status quo and responding negatively towards a (proposed) change, while rationalization refers to a positive response towards a change of the status quo by viewing this change in its most positive light (Kay, Jimenez, \& Jost, 2002). Which reaction will be evoked depends on both intervention- and individual characteristics.

\section{Effects of intervention characteristics on intervention acceptance}

Within the literature on evaluations of interventions that aim to change healthrelated behaviours, such as food choice behaviours, smoking, and alcohol consumption, acceptance of interventions (most often operationalized as the extent to which someone supports the implementation of an intervention) has emerged as one of the most relevant concepts (Diepeveen et al., 2013; Nuffield, 2007). Research shows that specific intervention characteristics influence people's acceptance of interventions. Particularly the magnitude of effect of an intervention on one's freedom of choice (i.e. intrusiveness) is an important predictor for the level of acceptance. Less intrusive interventions, such as information provision through labelling or public health campaigns, are generally more accepted than more intrusive interventions, such as price (dis)incentives and choice restriction (Pechey, Burge, Mentzakis, Suhrcke, \& Marteau, 2014). Additionally, the stage of implementation, also referred to as intervention absoluteness, influences acceptance: an intervention is generally more accepted when the likelihood of implementation is relatively certain, compared to when an intervention is unsure to come into effect (Laurin et al., 2012).

\section{Effects of individual characteristics on intervention acceptance}

In the food domain, research on consumer acceptance of intervention strategies furthermore focused on the influence of individual characteristics, in particular people's attributions of overweight and obesity (Hilbert, Rief, \& Braehler, 2007; Lusk \& Ellison, 2013; Pearl \& Lebowitz, 2014). The findings indicate that people who mainly attribute obesity to factors outside an individual's control accept intrusive 
measures more than people who mainly blame individuals themselves (Chambers \& Traill, 2011). Next to these studies acceptance of interventions in the food domain has received little attention, thereby leaving the potentially wide range of explanatory factors, related to both intervention- and individual characteristics, unexplored (Mazzocchi et al., 2014).

Transport policy research provides additional knowledge on the process of consumer acceptance of interventions (Eriksson, Garvill, \& Nordlund, 2008; Schuitema, Steg, \& Van Kruining, 2011; Steg, Dreijerink, \& Abrahamse, 2005; Steg \& Vlek, 2009). Studies in this application domain show that acceptance of interventions depends on two distinct drivers: general beliefs and intervention-specific beliefs. General beliefs concern people's beliefs about the magnitude of the problem that the intervention is trying to solve and the attribution of responsibility for the problem, which, as mentioned earlier, are also identified as important drivers in the food domain. Intervention-specific beliefs include people's perceptions of how effective and how fair interventions are; these have only sparsely been assessed in studies on intervention acceptance in the food domain.

Rothschild's Motivation-Opportunity-Ability (MOA) framework (1999) theorizes that effectiveness of interventions is maximized when they fit the barriers people have. It is plausible that also acceptance of interventions is influenced by the extent to which they fit people's specific barriers. People with type 2 diabetes and a low income (who therefore likely are motivated, but have less opportunity to choose healthy foods), for instance, likely accept interventions that decrease prices of healthy foods more than people who do have the opportunity but are not motivated to choose healthy foods (Marcy, Britton, \& Harrison, 2011).

Concluding, consumer acceptance of intervention strategies to change behaviour has been researched in different application domains and results show that acceptance can be driven by both intervention- and individual characteristics. For interventions in the food domain, however, the existence and the relative importance of these drivers are under-researched (Mazzocchi et al., 2014). Extending knowledge on the drivers of consumer acceptance of food choice interventions will provide policy makers with handles to maximize acceptance and overcome barriers regarding the implementation of interventions. 


\subsection{Scope and outline of the thesis}

Reviewing the literature on consumer evaluations of different types of intervention strategies, it can be concluded that, while consumer support for healthy food choice interventions is an important condition for long-term effectiveness, little is known about why consumers accept or do not accept these interventions. The overall aim of the present thesis therefore is to understand the process underlying acceptance of intervention strategies for healthy food choices. Specifically, we investigate the role that intervention characteristics (e.g. the source and the location of an intervention) and individual characteristics (i.e. consumers' perceived barriers for behaviour) play in this process.

To better understand the process of consumer acceptance of food choice interventions, the present dissertation aims to answer three research questions:

1. What are the drivers of consumer acceptance of intervention strategies for healthy food choices?

2. How do consumers' barriers for healthy food choices influence both food choices in real-life and acceptance of interventions?

3. How does intervention intrusiveness influence acceptance and effectiveness of intervention strategies for healthy food choices?

These three research questions will be answered in Chapters 2, 3, 4, and 5 . A proposed theoretical framework that also serves as a schematic outline of the chapters and their relation to each other is presented in Figure 1.3.

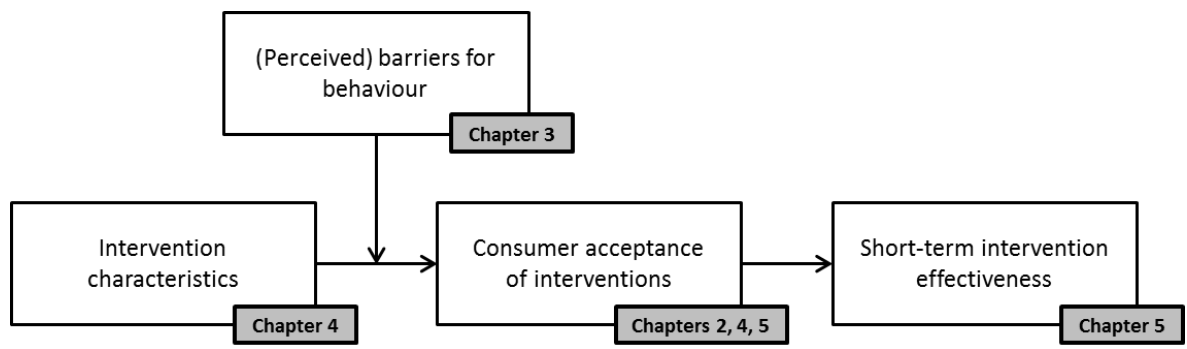

Figure 1.3: Schematic overview of the chapters 
To start uncovering the process of intervention acceptance in the food domain, the main drivers of healthy food choice interventions are identified. Chapter 2 reports about eight interviews and four focus groups in which the concept of acceptance in light of intervention strategies for healthy food choices is explored. Nine archetypical interventions that represent educational, marketing, and legal interventions, are used as reference points to elicit beliefs that are associated with acceptance. The chapter describes two main types of drivers of intervention acceptance that people bring forward when they are asked to explain why they do or do not accept intervention strategies: general beliefs and intervention-specific beliefs. Different underlying aspects of each driver are discussed and enriched with quotes from the interviews and focus groups.

To better understand (un)healthy food choice behaviour, the relation between people's perceived barriers for choosing healthy foods and their real-life food choices is examined. Chapter $\mathbf{3}$ presents an online survey, which assesses people's (perceived) barriers to choose healthy foods and self-reported food and beverage consumption. The chapter discusses the relation between barriers regarding the motivation, opportunity, and ability to choose healthy foods and beverages, and the habitual consumption of (un)healthy foods and beverages in real-life. Additionally, three dominant barrier profiles are identified and differences between these profiles regarding proportions of healthy choices, daily meal consumption, and (socio)demographic characteristics are discussed.

To further uncover the process underlying acceptance, both the effect of intervention intrusiveness on acceptance and the role that the perceived effectiveness and fairness play in this relation are examined. Chapter 4 presents an additional wave of data collection of the online survey, in which people evaluate 16 hypothetical interventions strategies with differing levels of intrusiveness on the constructs of effectiveness (personal- and societal effectiveness), fairness, and acceptability. For generalization purposes, the interventions furthermore differ on the following characteristics: source, location, approach/avoidance, type, and severity. Differences in evaluations of interventions between the dominant barrier profiles (identified in Chapter 3) are discussed and a conceptual model for the process of intervention acceptance in the food domain is provided.

To investigate whether the process of acceptance, as identified in Chapter 4, generalizes to different stages of intervention implementation (absoluteness), evaluations of interventions that are implemented in a realistic setting are 
investigated. Chapter 5 reports about an experiment in which people choose a snack food and a beverage from a vending machine. In addition to a control condition without an intervention, four interventions are implemented in the vending machine (ranging from low to high intrusiveness): provision of information, assortment incentives, price disincentives, and restriction of choice. After choosing two products, people rate the intervention that was implemented in the vending machine on perceived effectiveness, perceived fairness, and acceptability. Subsequently, the three interventions of the other conditions (which people did not encounter) are evaluated on paper. The chapter discusses differences regarding intervention evaluations, actual food choices (short-term effectiveness), and food choice satisfaction between the four intervention types, as well as differences between evaluations of realistic and hypothetical intervention settings within the intervention types.

Chapter 6 concludes the present thesis by providing a general discussion of the results that are described in Chapters 2, 3, 4, and 5. In addition, recommendations for future research and limitations of the studies will be analysed. 



\section{UNDERSTANDING CONSUMER ACCEPTANCE OF INTERVENTION STRATEGIES FOR HEALTHY FOOD CHOICES}

A qualitative study

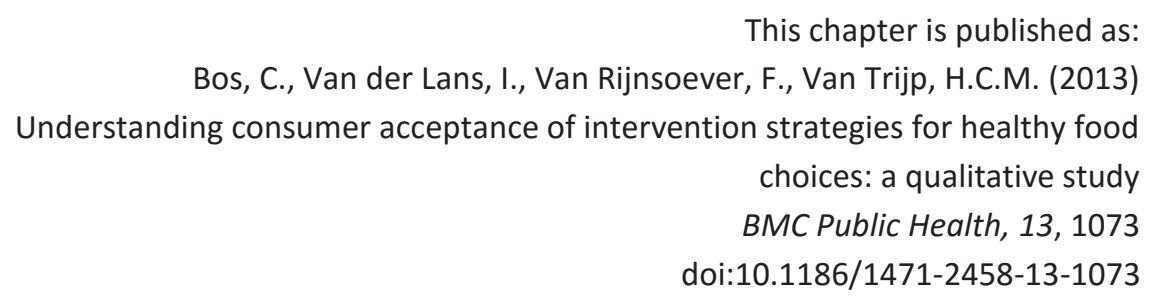




\section{Abstract}

\section{Background}

The increasing prevalence of overweight and obesity poses a major threat to public health. Intervention strategies for healthy food choices potentially reduce obesity rates. Reviews of the effectiveness of interventions, however, show mixed results. To maximize effectiveness, interventions need to be accepted by consumers. The aim of the present study is to explore consumer acceptance of intervention strategies for low-calorie food choices. Beliefs that are associated with consumer acceptance are identified.

\section{Methods}

Data was collected in the Netherlands in 8 semi-structured interviews and 4 focus group discussions $(\mathrm{N}=39$ ). Nine archetypical strategies representing educational, marketing, and legal interventions served as reference points. Verbatim transcriptions were coded both inductively and deductively with the framework approach.

\section{Results}

We found that three beliefs are related to consumer acceptance: 1) general beliefs regarding obesity, such as who is responsible for food choice; 2) the perceived effectiveness of interventions; and 3 ) the perceived fairness of interventions. Furthermore, the different aspects underlying these general and interventionspecific beliefs were identified.

\section{Conclusions}

General and intervention-specific beliefs are associated with consumer acceptance of interventions for low-calorie food choices. Policymakers in the food domain can use the findings to negotiate the development of interventions and to assess the feasibility of interventions. With respect to future research, we recommend that segments of consumers based on perceptions of intervention strategies are identified. 


\subsection{Background}

From 1980 to 2008, the overweight and obese population almost doubled worldwide. It now consists of an estimated 1.46 billion adults and 170 million children (Swinburn et al., 2011). People with overweight and obesity are more vulnerable to non-communicable diseases such as type II diabetes, cancer, and cardiovascular diseases (Guh et al., 2009). The costs of these diseases in terms of quality of life and healthcare are enormous. The medical expenditure associated with obese individuals is estimated at 30 per cent higher than normal-weight peers (Withrow \& Alter, 2011). Thus, overweight and obesity pose both personal and public health concerns.

The growth of obesity rates is the result of a systematic energy imbalance primarily due to excessive intake of calories (Bleich, Cutler, Murray, \& Adams, 2008). Although (the lack of) physical activity is an important part of the obesity problem, curtailment of overconsumption is of greater importance. Limiting calorie intake directly affects weight status and also adds to the impact of physical activity on weight status (Cohen, Sturm, Scott, Farley, \& Bluthenthal, 2010). Intervention strategies that reduce the amount of calories consumers choose potentially achieve both economic benefits and improvements of personal and public health (Brownell et al., 2009).

A broad array of intervention strategies, varying from public health campaigns to the taxation of high-calorie foods, has been implemented to reduce obesity prevalence (Capacci et al., 2012). However, recent systematic reviews concerning the effectiveness of intervention strategies show mixed results. A large structured analysis of policy interventions (Capacci et al., 2012) reveals inconclusive results about the behavioural impact of different types of interventions. Other reviews also claim that for many interventions only limited evidence of their effectiveness can be found (Brambila-Macias et al., 2011; Kesten, Griffiths, \& Cameron, 2011; Maes et al., 2012; Paul-Ebhohimhen \& Avenell, 2008).

To increase the effectiveness of interventions, Andreasen (2002) argues that they need to be consumer-driven. More specifically, intervention strategies should be based on an understanding of consumers' experiences, values, and needs, which jointly accumulate into consumer acceptance of intervention strategies. The term 'consumer acceptance' is used throughout the article because the interventions of interest target food choice (consumption) behaviour. It is important to note that the 
present study's method allowed for assessing these interventions from a consumer point of view as well as from a broader, social perspective.

The degree of consumer acceptance affects both the effectiveness and the implementability of interventions. Reactance theory (Brehm, 1966) suggests that low levels of acceptance towards an intervention cause consumers to adopt or strengthen an attitude that is contrary to the desired behaviour, thereby increasing resistance to perform the desired behaviour. In contrast, a high level of acceptance elicits rationalization, causing consumers to be more likely to approve of interventions and to adopt the intended behaviour (Laurin et al., 2012). In addition, the acceptability of an intervention is an important condition for its implementation. Stakeholders will be reluctant to intervene without public support (Schuitema et al., 2011). Knowledge of factors influencing consumer acceptance of intervention strategies thus is crucial. Within the food domain, a number of surveys have found that beliefs about the causes of obesity affect support for obesity prevention policies (Barry et al., 2009; Hilbert et al., 2007; Oliver \& Lee, 2005). However, a structured insight into consumers' perceptions of interventions is lacking (Traill et al., 2010).

The present study aims to fill this gap by exploring not only consumer acceptance, but also the perceived effectiveness and the perceived fairness of intervention strategies for low-calorie food choices. A qualitative approach is adopted to identify concepts and processes at the individual level that will enrich the dominant quantitative focus on the effectiveness of interventions. The present study uses both social marketing theory and existing research on consumer acceptance of interventions as a theoretical framework. The results provide guidance for the assessment of consumer acceptance of (future) interventions in the food domain.

\subsection{Theoretical framework}

\section{Social marketing}

Social marketing uses commercial marketing concepts such as market segmentation and consumer research to achieve social change (Van Trijp, 2010). A growing body of research claims that social marketing provides a promising framework for improving health both at the individual level and the wider societal level (Gordon, McDermott, Stead, \& Angus, 2006). A significant part of social marketing is to understand consumers before and at the outset of interventions (Andreasen, 2002). 
To achieve this understanding for intervention strategies in the food domain, learnings are extracted from the environmental domain (e.g. car use vs. public transport), where acceptance of interventions has been studied more extensively (Eriksson et al., 2008; Gärling \& Loukopoulos, 2007; Steg et al., 2005). Within that domain, general beliefs and intervention-specific beliefs have proven to affect acceptance of interventions. General beliefs reflect one's overall orientations, opinions and attitudes towards a particular public issue. An example of a general belief in the environmental domain is one's perception of responsibility for traffic congestion. In addition, two intervention-specific beliefs emerge from that body of literature: the perceived effectiveness and the perceived fairness of interventions (Eriksson et al., 2008). The perceived effectiveness refers to whether an individual believes that an intervention will actually lead to the intended behaviour; therefore it does not necessarily reflect an intervention's actual effectiveness. Likewise, the perceived fairness relates to an individual's belief that the implementation of a specific intervention is a fair way to stimulate the intended behaviour. Our research framework (Figure 2.1) serves to identify both the general and intervention-specific beliefs for the food domain. 


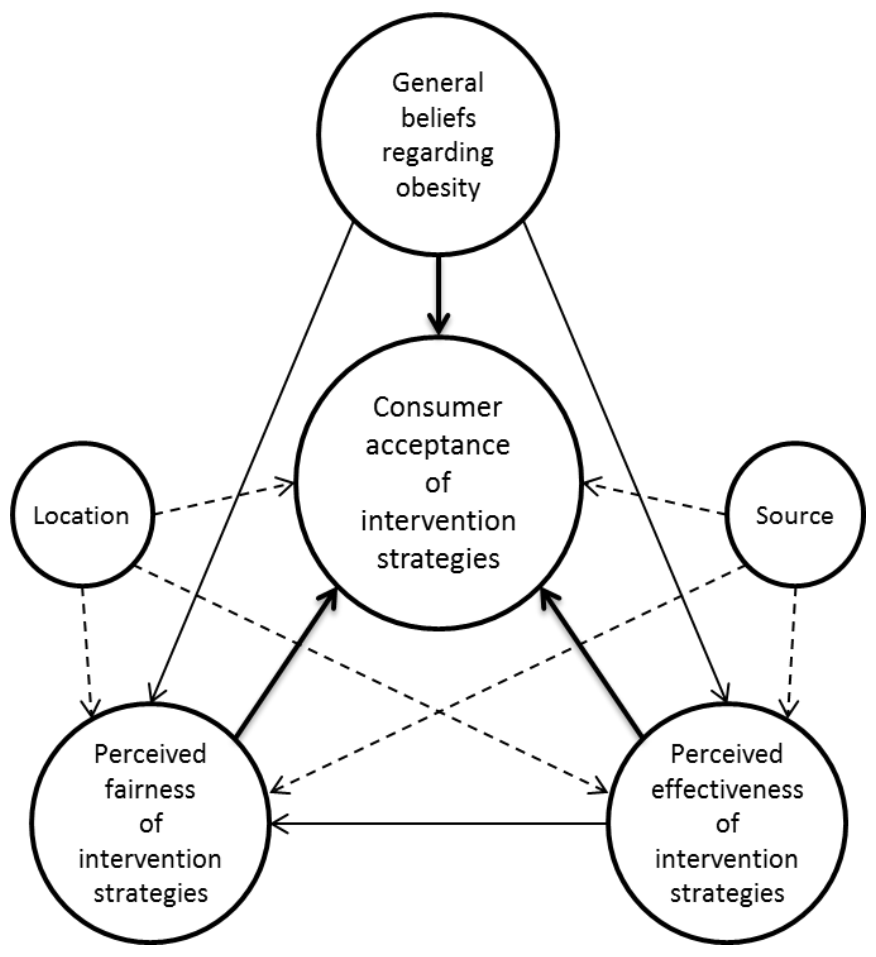

Figure 2.1: Schematic representation of the research framework.

Continuous lines: relations of interest that are found in the environmental domain.

Dotted lines: additional relations of interest.

\section{Types of intervention strategies}

Various classifications of intervention strategies for public health issues exist (Capacci et al., 2012; Nayga, 2008; Rothschild, 1999; Van Rijnsoever et al., 2011). Although these classifications share similarities, most of them are strongly policyoriented. Among them, Rothschild's social marketing framework allows for the exploration of both policy and non-policy oriented intervention strategies.

Rothschild distinguishes between three types of tools for intervention strategies in public health issues: education, marketing and law. These tools differ on the basis of their reinforcement/reward and degree of voluntary change. Education refers to voluntary adaptation of behaviour by providing information to consumers. Marketing also refers to voluntary adaptation of behaviour; however it does so by reinforcing consumers. Law refers to non-voluntary adaptation of behaviour by using 
coercion and by punishing consumers for non-compliance. The present study uses nine archetypical interventions based on these three tools. The archetypes and matching examples are adapted from Van Trijp et al. (2005) and can be found in Table 2.1.

Table 2.1: List of archetypical intervention strategies and corresponding examples Interventions

\begin{tabular}{|c|c|}
\hline Interventions & Example \\
\hline & Law \\
\hline $\begin{array}{l}\text { Making unhealthier products more } \\
\text { expensive }\end{array}$ & An increase of taxes on high-calorie products \\
\hline $\begin{array}{l}\text { Making healthier products less } \\
\text { expensive }\end{array}$ & A decrease of taxes on low-calorie products \\
\hline $\begin{array}{l}\text { Restricting the promotion of } \\
\text { unhealthier products }\end{array}$ & $\begin{array}{l}\text { Prohibition of promotion of high-calorie products at bus } \\
\text { shelters } \\
\text { Marketing }\end{array}$ \\
\hline Promoting healthier products & $\begin{array}{l}\text { Promotion of a low-calorie product by a famous athlete } \\
\text { on behalf of the food supplier }\end{array}$ \\
\hline $\begin{array}{l}\text { Decreasing the accessibility of } \\
\text { unhealthier products } \\
\text { Increasing the availability of } \\
\text { healthier products }\end{array}$ & $\begin{array}{l}\text { Placement of high-calorie products on the bottom shelf } \\
\text { and low-calorie products at eyesight in a supermarket } \\
\text { Provision of low-calorie alternatives for high-calorie } \\
\text { products by food suppliers } \\
\text { Education }\end{array}$ \\
\hline $\begin{array}{l}\text { Providing calorie information of } \\
\text { personal choices in relation to } \\
\text { choices of others }\end{array}$ & $\begin{array}{l}\text { Use of a receipt that indicates the amount of calories } \\
\text { one has bought and the amount others buy, } \\
\text { implemented in a canteen by the employer }\end{array}$ \\
\hline $\begin{array}{l}\text { Providing food labels with calorie } \\
\text { information }\end{array}$ & $\begin{array}{l}\text { Provision of extensive traffic-light labels on food } \\
\text { products by food suppliers }\end{array}$ \\
\hline $\begin{array}{l}\text { Providing information about } \\
\text { healthier eating habits }\end{array}$ & $\begin{array}{l}\text { Provision of information about how to create low- } \\
\text { calorie eating habits through a governmental campaign }\end{array}$ \\
\hline
\end{tabular}

In addition to distinguishing between education, marketing, and law, our framework also explores the effects of both the intervention's physical location (e.g. restaurants and schools) and the intervention's source/manager (e.g. the government and the food industry) of an intervention on consumer acceptance. Among the spectrum of interventions, consumer acceptance likely differs due to the different implications that combinations of tools, locations, and sources of interventions have for consumers (Ten Have et al., 2011). 


\subsection{Methods}

\section{Ethical approval}

The present study's interview and focus group protocol was submitted to the Social Science Ethics Committee of the Wageningen University and subsequently approved for fulfilling the Wageningen University code of conduct.

\section{Design and participants}

Data was collected in the Netherlands in two rounds. The first round consisted of eight semi-structured interviews with an average duration of 50 minutes and the second round used four focus group discussions (6-9 participants), each 2 hours long. The interviews gained individual views on the concept of acceptability, while the focus groups captured the dynamics and the range of the concept of acceptability by allowing participants to build on and react to responses of others (Stewart \& Shamdasani, 1990). The interviews also provided the possibility to make procedural adjustments for the focus groups when needed.

Participants were recruited through a recruitment agency, which uses its own panel. Selection of participants aimed at a sufficiently heterogeneous sample in terms of gender, age and income level (Table 2.2). A written informed consent was obtained from participants before the start of the interviews and focus groups. For their participation, participants received the standard monetary compensation, according to the recruitment agency's policy. Both the interviews and the focus groups were held at the recruitment agency's facilities and were conducted by the first author. During the focus groups, an observer was present to take notes and to ensure all items in the guide were addressed. 
Table 2.2: Sample characteristics

\begin{tabular}{lcc}
\hline & $\begin{array}{c}\text { Interviews Focus groups } \\
(\mathbf{n}=\mathbf{8})\end{array}$ & \begin{tabular}{l}
$(\mathbf{n}=\mathbf{3 1})$ \\
\hline Gender
\end{tabular} \\
Male & 5 & 14 \\
Female & 3 & 17 \\
Age & & \\
18-35 & 3 & 13 \\
$35-50$ & 3 & 10 \\
50-65 & 2 & 8 \\
Income level & & \\
Less than modal & 3 & 10 \\
Approximately modal & 3 & 14 \\
Higher than modal & 2 & 7 \\
\hline
\end{tabular}

\section{Stimuli and materials}

The stimulus material consisted of eight archetypical intervention strategies (Table 1). Each intervention was presented on a separate A5 size paper following a standardised format with three blocks of information. The first block gave a definition of the archetypical intervention, e.g. "Making unhealthier product more expensive". The second block provided a detailed example of the archetype that more firmly categorised the interventions in terms of education, marketing, and law: "An increase of taxes on high-calorie products". The third block was used to provide an image of the detailed example.

In addition to the eight interventions used in the interviews, one extra intervention was introduced during the focus groups. Based on remarks during the interviews, "Restricting the promotion of unhealthier products" was added as a ninth intervention. Half of the interview participants expressed the opinion that excessive promotion of high-calorie foods was a significant contributor to the obesity problem. Some therefore suggested adding an intervention that restricted such promotion to the other archetypical interventions.

\section{Procedure}

The interviews were divided into five parts (Table 2.3). After a short introduction, the interviewer explicitly invited and encouraged participants to voice their personal opinions and stressed that there were no correct or wrong answers. As a warm-up, participants were asked questions about their interpretation of (un)healthy food choices and the perceived responsibility for food choices. Following the warm-up, 
the interviewer explained that in the context of the interview, the interpretation of a healthy choice was restricted to a choice relatively low in calories, whereas an unhealthy choice was restricted to a choice with a relatively high caloric value. This interpretation was used to avoid that participants would define healthy choices at different levels of abstraction (Ronteltap, Sijtsema, Dagevos, \& de Winter, 2012).

Table 2.3: Interview and focus group topics

\begin{tabular}{|c|c|}
\hline Topic & Content \\
\hline Part 1: Introduction & Consent form and word of welcome \\
\hline \multirow[t]{2}{*}{ Healthy and unhealthy choices } & What, in your opinion, is a(n) (un)healthy food choice? \\
\hline & Do you find it easy or hard to make healthy food choices? \\
\hline Responsibility for food choices & $\begin{array}{l}\text { Who is responsible for the healthiness of the food choices } \\
\text { you make? }\end{array}$ \\
\hline $\begin{array}{l}\text { Part 2: Acceptability of } \\
\text { intervention strategies }\end{array}$ & $\begin{array}{l}\text { Do you think changes are warranted to stimulate low-calorie } \\
\text { food choices? }\end{array}$ \\
\hline $\begin{array}{l}\text { Introduction of the } \\
\text { archetypical intervention } \\
\text { strategies }\end{array}$ & $\begin{array}{l}\text { Do you think the archetypical interventions are acceptable, } \\
\text { when their goal is to stimulate you to make low-calorie food } \\
\text { choices? }\end{array}$ \\
\hline $\begin{array}{l}\text { Part 3: Perceived effectiveness } \\
\text { of intervention strategies }\end{array}$ & $\begin{array}{l}\text { Do you think the archetypical interventions will lead you to } \\
\text { make low-calorie food choices? }\end{array}$ \\
\hline $\begin{array}{l}\text { Part 4: Perceived fairness of } \\
\text { intervention strategies }\end{array}$ & $\begin{array}{l}\text { Do you think the archetypical interventions are a fair way to } \\
\text { stimulate you to make low-calorie food choices? }\end{array}$ \\
\hline $\begin{array}{l}\text { Part 5: Acceptability of } \\
\text { intervention strategies (2) }\end{array}$ & $\begin{array}{l}\text { Do you think the archetypical interventions are acceptable, } \\
\text { when their goal is to stimulate you to make low-calorie food } \\
\text { choices? }\end{array}$ \\
\hline Wrap-up & Explanation of the research context and a word of thanks \\
\hline
\end{tabular}

To explore participants' initial attitude towards interventions, the interviewer asked them whether they thought changes were needed to stimulate healthy food choices. Subsequently, the interviewer introduced the archetypical interventions one by one and in a fixed order. Next, participants were asked the interview's main question: "Do you think these interventions are acceptable, when their goal is to stimulate you to make low-calorie food choices?" The interviewer instructed participants to individually sort the interventions into three groups: Acceptable, Neutral/don't know, and Not acceptable. After the sorting task, the interviewer asked participants to elaborate on their classification. The interviewer then asked if and how each archetypical intervention could become more and/or less acceptable. Through the use of prompts participants were also asked whether the source and the location of an intervention influenced its acceptability (e.g. "Do you think this intervention would be more/less acceptable if it was executed by another source?" and "Do you 
think this intervention would be more/less acceptable at other locations?"). Last, the interviewer asked participants whether they thought the interventions were acceptable for others and/or certain groups. Part 2 took up about half of the time of both the interviews ( \pm 25 minutes) and the focus groups ( \pm 55 minutes).

After a 5-minute break, the procedure for Part 2 was repeated for the perceived effectiveness (Part 3) and the perceived fairness (Part 4) of the archetypical interventions. For these parts the main questions were "Do you think the archetypical interventions will lead you to make low-calorie food choices?" and "Do you think the archetypical interventions are a fair way to stimulate you to make lowcalorie food choices?", respectively. However, upon completion of the individual sorting task, a smaller number of interventions were addressed during these parts of the discussion due to time constraints. The moderator did ensure that all tools (education, marketing, and law) were covered.

During Part 5 participants were instructed once more to sort the interventions on the basis of their acceptability, without the opportunity to refer to the initial classification of Part 2. Differences between the classifications in Part 2 and Part 5 were then identified and participants were given the opportunity to elaborate on any changes they made. Last, the interviewer explained the context of the study and participants were given the opportunity to remark on the interview. A word of thanks and the distribution of a monetary compensation concluded each session.

Because no methodological issues emerged during the interviews, the same procedure was used for the focus group discussions. The moderator also used the same questions and stimuli materials for the discussions (except for one additional intervention scenario).

A noteworthy finding is that during the opportunity to give remarks, participants more than once declared that they were pleased to have been part of such a discussion. They particularly appreciated the opportunity to voice their own opinions and to discuss public health issues with each other. Therefore, there is little reason to believe that social desirability influenced the legitimacy of participants' responses.

\section{Data analysis}

The semi-structured interviews and focus group discussions were recorded and transcribed verbatim by the first author and two assistants. Atlas.ti 6.2 (Atlas.ti Scientific Software Development $\mathrm{GmbH}$, Berlin, Germany) was used to carry out the 
content analysis. To analyse the data, the framework approach was used (Pope, Ziebland, \& Mays, 2000). This means that after familiarisation with the data by listening to the audio, watching video footage and reading the transcripts, dominant themes were extracted both deductively and inductively. The deductive part consisted of identifying quotes and aspects that fitted the beliefs of the research framework. The inductive part of the coding consisted of identifying additional themes and aspects that emerged from the data itself.

The interview transcripts were analysed and coded first, resulting in an initial codebook. Subsequently, this codebook was used to analyse the focus group transcripts while leaving room for new codes to emerge. During the analysis of the last focus group transcript no new codes emerged, suggesting that theoretical saturation was reached.

\subsection{Results}

The results are discussed around the three beliefs that were part of the research framework: general beliefs regarding obesity, the perceived effectiveness of interventions, and the perceived fairness of interventions. Table 2.4 displays the three beliefs and the underlying aspects that emerged. The table also gives an overview of the amount of quotes that were given about these aspects during the different parts of the interviews and focus groups. If applicable, the results section distinguishes between education, marketing, and legal interventions. 
Table 2.4: Number of quotes given about aspects of the general and interventionspecific beliefs in the eight interviews (I) and the four focus groups (FG)

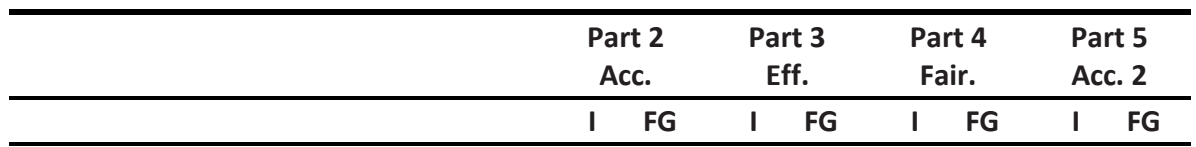

1. General beliefs regarding obesity

$\begin{array}{lrrrrrrrr}1.1 \text { Responsibility for food choice } & 7 & 9 & 4 & 4 & 0 & 11 & 3 & 6 \\ 1.2 \text { Problem awareness } & 8 & 14 & 0 & 2 & 3 & 10 & 1 & 2\end{array}$

2. Perceived effectiveness of interventions

\begin{tabular}{lrrrrrrrr} 
2.1 Perceived personal effectiveness \& & 8 & 14 & 0 & 2 & 3 & 10 & 1 & 2 \\
Perceived societal effectiveness & 13 & 13 & 25 & 21 & 7 & 9 & 0 & 0 \\
2.2 Effectiveness other domains & 6 & 5 & 8 & 4 & 3 & 5 & 1 & 1 \\
2.3 Accessibility of low-calorie products & 7 & 10 & 2 & 5 & 1 & 9 & 0 & 0 \\
2.4 Healthiness of low-calorie products & 4 & 13 & 2 & 8 & 4 & 5 & 0 & 0 \\
2.5 Identifiability low-calorie products & 8 & 12 & 4 & 19 & 5 & 2 & 0 & 0 \\
2.6 Combinations of interventions & 7 & 10 & 4 & 3 & 4 & 2 & 0 & 1 \\
3. Perceived fairness of interventions & & & & & & & & \\
3.1 Encouragement vs. discouragement & 11 & 11 & 0 & 3 & 5 & 11 & 0 & 0 \\
3.2 Societal fairness & 5 & 12 & 0 & 2 & 4 & 6 & 0 & 3 \\
3.3 Effects on consumer groups & 5 & 14 & 4 & 3 & 2 & 2 & 2 & 2 \\
3.4 Effects on food industry & 3 & 8 & 2 & 2 & 1 & 2 & 1 & 0 \\
3.5 Fairness of disseminated information & 24 & 23 & 5 & 17 & 5 & 4 & 0 & 5 \\
3.6 Liberty, autonomy, and privacy & 5 & 6 & 1 & 8 & 11 & 23 & 4 & 2 \\
Source & 8 & 16 & 8 & 11 & 5 & 5 & 0 & 2 \\
Location & 22 & 13 & 6 & 10 & 5 & 14 & 0 & 5 \\
\hline
\end{tabular}

For illustration purposes, several quotes are given. These quotes, which characterise the different beliefs and aspects, come from different parts of the interviews and discussions (for example: a quote regarding the effectiveness of an intervention could have been given during a discussion about the acceptability of that intervention). Furthermore, results from the interviews and the focus groups are discussed jointly because no differences existed with respect to the beliefs and underlying aspects that emerged.

Consumer acceptance of intervention strategies for low-calorie food choices Figure 2.2a depicts the acceptability of the nine archetypical interventions. When participants were asked to elaborate on the acceptability of interventions in Part 2, beliefs about both the effectiveness and the fairness of interventions frequently 
came up. Because these intervention-specific beliefs were also addressed individually in Part 3 and Part 4, respectively, they will be discussed separately in further parts of the results section.

In addition to intervention-specific beliefs, participants also often expressed general beliefs regarding the obesity problem while elaborating on the acceptability of interventions. The next section gives an overview of the themes that can be discerned.

\section{General beliefs regarding obesity}

Two dominant themes emerged with respect to general beliefs regarding obesity: responsibility for food choice and problem awareness.

\section{Responsibility for food choice}

A first theme that emerged was the responsibility for food choice. Some participants expressed that, above all, an individual is personally responsible for food choice. Therefore they expressed less acceptance of being stimulated to choose low-calorie foods through interventions:

"I think I'm responsible for what I buy. (...) What gets in my house and what I eat, as a consumer I'm responsible for that. Who is someone else to tell me what to choose?"

Although virtually all participants acknowledged consumers' own responsibility for making food choices, a large number also pointed at the additional responsibility of other stakeholders such as the government and food suppliers. Many of these participants expressed higher acceptance of interventions, especially strategies based on education and the marketing of low-calorie food products. As one man stated: 

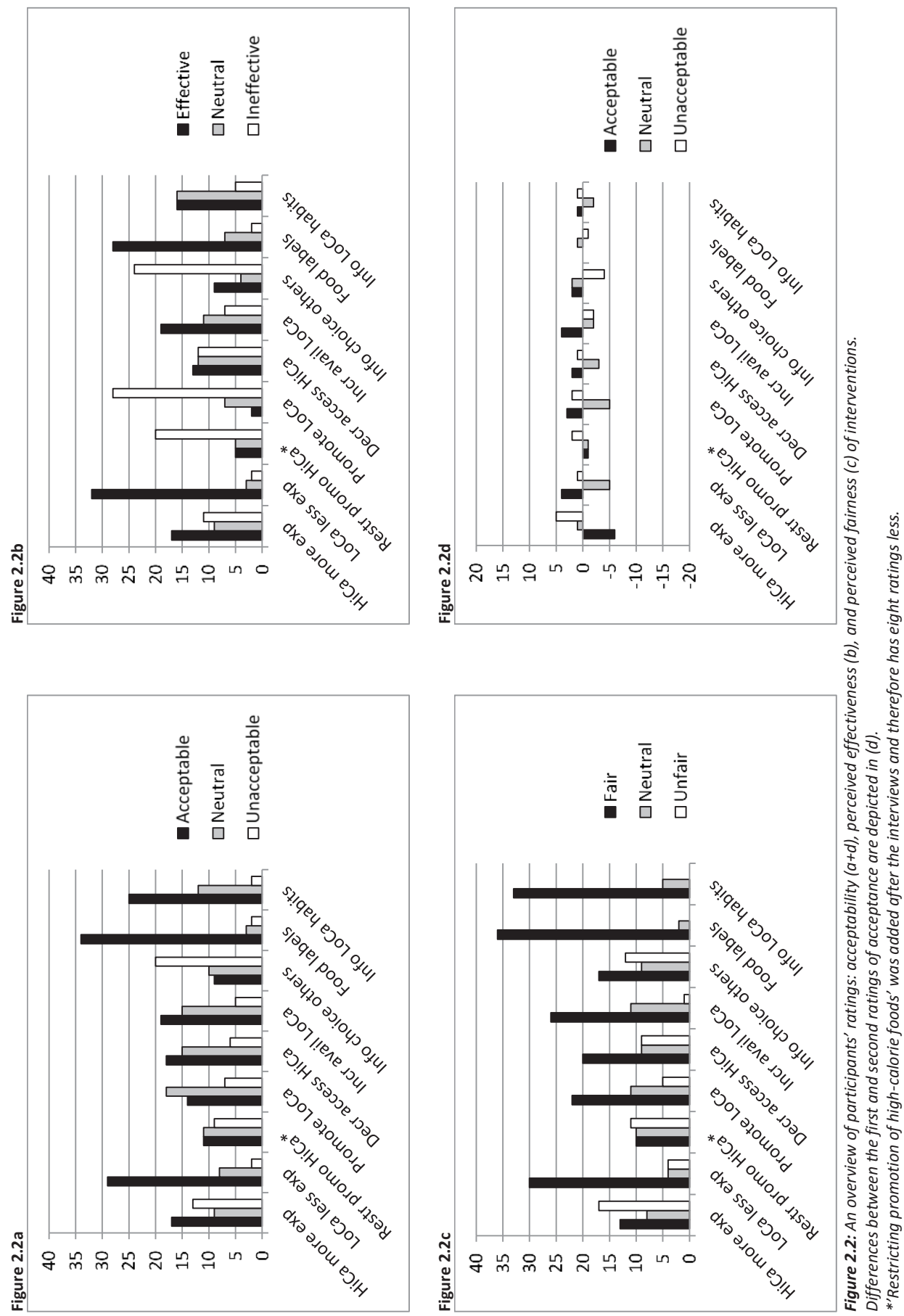
"I think that marketing of food suppliers certainly has a big influence (on consumer choice). The way they approach consumers; I think they do have a responsibility to help consumers choose healthily".

Participants also frequently mentioned parents' responsibility for the food choices of their children. All agreed that parents are fully responsible for the healthiness of their children's food choices and therefore mentioned mothers and fathers as most acceptable sources for interventions. Within the scope of parental interventions, both education about the healthiness of foods and the marketing of healthy choices at home were perceived as most acceptable.

\section{Problem awareness}

In addition to responsibility for food choice, problem awareness emerged as a second general belief that was related to the acceptance of interventions. Participants recognised that obesity rates have risen during the last decades. Most of the participants attributed this increase to a food environment that promotes overeating. The main characteristics of such an environment consisted of both the availability of high-calorie fast-foods and the relative price advantage of high-calorie foods over low-calorie foods. A majority of participants felt that interventions generally were acceptable if they improved the healthfulness of the environment. A minority disagreed, however, and expressed less acceptance of interventions by claiming that the current food environment provides enough opportunity to maintain a healthy lifestyle.

Another issue that contributed to the obesity problem was the indistinctness of healthy and unhealthy choices. Participants regularly expressed dissatisfaction with opposing claims regarding food products in the media. The resulting ambiguity impaired the ability to make healthy choices. As an older man put it:

"In the 70's they (the government) promoted certain fats. But they stopped doing that because it wasn't healthy. Currently, it's the same with milk: some say it's good for you, other say it's not. How do I know what is true?"

\section{Perceived effectiveness of intervention strategies for low-calorie food choices} The upcoming section will describe the aspects that emerged with regard to the perceived effectiveness of interventions. Ratings of the perceived effectiveness of the archetypical interventions are depicted in Figure 2.2b. 


\section{Perceived personal and societal effectiveness of interventions}

Participants often distinguished between how effective they perceived interventions to be for themselves and for society as a whole. While some participants thought that the effectiveness of interventions was fairly equal on a personal and societal level, others did not.

Participants who did perceive differences in effectiveness particularly pointed at educational and marketing interventions. They stated that these interventions would not be effective for them personally, but they would be on a societal level. A woman claimed:

"For me this (traffic-light labelling) would not lead to more low-calorie choices. But I do think that others who are not aware of nutritional values would choose lowcalorie more often because of these labels."

\section{Perceived effectiveness of similar interventions in other domains}

To assess the effectiveness of interventions for low-calorie food choices, participants often relied on their personal knowledge about the effectiveness of tobacco-, alcohol- and transport interventions. They used this knowledge to infer the effectiveness of similar interventions in the food domain. Referring to the domain of tobacco, a majority of participants considered marketing interventions that decrease the accessibility of high-calorie foods to be effective:

"You can eat unhealthy everywhere currently, just like you could smoke everywhere in the past. Nowadays that's different; there's an immense pressure on smoking and for that reason I see less people smoke. That could happen with unhealthy foods as well".

Using a similar analogy, a few participants claimed that educational interventions are ineffective by pointing at a lack of success in other domains. Referring to on-pack nutrition labels, a current smoker stated:

"It's like with cigarettes, the box contains warnings as 'Smoking is unhealthy' and 'Smoking kills', but everyone continues smoking".

\section{Accessibility of low-calorie products}

Participants furthermore stressed that interventions need to make low-calorie choices accessible both physically and financially to be effective. Some participants 
reported that they knowingly make fewer healthy choices because of the relatively high price of such choices. To increase effectiveness, marketing and legal interventions should therefore bridge the gap between prices of cheap high-calorie foods and more expensive low-calorie foods. As a female student explained:

"As a student I regularly choose French fries, because they only cost €1.50. If I want to eat a healthy sandwich, that will cost me almost three times as much! If it was equally expensive, I would choose more healthily."

Similar worries were expressed concerning the physical accessibility of low-calorie food choices. In line with the aforementioned food environment that promotes overeating, participants thought that high-calorie choices were more accessible than low-calorie choices. A majority therefore stated that interventions should make lowcalorie choices easier to obtain physically. Some participants saw an opportunity for the food supply to provide (new) low-calorie versions of high-calorie choices. As one young man stated:

"If I made the choice to eat a pizza, I will not all of the sudden choose a salad. But if there would be two choices, a normal and a low-calorie version, then I would choose the latter".

Participants recommended school canteens as locations where marketing strategies that increase accessibility of low-calorie foods would be most effective. A majority perceived a shortage of those products in the assortment at school canteens currently. In contrast, participants contested the effectiveness of increasing accessibility of low-calorie choices in restaurants. The main argumentation for this finding concerned participants' goal of eating out. Almost all described its purpose along the lines of 'being away' and 'having fun', thereby indicating that the caloric value of food choices was of less importance.

\section{Perceived healthiness of low-calorie choices}

Related to the importance of accessibility, participants stressed that the perceived healthiness of low-calorie choices plays a crucial role in the effectiveness of interventions as well. Some reported to have doubts regarding the healthiness of low-calorie choices. While all participants saw fruit and vegetables as healthy lowcalorie choices, some were sceptical of the healthiness of other low-calorie products. Frequently mentioned products that induced this scepsis were light versions of soft drinks and pizzas. Participants perceived the additives and the ingredients that 
replace the sugar and the fat in light products to be unhealthy, and therefore less attractive. A young woman stated:

"People think that a 'light' pizza with $50 \%$ less fat is healthy. But if you look closely at the ingredients, they add a lot of other stuff. (...) We think 'light' means healthy, but I don't think that's always the case."

\section{Identifiability of low-calorie choices}

Another condition for the effectiveness of interventions that emerged was that they should make low-calorie choices evident to consumers. Participants regularly stressed the importance of clear nutritional information on food packages, especially for those who have insufficient knowledge of nutritional values. On-pack information should be clear enough for all consumers to make an informed decision. As a woman with a higher education said:

"You can't expect consumers to be experts in every area. Even I have problems reading the product information. Packaging should give clear information on nutritional values."

Participants showed a strong preference for the use of food labels that carry the traffic-light system to indicate nutritional values. Such colourful information would make identification of low-calorie choices easier. Some participants believed that the government should force food suppliers to provide food labels with the traffic-light system:

"I really like the use of colours. It's very easy to use. When you see the red colour, you know it's bad for you, and if it's green then it's good (...) This system should be mandatory."

The quote above, given by a mother of two children, reveals that the perceived effectiveness of educational interventions was also associated with the ease-of-use of information. Some participants complained about the complexity of the information on product packages, which impedes the identification of healthy choices. Virtually all who addressed the ease-of-use of information agreed that educational interventions should force information to be simple, comparable, and uniform. 


\section{Perceived effectiveness of combinations of intervention strategies}

In addition to the assessment of separate interventions, participants regularly discussed the effectiveness of combinations of interventions. Most thought that combining educational, marketing, and legal interventions would more effectively stimulate consumers to make low-calorie choices than each intervention separately. Some participants pointed at the success of books on dieting, thereby claiming that educational interventions will be more effective when they are combined with marketing strategies. Furthermore, virtually all thought that tax measures would be more effective if subsidies were provided at the same time:

"It would be good to not only make the bad things more expensive, but also to make the good things cheaper. That way it remains balanced. To me, that seems more effective."

\section{Perceived fairness of intervention strategies for low-calorie food choices}

Figure $2.2 \mathrm{c}$ shows the perceived fairness of the nine archetypical interventions. The following section describes the six aspects that can be discerned with respect to the perceived fairness of interventions.

\section{Fairness of encouragement versus discouragement of choices}

First, the distinction between encouragement and discouragement of choices appeared to be relevant for the perceived fairness of interventions. Participants regularly discussed whether interventions should focus on encouragement of lowcalorie choices or on discouragement of high-calorie choices. Figure $2.2 \mathrm{c}$ indicates that perceptions of fairness not only varied across interventions, but also within interventions. Overall, encouraging strategies were rated slightly more fair than the discouraging counterparts (1st, 3rd, and 5th versus the $2 \mathrm{nd}$, 4th, and 6th). When asked to account for the preference of encouraging strategies, a woman claimed:

"Rewarding healthy actions is fair. Interventions should be structured in a positive manner."

In contrast, proponents of discouraging interventions often pointed at the effectiveness of discouragement to illustrate why they thought these interventions were fair. While arguing that taxes for high-calorie foods are a fair way of stimulating low-calorie choices, a formerly obese man stated: 
"To make people think about and change their food choices, you have to hit them where it hurts to be effective: in their wallet."

In addition, similar to the perception that combinations of interventions increase effectiveness, tax measures for high-calorie foods were also perceived fairer when they were paired with subsidies for low-calorie foods.

\section{Societal fairness of intervention strategies}

Second, to assess the fairness of interventions, participants took potential consequences for society into consideration. The perceived monetary costs and benefits of intervention strategies caused a differentiation in appraisal of fairness. A few participants questioned the fairness of governmental food-education campaigns. They claimed that these campaigns are not effective in combating the obesity problem and are therefore not an efficient use of community resources. A majority of participants, however, contested this view by claiming that a lack of knowledge lies at the heart of the obesity problem. They stressed that extensive food choice education is a fair way of stimulation; some even argued that it is an absolute necessity. Furthermore, those in favour of health campaigns emphasised the lower costs for healthcare when people would more often make low-calorie food choices.

The consequences of legal interventions were also much debated. Participants particularly addressed the effects of taxes and subsidies on society. While virtually all perceived subsidies to be a fair way to stimulate low-calorie food choices because they decrease consumers' expenses, this was different for taxes. Those who thought taxing of high-calorie foods was fair argued that increasing the prices is profitable for the government, while it also stimulates consumers to make low-calorie choices more often. Opponents, in reaction, were suspicious of the use of the tax revenues and pointed at the conflicting roles the government plays with regard to legal interventions:

"The government says you shouldn't smoke, but at the same time they expect to generate large amounts of money by immensely taxing the cigarettes. The taxing of food is exactly the same."

\section{Fairness of effects on consumer groups}

Third, participants associated fairness with the implications that interventions have for specific groups of consumers. Many thought that excessive taxing of high-calorie foods would heavily burden people with a low budget. As a result, these people 
would have fewer resources available for participation in social happenings, sports, and other leisure activities. Participants therefore feared for social exclusion of that group, which they regarded as an unfair consequence of the intervention.

Children were another specific group that was addressed. Participants with children often voiced concerns regarding the aggressive marketing of unhealthy foods towards kids. Some therefore favoured a legal restriction of the promotion of unhealthy foods towards children. In contrast, these participants welcomed marketing and promotion when it concerned healthy foods like fruit and vegetables. As a result, a majority agreed that it would be fair to implement marketing and educational interventions to stimulate children to choose low-calorie foods. Some even stated that both teaching children about the origins of foods and providing information about healthy eating should be implemented in school programs.

\section{Fairness of effects on the food industry}

In addition to the effects on consumer groups, consequences for the food industry were also taken into account. Some participants felt that hindering the food industry too much with interventions would be unfair. They perceived legal interventions such as taxing and restricting advertising to be unfair if they threaten companies' existence. During one interview, an elderly man felt that farmers would be unfairly cornered due to certain interventions:

"I have an agricultural background and I know how hard it is for farmers to make a living. Some legal interventions would make that even harder and that would not be fair."

When talking about the effects of interventions on the food industry, participants also pointed at potential undesired side effects for consumers. Some feared that, as a result of mandatory traffic-light labelling, food manufacturers would manipulate food ingredients. This manipulation could compromise the healthiness of low-calorie products:

"A drawback of that intervention (traffic-light labelling) is that there is a danger of manipulation with ingredients. Food suppliers will do anything to get the red dots off their packages." 


\section{Fairness of disseminated information}

Fifth, participants often discussed the fairness of educational interventions. The accuracy of both presently existing ways of nutritional disclosure and the ones proposed by the interventions was heavily contested. The fairness of such interventions therefore was questioned. A large number of participants felt that food suppliers sometimes make inaccurate health claims with regard to their products. Identical sentiments were expressed with regard to the clarity of product content information. Participants regularly complained about the complexity of information on food packaging. Some displayed frustration towards food manufacturers by pointing at the numerous E-numbers (chemical additives) they put on the ingredient lists. Others participants pointed at unpronounceable names they encounter when reading information on food packages. Therefore all were in favour of more comprehensible information on food packages.

To increase the fairness of educational interventions, the majority of participants agreed that the source of such strategies needs to possess extensive knowledge of nutrition and health claims. In addition, the source of interventions should be autonomous and independent of the food industry. Because the current labels in the Netherlands were introduced by the food industry, many participants disapproved of existing food labelling systems.

\section{Perceived liberty, autonomy, and privacy of food choice}

Last, a large number of participants were afraid that interventions would threaten their liberty, autonomy, and privacy. The paternalistic nature of both taxing highcalorie foods and restricting high-calorie food advertising led to varying opinions regarding fairness. Participants who perceived themselves to be solely responsible for food choice thought that consumers should not be 'told' what is best for them because that would imply that they are incapable of making choices on their own. As one elderly man firmly stated:

"It is starting to look like a dictatorial situation. Discouraging all the unhealthy stuff and fill the streets with healthiness propaganda (...) If we can't freely choose to enjoy unhealthy things, then our society is doomed."

Others who did acknowledge the additional responsibility of other stakeholders viewed governmental participation as an important condition for the legitimacy of interventions. Those participants considered it fair to be patronised to a certain degree as long as they were not forced to make a certain choice: 
"Whether or not you want to make unhealthy choices, it still is your own choice. And if these interventions navigate you to healthier choices without forcing you, then that's good for everyone."

Participants reached consensus on the fact that interventions should not threaten the freedom of choice. All stressed that being able to make the food choices you want without being restricted is a great good in a free society.

Related to the discussion about free choice was the issue of privacy. Participants considered interventions that register personal food choices to be an invasion of privacy. An employer tracking food choices in the worksite cafeteria to give feedback on the amount of calories employees buy was therefore seen as unfair:

"I don't want my employer to know what and how much I eat and drink. That's none of their business."

\section{Re-assessing acceptability}

During the last part of the interviews and focus group discussions participants once more rated their acceptance of the interventions. Looking at differences between the first and second classification of acceptance in Figures 2.2d, it becomes apparent that making high-calorie choices more expensive became less acceptable on second thought. When the moderator asked why that intervention had become less acceptable, a wealthy woman stated:

"First I didn't really have a problem with it. But I realised that to some others it's just not fair to tax foods."

Besides a noticeable change in acceptance of taxing high-calorie foods, participants reported no major shifts when comparing the first and second classifications of acceptability. Neutral ratings, however, had declined for the majority of the interventions during the second classification. Most participants who reported less neutral ratings classification attributed this difference to the exchange of ideas and opinions with others. The sharing of arguments for and against acceptance of interventions helped many to form an opinion. A man indicated:

"There are no big differences. And the things that did change, I did that because some good arguments were brought forward by others in this group." 


\subsection{Discussion}

The current study confirms existing literature concerning the beliefs that influence consumer acceptance of intervention strategies (Eriksson et al., 2008). Furthermore, the underlying aspects related to these beliefs are identified for interventions in the food domain. We show that both general beliefs regarding obesity and interventionspecific beliefs regarding the effectiveness and fairness are associated with consumer acceptance of interventions for low-calorie food choices. The general beliefs regarding obesity concern issues of responsibility for food choice and problem awareness. Intervention-specific beliefs, on the other hand, deal with statements about why interventions are (not) effective and why the interventions are (not) a fair way of stimulating low-calorie choices.

The majority of quotes that were given during the discussions on acceptability consisted of comments on the effectiveness and the fairness of interventions. In addition to this finding, participants showed no major differences between the classifications of acceptability in the beginning and at the end of the discussions. These two findings strengthen the claim that acceptability, perceived effectiveness, and perceived fairness are interrelated beliefs with regard to interventions in the food domain.

Participants were aware that obesity numbers have risen over the past three decades. The majority attributed this phenomenon to a food environment that encourages overeating, much like the objectively observed 'obesogenic environment' in literature (Giskes, Van Lenthe, Avendano-Pabon, \& Brug, 2011). This attribution explains the finding that a majority of participants thought most interventions were acceptable.

In addition, the perceived responsibility for food choice was related to the acceptance of interventions. While all study participants acknowledged a personal responsibility for their food choice, they did not agree on how much others, particularly the government and food suppliers, were also responsible. Those who thought such third parties were also responsible were more likely to show acceptance of interventions. These findings agree with Chambers and Traill (2011) and Barry et al. (2009), who found that the support for obesity prevention policies was greatest when causes for obesity rates were attributed to factors beyond individual control. 
Literature on the effectiveness of interventions suggests that strategies that discourage high-calorie choices are more effective than strategies that encourage low-calorie choices (Epstein et al., 2010; Giesen, Havermans, Nederkoorn, \& Jansen, 2012). Many participants, however, viewed interventions that encourage low-calorie choices to be more effective. To identify a rationale for this discrepancy, the relation between the perceived effectiveness and the actual effectiveness of interventions needs to be studied. For instance, it would be interesting to see what happens when evidence for effectiveness is provided, especially because positive statements regarding effectiveness seem to increase acceptance of interventions (Promberger, Dolan, \& Marteau, 2012).

Legal interventions, and thus governmental involvement in interventions, remained a controversial issue throughout the discussions. This controversy appeared to be twofold. First, fairness classifications of legal interventions differed between participants. Second, the discussions showed a discrepancy between the perceived fairness and the perceived effectiveness of legal interventions. Even though roughly half of the participants perceived legal interventions to be unfair, many did see the government as the most capable source to intervene effectively. Experts believe that legal interventions and governmental involvement are indispensable when it comes to reducing obesity rates (Faulkner et al., 2011). This sentiment has been echoed in many other studies (Gortmaker et al., 2011; Magnusson, 2010; Swinburn et al., 2011). To increase acceptance of governmental policies in the food domain, concerns about governmental involvement therefore need to be addressed.

Child-focused interventions show great promise in reducing childhood overweight and obesity (Doak, Visscher, Renders, \& Seidell, 2006). Participants expressed similar opinions by emphasizing the importance of child-focused interventions. Many thought that educational and marketing interventions both at schools and at home were acceptable and effective strategies to stimulate children to choose low-calorie foods. Participants saw parents as the most appropriate source to implement interventions for their children because they have both the responsibility and the opportunity to stimulate children to choose low-calorie foods. This finding is similar to Mitchell et al. (2013), who stress the importance of interventions aimed at parents to improve children's eating behaviour.

With regard to the generalizability of the findings, two issues are worth mentioning. First, the results merely reflect the viewpoints of a sample of Dutch consumers who varied with regard to age, gender, and income. It is possible that variations in political 
and cultural circumstances lead to different levels of acceptance as well as other mechanisms underlying acceptance. For instance, Mazzocchi et al. (2014) found differing levels of acceptance of obesity prevention policies across five European countries, presumably due to the familiarity with specific policies. However, with respect to the mechanisms underlying acceptance, we think that the concepts that we identified are quite generalizable, particularly because these also underlie acceptance of interventions in non-food domains (Eriksson et al., 2008).

Second, this study limited its scope to acceptance of interventions for consumer choices in terms of caloric value. It would be extremely useful to see whether consumers' perceptions are construed similarly when talking about interventions that target other product characteristics, e.g. increased fruit consumption or decreased salt consumption. Similar to the expected generalizability of the concepts underlying acceptance across cultures, we expect them to be generalizable to acceptance of interventions aimed at other nutritional characteristics as well.

With respect to the methods that were used, again two issues are worth mentioning. First, a potential limitation is that a small number of intervention strategies were used to assess consumer acceptance. However, the interventions were carefully selected on the basis of social marketing literature (Van Trijp et al., 2005) to represent different types of interventions (education, marketing, and law). The use of nine archetypes also facilitated the possibility of exploring participants' acceptance of these generic interventions as well as more detailed versions of these interventions (e.g. specific locations and sources). Furthermore, participants could have experienced confusion when a larger number of interventions would have been used.

Second, one can argue that the order of the different parts of the discussions led to modifications in participants' ratings of acceptance. Because this sequence was not varied, potential biases, particularly order effects (Bowling, 2005) and belief overkill (J. Baron, 2009) cannot be ruled out. Looking at participants' argumentations for their ratings, we have little reason to believe that these side effects indeed surfaced and compromised the legitimacy of participants' responses. Furthermore, the specific order was employed to prevent that discussions on effectiveness and fairness of interventions would influence the initial rating and discussion on acceptance. This enabled us to see whether statements about effectiveness and fairness would surface spontaneously, like they did. 
An important last note is that our research emphasises interventions that focus on calorie intake rather than calorie expenditure. We chose this emphasis because literature stresses that curtailment of calorie intake is of greater importance to reduce obesity rates (Cohen et al., 2010). That does not mean, however, that interventions that target calorie expenditure should receive less attention, particularly because those kinds of interventions also show great promise in reducing obesity rates (O'Hara et al., 2013). Future research concerning consumer acceptance of interventions for obesity prevention should therefore also include interventions that emphasise calorie expenditure.

\subsection{Conclusions}

Policymakers in the food domain need to be able to anticipate consumer acceptance of intervention strategies. Knowledge of factors that influence consumer acceptance therefore is crucial. The present study identifies the beliefs and underlying aspects that influence acceptance of interventions from a consumer perspective. The findings can be used by policymakers to anticipate consumer reactance towards interventions and to negotiate the development and communication of new strategies.

The present article enriches existing literature on food choice interventions in two ways. First, it confirms research on the beliefs that influence acceptance of interventions from other domains. Second, and most important, it identifies the underlying aspects of these beliefs specifically for food choice interventions. Besides consensus on a few issues, the outcomes show that consumers' classifications of acceptance, perceived effectiveness, and perceived fairness of interventions differ between and within individuals. Logical next steps would be to segment people based on how they perceive specific interventions and to explore how these segments should be approached to increase acceptance. This will enable us, for instance, to see whether providing evidence for actual effectiveness of interventions will increase the perceived effectiveness and subsequently the acceptance of interventions. 


\subsection{Acknowledgements}

We would like to thank Aleksandra Berezowska and Larissa Shnayder for their contributions to the study. 



\section{HETEROGENEITY IN BARRIERS} REGARDING THE MOTIVATION, THE OPPORTUNITY AND THE ABILITY TO CHOOSE LOW-CALORIE SNACK FOODS AND BEVERAGES Associations with real-life choices

This chapter is published as:

Bos, C., Van der Lans, I., Van Rijnsoever, F., Van Trijp, H.C.M. (2015) Heterogeneity in barriers regarding the motivation, the opportunity and the ability to choose low-calorie snack foods and beverages: associations with real-life choices Public Health Nutrition, 19(9), 1584-1597 doi:10.1017/S1368980015002517 


\section{Abstract}

\section{Background}

Employing Rothschild's Motivation-Opportunity-Ability (MOA) framework, the present study examines the extent to which heterogeneity in barriers regarding the motivation, the perceived opportunity, and the perceived ability to choose lowcalorie over high-calorie snacks is associated with the proportion of low-calorie snack choices in real-life. Furthermore, the study investigates which dominant barrier profiles can be discerned.

\section{Methods}

Data were obtained from a survey about participants' motivation, opportunity, and ability to choose low-calorie over high-calorie snacks and a Food Frequency Questionnaire (FFQ) that measured habitual consumption of snack foods and beverages. Data were analysed using $\mathrm{R}$ packages lavaan and NbClust, and SPSS. Participants reflected a representative sample $(n=1318)$ of the Dutch population based on gender (686 women), age, and education level.

\section{Results}

For both snack foods and beverages, motivation to choose low-calorie over highcalorie snacks was associated strongest with proportions of low-calorie choices. The perceived ability and perceived opportunity were also associated with proportions of low-calorie choices, albeit to a lesser extent. Furthermore, three dominant profiles of barriers were identified: the no-barrier profile, the lack-of-opportunity profile, and the lack-of-motivation profile. These profiles differed significantly on proportions of low-calorie snack choices, daily meal consumption, and sociodemographics.

\section{Conclusions}

Heterogeneity in barriers regarding the motivation, the perceived opportunity, and the perceived ability to choose low-calorie over high-calorie snacks is associated with the proportion of low-calorie snack choices in real-life. By identifying and appreciating heterogeneity in barriers, the present study provides further incentives for the tailoring of intervention strategies. 


\subsection{Introduction}

A worrying rise of overweight and obesity rates, accompanied by related chronic and non-communicable diseases, continues to threaten public health around the world (Swinburn et al., 2011). The origin of overweight and obesity lies primarily in an imbalance between the intake and the expenditure of energy (Bleich et al., 2008). During the last decades there has been an increase in daily eating occasions (Popkin \& Duffey, 2010) and therewith daily energy intake has also increased.

The excess energy intake is primarily a consequence of a change in snack consumption. In the USA, energy intake through regular meals increased slightly from 1977 to 2006 (8\%), while the energy intake through snacks more than doubled (130\%) (Popkin \& Duffey, 2010). Similar trends have been detected in Europe (Gracia \& Albisu, 2001; Ovaskainen et al., 2005). Since energy expenditure decreased only slightly over the same period (Bleich et al., 2008), the obesity problem is best described as an issue of systematic caloric overconsumption (Shelley, 2012), with snacking as an increasingly important contributor.

Literature on the relationship between weight status and snacking behaviour is inconclusive (Johnson \& Anderson, 2010). This can partly be explained by the fact that weight status is not only influenced by snacking but also by factors such as meal consumption and physical activity. Furthermore, these factors are interrelated, as sometimes people eat a snack as meal replacement, or because of some heavy physical activity. At a methodological level, an additional reason might be the lack of consensus on the definition of snacking. This makes it difficult to establish a relation, since conclusions about the relation between obesity and snacking are heavily dependent on how snacks and snacking behaviour are defined (Gregori, Foltran, Ghidina, \& Berchialla, 2011; Johnson \& Anderson, 2010). The present study therefore adapts the proposed universal definition of snacks by Johnson et al. (2010): everything that is consumed outside the regular meals (breakfast, lunch, and dinner), and is not a substitute for a meal.

Whether snacking is detrimental to one's weight status depends on the caloric values of frequently consumed snacks. In itself snacking does not necessarily promote weight gain, because low-calorie snacks that supplement a balanced and healthy diet are available (Hartmann, Siegrist, \& van der Horst, 2013). However, most foods and drinks that are consumed outside the regular meals are high in calories (Zizza, SiegaRiz, \& Popkin, 2001). To effectively stimulate low-calorie snacking, insight in the 
determinants of choosing snacks that are low in calories over snacks that are high in calories is crucial.

The present study employs Rothschild's Motivation-Opportunity-Ability (MOA) framework (1999) and examines the extent to which heterogeneity in barriers regarding the motivation, the perceived opportunity, and the perceived ability to choose low-calorie over high-calorie snacks is associated with the proportion of lowcalorie snack choices in real-life. We expect that on a theoretical level the association between barriers and real-life choices is generalizable across snack categories, even though some research suggests that differences in barriers for low-calorie choices within these snack categories might exist (Elbel, 2011). To test generalizability across snack categories, barriers and real-life choices are assessed separately for snack foods and beverages. Furthermore, the study investigates whether subgroups of people with similar barrier profiles can be found. Identification of dominant barrier profiles enables tailoring of intervention strategies, which has shown to be beneficial for both the acceptance and effectiveness of interventions (Bos, Van der Lans, Van Rijnsoever, \& Van Trijp, 2013; Gordon et al., 2006).

\subsection{Theoretical background}

Snack choices are an element of the total spectrum of food choices. Hence, to gain insight in the determinants of choosing low-calorie over high-calorie snacks, we build from the literature on food choices in general. Food choices are frequent, multifaceted, situational, dynamic, and complex (Sobal \& Bisogni, 2009) and therefore reasons for choosing certain foods vary between people. We will provide a short overview of two categories of behavioural determinants that are important for food choices: individual-level and environmental determinants.

Several behavioural theories that have been applied to dietary behaviours, such as the Theory of Planned Behaviour (TPB) (Ajzen \& Timko, 1986), the Health Belief Model (HBM) (Rosenstock et al., 1988), and the Trans-Theoretical Model (TTM) (Greene et al., 1999), recognize evaluations of expected outcomes of behaviour as key determinants for food-choices. These evaluations, referred to as attitudes in the TPB, can be divided into cognitive and affective attitudes (Crites, Fabrigar, \& Petty, 1994). Cognitive attitudes are evaluations of expected utilitarian outcomes of dietary behaviours (e.g. "I think eating low-calorie snacks is healthy"), whereas affective attitudes reflect evaluations of expected hedonic outcomes of choosing certain 
foods (e.g. "I think eating low-calorie snacks is boring"). When people's cognitive and affective attitudes towards choosing certain foods are negative, they will be less likely to choose these foods (Conner et al., 2002; Geeroms, Verbeke, \& Van Kenhove, 2008a; Satia, Kristal, Curry, \& Trudeau, 2001). In addition, the TPB claims that normative beliefs regarding dietary behaviour are also important.

Most behavioural theories furthermore acknowledge that perceptions about having sufficient skills and knowledge to perform a behaviour (HBM and TTM: self-efficacy; TPB: perceived behavioural control) influences the likelihood of execution of that behaviour (e.g. "I am confident that I can differentiate between low-calorie and highcalorie snacks"). When people feel that they lack these abilities, and thus have a low self-efficacy, they are less likely to engage in the behaviour (Hollywood et al., 2013; Mai \& Hoffmann, 2012).

In contrast to behavioural theories that highlight individual-level influences, Social Ecological Models (SEM) (Catlin, Simoes, \& Brownson, 2003) emphasize environmental factors that drive food choices. The ANGELO framework (Swinburn, Egger, \& Raza, 1999), which has been specifically designed to conceptualize food choice environments, distinguishes between physical-, economic-, and media environments. For the physical environment, research has shown that changes in the physical accessibility and availability of foods have a great impact on food choice behaviour (Dayan \& Bar-Hillel, 2011; Rozin et al., 2011). And although causality is unclear, studies that focused on people's perceptions of food choice environments found that a higher perceived physical availability of energy-dense foods is associated with a higher intake of these foods (Boehmer, Hoehner, Deshpande, Ramirez, \& Brownson, 2007; Giskes, Van Lenthe, Brug, Mackenbach, \& Turrell, 2007; Gustafson et al., 2011; Lucan \& Mitra, 2012). Additionally, food choice experiments have shown that the economic environment heavily influences food choices (Epstein et al., 2010; Giesen et al., 2012). This is in line with research based on interviews and focus groups, where costs of foods and beverages are often mentioned as barriers and triggers for consumption (Dressler \& Smith, 2013; Hartman, Wadsworth, Penny, Van Assema, \& Page, 2013). Lastly, the association between the media environment and food choices has often been suggested in scientific literature, mostly by linking advertising for unhealthy foods to obesity (Lobstein \& Dibb, 2005; Neumark-Sztainer et al., 1999). Evidence for the influence of the physical, economic, and media environment on food choices has accumulated over the past years, thereby implying that increasing obesity rates are primarily a result of an 'obesogenic environment' that promotes overeating (Giskes et al., 2011; Kirk, Penney, \& McHugh, 2010). 
Summarizing, behavioural theories that have been applied to dietary behaviours show that both individual-level and environmental determinants drive food-choice behaviour. Rothschild's Motivation-Opportunity-Ability (MOA) framework (1999) encompasses the heterogeneity in determinants by stressing the significance of both individual-level and environmental influences on behaviour. The MOA framework integrates the core concepts of the TPB and SEM, which in combination have been identified as most promising models for obesity prevention (Baranowski et al., 2003).

Applied to the food choice context, the framework theorizes that when motivation, opportunity, and ability for a certain dietary behaviour are sufficiently present, people will likely execute the behaviour. A lack of motivation, opportunity, and/or ability, however, functions as a barrier for behaviour. More specifically, a lack of motivation results in a resistance to behave, whereas the lack of opportunity and ability result in an inability to behave.

Rothschild's MOA framework poses that there is heterogeneity in barriers towards desirable behaviour, i.e. people differ regarding the extent to which they experience the various barriers. It structures this heterogeneity by distinguishing between a total of eight different profile types based on the presence or absence of barriers within MOA (Motivation: barrier/no barrier $x$ Opportunity: barrier/no barrier $\mathrm{x}$ Ability: barrier/no barrier). The framework then argues that, depending on the dominant barrier profiles, intervention strategies based on education (for Abilityrelated barriers), marketing (for Opportunity-related barriers), and/or law (for Motivation-related barriers) should be used to diminish barriers for behaviour.

The present study consists of two parts. The first part relates heterogeneity in barriers in motivation, perceived opportunity, and perceived ability to the proportion of low-calorie snack choices in real-life. The second part examines which and how many of the eight MOA profile types, as suggested by Rothschild, are most prevalent. Public health policies greatly benefit from identification of actionable subgroups with similar barrier characteristics, as providing tailored interventions to each individual is infeasible. By identifying these dominant MOA profiles, recommendations regarding intervention strategies can be made. 


\subsection{Methods}

\section{Procedure and sample}

An online survey, comprising of two waves, was conducted. Wave 1 was divided into two parts (presented in a random order): one part focused on individuals' motivation, opportunity and ability to choose low-calorie over high-calorie snack foods, while the other part focused on beverages. During the instructions of Wave 1 , respondents were given the following information: 'Low-calorie snacks contain relatively few calories. For instance fruits and rice waffles/water and light soft drinks. High-calorie snacks contain relatively many calories. For instance crisps and pie/chocolate milk and regular soft drinks'. Four weeks later the second wave of data was collected, which consisted of an FFQ specifically aimed at snack foods and beverages. Both in the first and second wave respondents were instructed to think of snacks as being 'all foods and beverages that are consumed outside breakfast, lunch and dinner'. Splitting up the data collection into two waves was done to prevent respondent burden and the four-week intermission was employed to minimize consistency bias.

Data were collected in the Netherlands by the commercial marketing research agency GfK. GfK provided a representative sample of the Dutch population based on gender, age and education level, which was recruited from the GfK Online Panel. The incentive to participate was a number of 'GfK points' for which coupons can be ordered (equivalent to about $5 €$ ). Of the 1573 respondents who completed Wave 1 , a total of 1318 completed Wave 2 . Response rates were $70 \%$ and $84 \%$, respectively. Characteristics of these 1318 respondents are summarized in Table 3.1. 
Table 3.1: Characteristics of respondents

\begin{tabular}{lrr}
\hline & $\begin{array}{c}\text { Males } \\
(\mathbf{n}=632)\end{array}$ & $\begin{array}{r}\text { Females } \\
(\mathbf{n}=686)\end{array}$ \\
\hline Age (\%) & & \\
18-34 years & $26.4 \%$ & $27.0 \%$ \\
35-49 years & $33.7 \%$ & $31.3 \%$ \\
50-65 years & $39.9 \%$ & $41.7 \%$ \\
Education $(\%)^{1}$ & & \\
Low & $26.3 \%$ & $29.9 \%$ \\
Middle & $42.2 \%$ & $43.3 \%$ \\
High & $31.3 \%$ & $26.8 \%$ \\
BMI $(\%)$ & & \\
Underweight $\left(<20 \mathrm{~kg} / \mathrm{m}^{2}\right)$ & $2.2 \%$ & $2.8 \%$ \\
$\quad$ Normal weight $\left(20-25 \mathrm{~kg} / \mathrm{m}^{2}\right)$ & $41.0 \%$ & $46.6 \%$ \\
Overweight $\left(25-30 \mathrm{~kg} / \mathrm{m}^{2}\right)$ & $43.5 \%$ & $30.0 \%$ \\
Obese $\left(>30 \mathrm{~kg} / \mathrm{m}^{2}\right)$ & $15.5 \%$ & $20.6 \%$ \\
\hline 1Based on the official Dutch education classification ${ }^{1}$
\end{tabular}

\section{Measures}

Motivation, opportunity and ability to choose low-calorie over high-calorie snacks Because the MOA framework itself does not dictate how the MOA constructs should be measured, we used and adapted scales from existing scales from the behavioural theories (Brug, 2008; Van Trijp et al., 2005) as much as possible. When appropriate scales were lacking, we constructed them on the basis of previous qualitative research (Bos et al., 2013; Krukowski, McSweeney, Sparks, \& West, 2012). A complete list of items is provided in the online supplementary material.

For measuring Motivation, participants' attitudes, personal norms and social norms towards choosing low-calorie over high-calorie snacks were assessed. Cognitive and affective attitudes towards choosing low-calorie over high-calorie snacks were measured by means of eight 7-point semantic differential scale items, which were adapted from Conner et al. (2002) and Crites et al. (1994). Personal norms regarding choosing low-calorie over high-calorie snacks were assessed by three items, adapted from Parker et al. (1995). Social norms were measured with one item that was adapted from Conner et al. (2002).

\footnotetext{
${ }^{1}$ http://www.cbs.nl/NR/rdonlyres/7C94DE33-621C-4355-928A-

8B90F9F5D777/0/2006soiniveauindeling201213.pdf
} 
For measuring Opportunity to choose low-calorie over high-calorie snacks ten items were constructed. The items were based on previous qualitative research (Bos et al., 2013; Krukowski et al., 2012) and were structured according to the ANGELO framework (Swinburn et al., 1999), which differentiates between the physical, economic and media environment in which food choices are made. Using this subdivision of environments, participants' perceptions of the physical and economic environments were assessed with four items each. Additionally, two items represented perceptions of the media environment.

For measuring Ability, participants' self-efficacy with regard to having the basic skills and knowledge to choose low-calorie over high-calorie snacks was assessed. These basic skills and knowledge were identified in a previous qualitative study (Bos et al., 2013). For knowledge, we constructed four items that assessed the perceived ability to distinguish between low-calorie and high-calorie products. Skills to understand calorie information on packages were assessed with one item. The formulation of the self-efficacy items was adapted from Sheeshka et al. (1993).

The surveys were pre-tested during cognitive walkthrough interviews $(n=5)$. Based on these pre-tests, improvements in the formulation of items were made. The revised surveys were then piloted online $(n=50)$. Based on similar feedback from four respondents, two minor changes were made regarding the qualification of behavioural ability to assess calorie content from 'well' to 'accurately'. Because this was the only feedback on item formulation from the pilot study, we judged the final questionnaire items to be adequately formulated.

\section{FFQ and main meal consumption}

To assess real-life snack choices, an FFQ was administered. The questionnaire was carefully developed with the considerations regarding the construction of FFQ's in mind (Cade, Thompson, Burley, \& Warm, 2002). Participants indicated how often they normally choose specific types of snacks: less than once per month/never (coded as 0 times/week), 1-3 times per month (coded as 0.5 times/week), 1-3 times per week (coded as 2 times/week), 4-6 times per week (coded as 5 times/week), daily (coded as 7 times/week) and several times per day (coded as 14 times/week). The snack foods and beverages that were included were identified as frequently consumed snacks in previous research (Bos et al., 2013). For low-calorie snack foods these were (fruit) biscuits, small candy bars, gingerbread, water ice, soup, crackers and fruit; for high-calorie snack foods these were cake, cookies, regular candy bars, chocolate, pie, ice cream, warm snacks, nuts, crisps and sandwiches. Low-calorie 
beverages consisted of coffee, tea, (flavoured) water, fruit juice light, soft drink light and malt/light beer; high-calorie beverages consisted of cappuccino, chocolate milk, fruit juice regular, soft drink regular, milk, yoghurt drink, regular beer, wine and liquor. Items were classified as low-calorie when a portion was $<418.4 \mathrm{~kJ}$ ( $<100 \mathrm{kcal})$ and high-calorie when a portion was $>418.4 \mathrm{~kJ}$ (>100kcal), which is similar to the classification by Kocken et al. (2015). This classification of items was not made explicit to participants. We obtained portion sizes and caloric values from the website of the Dutch Nutrition Center ${ }^{2}$. The proportion of low-calorie choices was calculated by dividing the total low-calorie choices by the total low- and high-calorie choices combined. This was also done for individual products with both low-calorie and high-calorie versions (e.g. soft drinks). An overview of FFQ items can be found in Tables 3.2 and 3.3.

Main meal consumption patterns were identified by asking participants how often they normally consume breakfast, lunch and dinner: less than once per month/ never, 1-3 times per month, 1-3 times per week, 4-6 times per week, daily and several times per day. Main meal consumption patterns were included because they are known to be associated with snack consumption (Hartmann et al., 2013). Because the majority of participants did consume main meals daily (ranging from 78.1\% for breakfast to $92.4 \%$ for dinner), the items were dichotomized (e.g. daily breakfast: yes/no).

\footnotetext{
${ }^{2}$ (http://www.voedingscentrum.nl/nl/schijf-van-vijf/eet-gevarieerd/hoeveelcalorieen-zitten-erin.aspx)
} 
Table 3.2: Mean weekly consumption of snack food FFQ items

\begin{tabular}{llrrr}
\hline Category & Snack food items FFQ & $\%^{1}$ & Mean & SD \\
\hline Sweet snacks & Low-calorie & & & \\
& (Fruit)biscuit & 24.0 & 0.97 & 1.84 \\
& Small candy bar/chocolate & 25.9 & 1.01 & 1.73 \\
& Gingerbread & 29.6 & 1.25 & 2.07 \\
& Water ice & 8.8 & 0.42 & 1.21 \\
& High-calorie & & & \\
& Cake and cookies & 39.9 & 1.69 & 2.51 \\
& Regular candy bar/chocolate & 15.9 & 0.63 & 1.27 \\
& Pie & 7.6 & 0.43 & 0.73 \\
& Ice cream & 9.3 & 0.46 & 1.14 \\
Savoury snacks & Low-calorie & & & \\
& Soup & 23.1 & 0.90 & 1.61 \\
& Crackers & & \\
& High-calorie & 26.9 & 1.17 & 2.20 \\
& Warm snacks & & & \\
& Nuts & 12.7 & 0.50 & 0.87 \\
& Crisps & 29.1 & 1.14 & 1.88 \\
Fruits and vegetables & 35.3 & 1.14 & 1.49 \\
& Sandwich & 40.6 & 2.86 & 4.33 \\
& Fruits & 82.2 & 5.73 & 4.58 \\
& Vegetables & 58.9 & 3.62 & 3.93 \\
\hline
\end{tabular}

1 Percentage of participants who consumed the snack food $1 \geq$ per week

${ }^{2}$ These included regular crackers, rice crackers, and rusk

'These included typical Dutch snacks ('kroket' and 'frikadel'), and pizza slices 
Table 3.3: Mean weekly consumption of beverage FFQ items

\begin{tabular}{lllll}
\hline Category & Beverage items FFQ & $\%^{1}$ & Mean & SD \\
\hline Hot beverages & Low-calorie & & & \\
& Coffee & 76.1 & 8.62 & 6.01 \\
& Tea & 71.9 & 6.79 & 5.81 \\
& High-calorie & & & \\
& Cappuccino & 28.3 & 1.92 & 3.84 \\
& Chocolate milk & 10.2 & 0.56 & 1.65 \\
Cold beverages & Low-calorie & & & \\
& (Flavoured) water & 68.4 & 6.82 & 6.00 \\
& Fruit juice light & 19.8 & 0.95 & 2.28 \\
& Soft drink light & 32.1 & 2.20 & 4.03 \\
& High-calorie & & & \\
& Fruit juice regular & 33.6 & 1.65 & 2.84 \\
& Soft drink regular & 28.5 & 1.59 & 3.22 \\
& Milk & 46.7 & 3.05 & 4.04 \\
& Yoghurt drink & 21.3 & 1.08 & 2.38 \\
Alcoholic beverages & Low-calorie & & & \\
& Malt/light beer & 4.3 & 0.21 & 1.07 \\
& High-calorie & & & \\
& Regular beer & 22.3 & 0.92 & 2.10 \\
& Wine & 25.3 & 1.11 & 2.29 \\
& Liquor & 8.1 & 0.39 & 1.42 \\
\hline
\end{tabular}

${ }^{1}$ Percentage of participants who consumed the beverage $1 \geq$ per week

\section{Data analysis}

Data were analysed in three subsequent steps. First, because both existing measures and self-constructed measures were used, we performed confirmatory factor analysis and assessed internal consistency for the measures for the motivation, the opportunity and the ability to choose low-calorie over high-calorie snacks using the R package lavaan (version 0.5-17) (Rosseel, 2012). The confirmatory factor analysis assessed the goodness-of-fit on four indices (cut-off values were adapted from Hair et al. (2010)): (i) the comparative fit index (CFI; good if >0.95); (ii) the Tucker-Lewis index (TLI; good if >0.95); (iii) the root mean-square error of approximation (RMSEA; good if <0.07); and (iv) the standardized root-mean-square residual (SRMR; good if $<0.08$ ). First, measurement fit was investigated for the one-factor models. Then the overall multi-factor measurement model was evaluated, to ensure that the items loaded on their corresponding factor and not on one of the other factors. Internal consistency was evaluated based on $\omega^{2}$ and average variance extracted (AVE). Internal consistency was regarded as satisfactory when $\omega^{2}>0.7$ (Nunnally, 1978) and AVE $>0.5$ (Fornell \& Larcker, 1981). Discriminant validity was regarded satisfactory when the AVE exceeded shared variance with other factors (Fornell \& Larcker, 1981). 
Second, for snack foods and beverages separately we estimated the relationship between the MOA factors and demographic variables, on the one hand, and the proportion of low-calorie choices, on the other, by conducting a linear regression analysis. The relationship was estimated for both the aggregated proportions of lowcalorie choices and proportions of low-calorie choices for individual products with a low-calorie and high-calorie version (e.g. soft drinks). To be able to perform linear regression analysis on the proportions of low-calorie choices, a function of proportions was used: the log odds (Bland \& Altman, 2000). Log odds scores were obtained by taking the logit of the probability of choosing a low-calorie snack (the number of low-calorie snack choices divided by the number of high-calorie snack choices). Because proportions of 0 and 1 have no log odds, these scores were imputed (Tukey, 1962), meaning that proportions of 0 and 1 received the log odds of the lowest and highest proportions (which were $>0$ and $<1$ ) in the data set, respectively.

Third, for snack foods and beverages separately we examined if and how many dominant profiles could be identified based on the motivation, the opportunity and the ability to choose low-calorie over high-calorie snacks. Initially, hierarchical cluster solutions of two to ten profiles were generated using the statistical software package IBM SPSS Statistics 19.0. The hierarchical centroids that were obtained were then used as starting points for k-means clustering. Because the agglomeration schedule was inconclusive in assessing the best cluster solution, the final profile number was determined by using R package NbClust (version 2.0) (Charrad, 2014), which provides several indices for identifying the relevant number of clusters. We subsequently performed ANOVA to identify differences in daily meal consumption and snacking behaviour between the dominant profiles. Within each ANOVA, we ran post hoc comparisons using Tukey's honest significant difference procedure to control the family-wise error rate. 


\subsection{Results}

Psychometric properties of motivation, opportunity and ability measures (Step 1)

Tables 3.4 and 3.5 show that the psychometric properties for the MOA measures were close to identical for snack foods and beverages. Internal consistency was satisfactory both for the snack food and beverage Motivation measures, while discriminant validity was adequate. Within the three Opportunity measures, the AVE and thus the discriminant validity suffered from three oppositely phrased items (one in each of three measures). Removal of two oppositely phrased items for physical Opportunity ('I think that low-calorie snacks, compared to high-calorie snacks, are easy enough to find') and financial Opportunity ('I think that low-calorie snacks, compared to high-calorie snacks, are on sale often enough') resulted in an AVE $>0.5$, thereby reaching satisfactory internal consistency and adequate discriminant validity. Because media Opportunity consisted of only two items, both were kept, even though internal consistency and discriminant validity were not completely achieved. For Ability (knowledge) internal consistency was borderline satisfactory (AVE values of 0.487 and 0.456 ), while discriminant validity was adequate. Because removal of the item with the lowest $R^{2}$ did not result in a better overall $\omega^{2}$ value, we proceeded with all four knowledge items.

Table 3.4 also shows the fit measures for the one-factor models and overall measurement models of the MOA measures for snack foods, with and without the removal of items. The CFI, TLI, RMSEA and SRMR all indicated a good fit for the onefactor models. For the overall measurement model the CFI and TLI were low (0.935 and 0.922), and therefore we tested the measurement model without the two oppositely phrased physical and financial Opportunity items. With the omission of these items, the CFI and TLI changed to acceptable values of 0.964 and 0.956, respectively.

For the MOA measures for beverages (Table 3.5), CFI and SRMR indicated good fit for the one-factor models. The TLI for Ability (knowledge) was slightly lower than the cut-off value (0.947). Similarly, the RMSEA for Opportunity (financial) and Ability (knowledge) were slightly higher than the cut-off (0.074 and 0.077 , respectively). Because these measures were only slightly lower than the cut-off and scored a good fit on the other indices, we evaluated the fit of the one-factor models as acceptable. For the overall measurement model, the TLI was too low (0.941). 
Therefore we again tested the measurement model without the two oppositely phrased physical and financial Opportunity items and found that TLI changed to an acceptable value of 0.967 . Because of the considerable improvements in both internal consistency and overall measurement model fit, we decided to conduct further analysis without the two oppositely phrased physical and financial Opportunity items. 


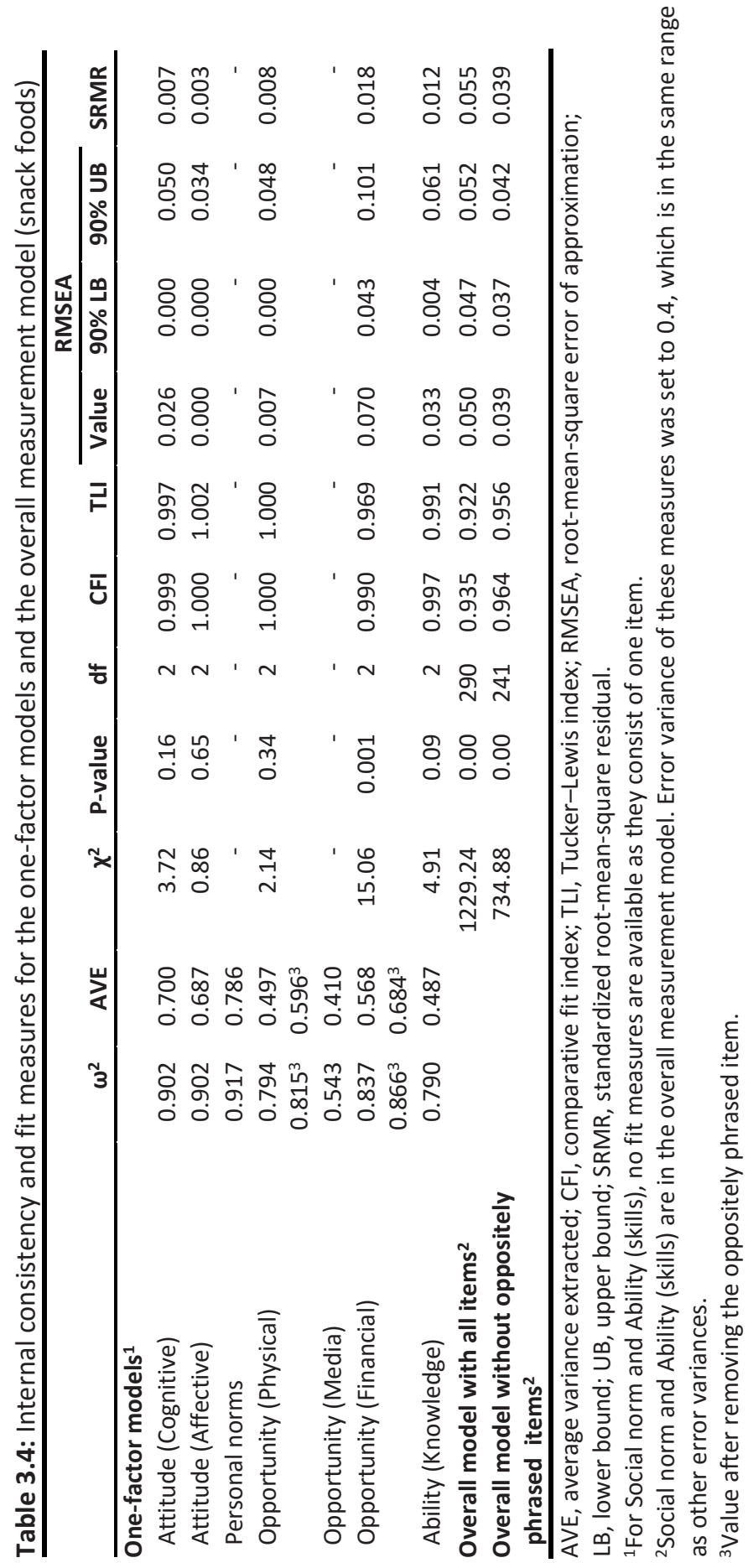




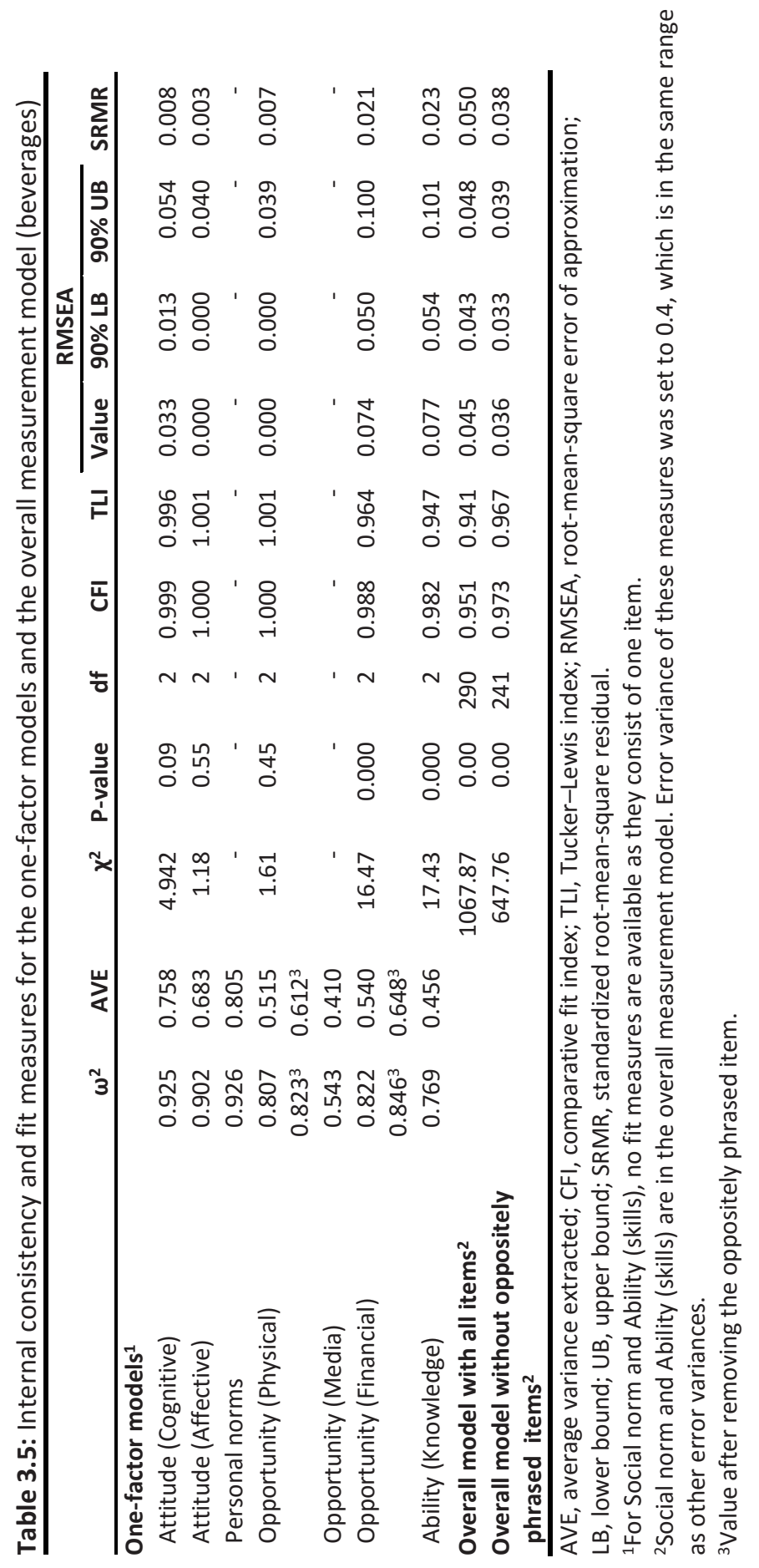


Relationship between motivation, opportunity and ability factors and the log odds of choosing low-calorie snack foods (Step 2)

Table 3.6 shows the outcomes of the linear regression analysis on the log odds of choosing low-calorie snack foods. The model for snack foods, in which we consecutively included the MOA factors for snack foods $\left(R^{2}=0.132\right.$, adjusted $\left.R^{2}=0.126\right)$ and demographic variables $\left(\Delta R^{2}=0.044\right.$, adjusted $\left.\Delta R^{2}=0.042\right)$, accounted for $17.6 \%$ (adjusted $16.8 \%$ ) of the variance in the log odds of choosing low-calorie snack foods.

Table 3.6: Linear regression analysis on the log odds of choosing low-calorie snacks

\begin{tabular}{|c|c|c|c|c|}
\hline & \multicolumn{2}{|c|}{ Snack foods ${ }^{1}$} & \multicolumn{2}{|c|}{ Beverages $^{2}$} \\
\hline & $\beta$ & $\mathbf{P}$ & $\beta$ & $\mathbf{P}$ \\
\hline \multicolumn{5}{|l|}{ MOA Factors } \\
\hline \multicolumn{5}{|l|}{ Motivation } \\
\hline \multicolumn{5}{|l|}{$\overline{\text { Attitude }}$} \\
\hline Cognitive & 0.009 & 0.796 & 0.056 & 0.196 \\
\hline Affective & $0.147 * * *$ & 0.000 & $0.086 *$ & 0.034 \\
\hline Personal norm & $0.162 * * *$ & 0.000 & $0.164 * * *$ & 0.000 \\
\hline Social norm & -0.029 & 0.326 & $-0.073^{*}$ & 0.017 \\
\hline \multicolumn{5}{|l|}{ Opportunity } \\
\hline Physical & -0.054 & 0.138 & 0.046 & 0.221 \\
\hline Media & -0.036 & 0.223 & -0.021 & 0.501 \\
\hline Financial & $0.078 *$ & 0.020 & 0.021 & 0.550 \\
\hline \multicolumn{5}{|l|}{ Ability } \\
\hline Knowledge & -0.005 & 0.836 & -0.026 & 0.330 \\
\hline Skills & $0.102 * * *$ & 0.000 & 0.001 & 0.964 \\
\hline \multicolumn{5}{|l|}{ Demographics } \\
\hline Gender (male) & $-0.115 * * *$ & 0.000 & $-0.106 * * *$ & 0.000 \\
\hline BMI & 0.004 & 0.885 & 0.012 & 0.669 \\
\hline \multicolumn{5}{|l|}{ Age } \\
\hline $18-34$ v. $50-65$ & $-0.106 * * *$ & 0.000 & -0.044 & 0.141 \\
\hline $35-49$ v. $50-65$ & $-0.130 * * *$ & 0.000 & -0.042 & 0.151 \\
\hline \multicolumn{5}{|l|}{ Education } \\
\hline Low v. High & $-0.120 * * *$ & 0.000 & -0.046 & 0.155 \\
\hline Middle v. High & $-0.098 * *$ & 0.001 & -0.026 & 0.409 \\
\hline Daily Breakfast (yes) & $0.058 *$ & 0.050 & 0.049 & 0.103 \\
\hline Daily Lunch (yes) & 0.013 & 0.694 & 0.049 & 0.132 \\
\hline Daily Diner (yes) & 0.026 & 0.379 & 0.027 & 0.379 \\
\hline
\end{tabular}

*P<0.05, ${ }^{* * P}<0.01, * * * \mathrm{P}<0.001$

${ }^{1} R^{2}=0.176$ (adjusted $R^{2}=0.164$ )

${ }^{2} R^{2}=0.126$ (adjusted $R^{2}=0.114$ ) 
Within MOA, significant positive effects on the log odds of choosing low-calorie snack foods were identified for affective attitude, personal norm, financial opportunity and skills in understanding calorie information on packages. Eating breakfast daily also had a positive effect. Women were more likely to choose lowcalorie snack foods, while younger people and people with a lower education were less likely to choose low-calorie snack foods. The $\beta$ values in Table 3.6 indicate the change in log odds. These changes can be translated back into odds ratios (proportion low-calorie divided by proportion high-calorie). The personal norm coefficient of 0.162 , for instance, corresponds to an $18 \%$ increase of the odds of choosing low-calorie snack foods.

Relationship between motivation, opportunity and ability factors and the log odds of choosing low-calorie beverages (Step 2)

Table 3.6 furthermore shows the outcomes of the linear regression analysis on the log odds of choosing low-calorie beverages. The total model for beverages, in which we consecutively included the MOA factors for beverages $\left(R^{2}=0.101\right.$, adjusted $\left.R^{2}=0.095\right)$ and demographic variables $\left(\Delta R^{2}=0.025\right.$, adjusted $\left.\Delta R^{2}=0.019\right)$, accounted for $12.6 \%$ (adjusted $11.4 \%$ ) of the variance in the log odds of choosing low-calorie beverages.

With regard to the MOA factors, positive effects on the log odds of choosing lowcalorie beverages were identified for affective attitude and personal norm, while social norm had a negative effect. Again, women were more likely to choose lowcalorie beverages.

Linear regression analysis was also conducted on the log odds of choosing the lowcalorie version of beverages that have both a high- and a low-calorie version. This included light fruit juice v. fruit juice regular and light soft drink v. regular soft drink. To ensure that only respondents who choose these beverages regularly were included in the regression, a criterion of $>3$ times per week was used. For the log odds of choosing light fruit juice, $R^{2}$ values for $M O A\left(R^{2}=0.104\right)$ and demographics $\left(\Delta R^{2}=0.028\right)$ were similar to the aggregated low-calorie beverage log odds. For soft drinks, the total model accounted for $27.9 \%$ of the variance in the log odds of choosing light soft drinks ( $M O A R^{2}=0.240$, demographics $\Delta R^{2}=0.039$ ). Due to a relatively small number of respondents who drink soda regularly, the adjusted $R^{2}$ amounted to 0.217 and the adjusted $\Delta R^{2}$ to 0.021 . 


\section{Profiles based on motivation, opportunity and ability factors (Step 3)}

Rothschild's MOA framework theoretically differentiates between eight possible profile types based on whether barriers within the motivation, the opportunity and the ability to choose low-calorie over high-calorie snacks were present. Table 3.7 gives the sizes of these eight MOA profile types, which were identified by means of a split on the midpoint of the scales for the averaged motivation, opportunity and ability scores ( $\leq 4=$ no, $>4=y e s$ ), for both snack foods and beverages.

Table 3.7: Percentage of participants with or without barriers regarding motivation, opportunity, and ability to choose low-calorie snacks, based on a score split $(n=1318)$

\begin{tabular}{|c|c|c|c|c|c|c|c|c|c|}
\hline \multirow{2}{*}{\multicolumn{2}{|c|}{$\begin{array}{l}\text { Motivation } \\
\text { Opportunity }\end{array}$}} & \multicolumn{4}{|c|}{ No barrier (\%) } & \multicolumn{4}{|c|}{ Barrier (\%) } \\
\hline & & \multicolumn{2}{|c|}{ No barrier } & \multicolumn{2}{|c|}{ Barrier } & \multicolumn{2}{|c|}{ No barrier } & \multicolumn{2}{|c|}{ Barrier } \\
\hline & & $\mathrm{SF}^{1}$ & $B^{1}$ & $\mathrm{SF}^{1}$ & $\mathrm{~B}^{1}$ & $\mathrm{SF}^{1}$ & $B^{1}$ & $\mathrm{SF}^{1}$ & $B^{1}$ \\
\hline \multirow[t]{2}{*}{ Ability } & No barrier & 19 & 27 & 21 & 14 & 7 & 7 & 5 & 3 \\
\hline & Barrier & 10 & 14 & 20 & 18 & 6 & 7 & 12 & 10 \\
\hline
\end{tabular}

1SF = snack foods, $B=$ beverages

The distribution across the profiles was similar for snack foods and beverages. The majority was motivated to a degree $(70 \%$ and $73 \%)$ and about half of the participants perceived to have sufficient ability (51\% and $50 \%$ ) to select low-calorie snacks. The profiles did however differ regarding opportunity; for snacks foods $41 \%$ of participants perceived the opportunity to be sufficient, while this was $54 \%$ for beverages. Correspondingly, the group with no barriers was larger for beverages (27\%) than for snack foods (19\%).

Identification of dominant motivation-opportunity-ability profiles

After considering the Calinsky-Harabasz, silhouette and Gap indices and investigating the patterns of significant and meaningful differences in terms of motivation, ability and opportunity, we concluded that a three-cluster solution provided the most parsimonious grouping. Table 3.8 shows the scores on the MOA factors for snack foods and beverages for each of the profiles. The three snack food profiles and the three beverage profiles show very similar patterns. Therefore they will be described jointly in the next paragraphs.

The 'no-barrier profile' is characterized by a very high motivation to choose lowcalorie over high-calorie snacks, without social pressure to do so. People with this profile perceive themselves as sufficiently able to make low-calorie over high-calorie 
choices and they also feel that the opportunity to make these choices is present. Overall, people with this profile report having no barriers to choose low-calorie over high-calorie snacks in real life. For both snack foods and beverages, the no-barrier profile has the highest overall proportion of low-calorie choices (69\% for snack foods and $76 \%$ for beverages).

The 'lack-of-opportunity profile' is also characterized by a high motivation to choose low-calorie over high-calorie snacks, coupled with some social pressure to do so. In contrast to the no-barrier profile, people with this profile perceive that there is insufficient opportunity to choose low-calorie snacks, particularly regarding the financial costs of obtaining low-calorie products. Even though they are motivated, people with the lack-of-opportunity profile are unsure about having sufficient knowledge and skills to be able to choose low-calorie over high-calorie snacks. The overall proportion of low-calorie choices is lower than in the no-barrier profile, both for snack foods (62\%) and beverages (70\%).

The 'lack-of-motivation profile' distinguishes itself from the no-barrier and lack-ofopportunity profile by a low motivation to choose low-calorie over high-calorie snacks. Although people with this profile think the opportunity to choose low-calorie over high-calorie snacks is less than those with the no-barrier profile, they do feel that it is more sufficient than people with the lack-of-opportunity profile. In addition to a low motivation score, the perceived ability to choose low-calorie over highcalorie options of those with the lack-of-motivation profile is also below average. In terms of snacking behaviour, the lack-of-motivation profile is characterized by the lowest overall proportion of low-calorie choices, both for snack foods (53\%) and beverages (61\%).

Table 3.9 further describes the profiles in terms of snacking and eating behaviours, and also provides socio-demographic characteristics of each profile. For both snack foods and beverages, the composition of the profiles differed significantly on gender, age, education and BMI. For the no-barrier profile we found an overrepresentation of females and higher educated people. The group with a lack-of-opportunity profile was quite similar to the overall respondent population in terms of gender, age and education level. However, compared with those with other profiles, relatively many people with the lack-of-opportunity profile were obese. Respondents with a lack-ofmotivation profile were more likely to be male, young and less educated. Daily breakfast and lunch consumption was highest for the no-barrier profile, followed by the lack-of-opportunity profile and the lack-of-motivation profile. 
Of the 1318 participants, the vast majority (69\%) was classified into the equivalent profiles for both snack categories, meaning that for them patterns of motivation, perceived opportunity and perceived ability were similar for snack foods and beverages. 


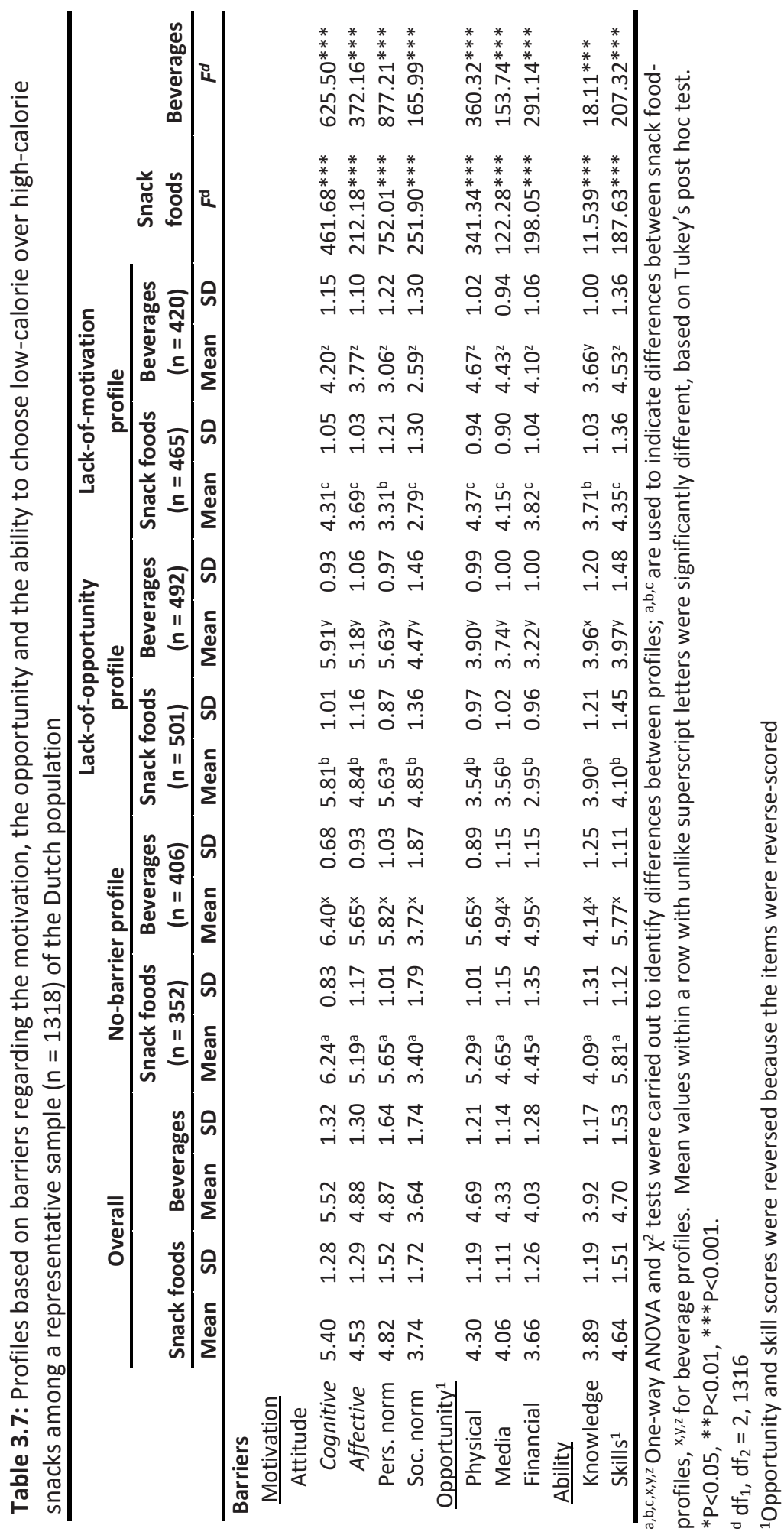




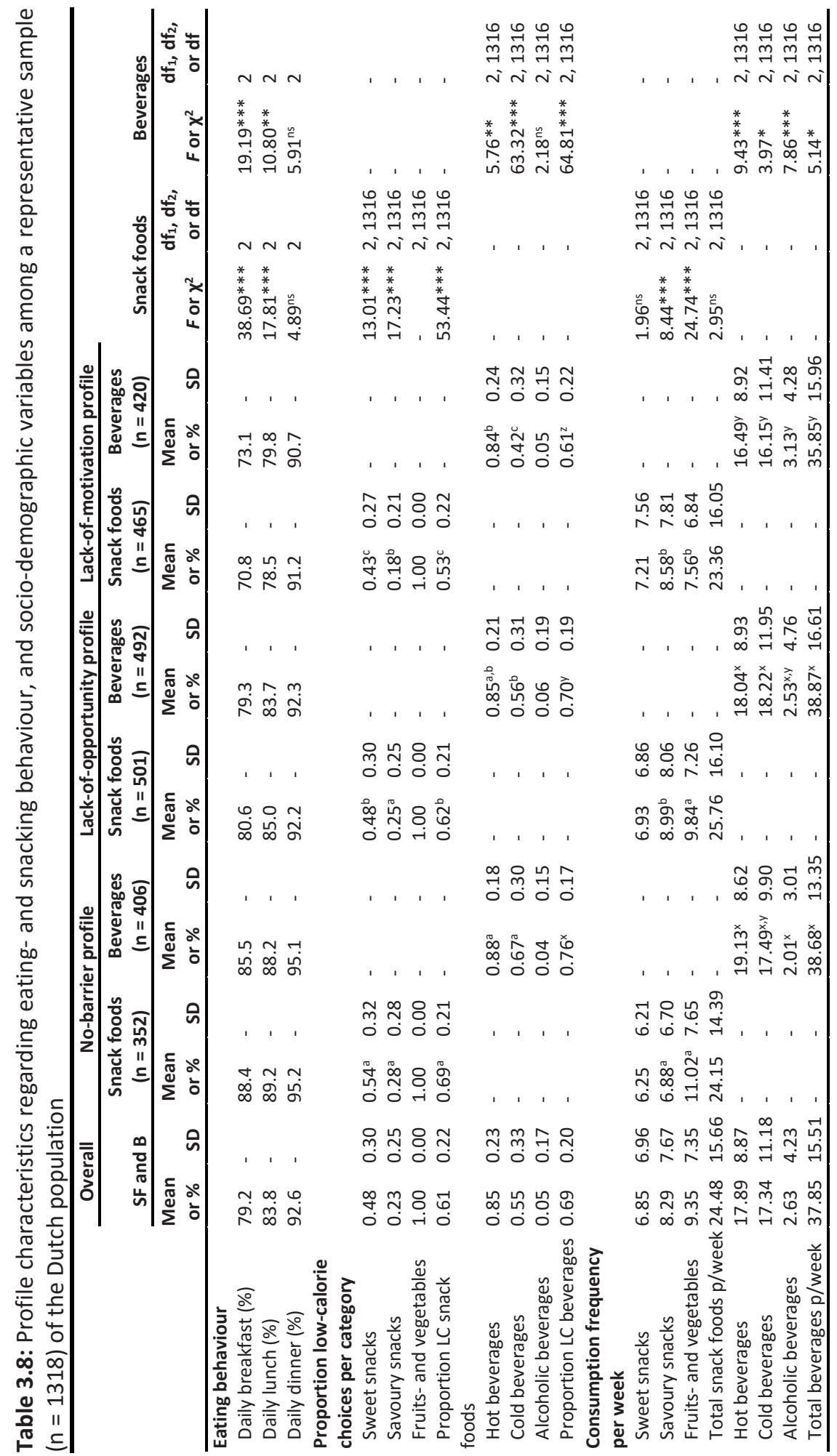

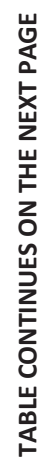




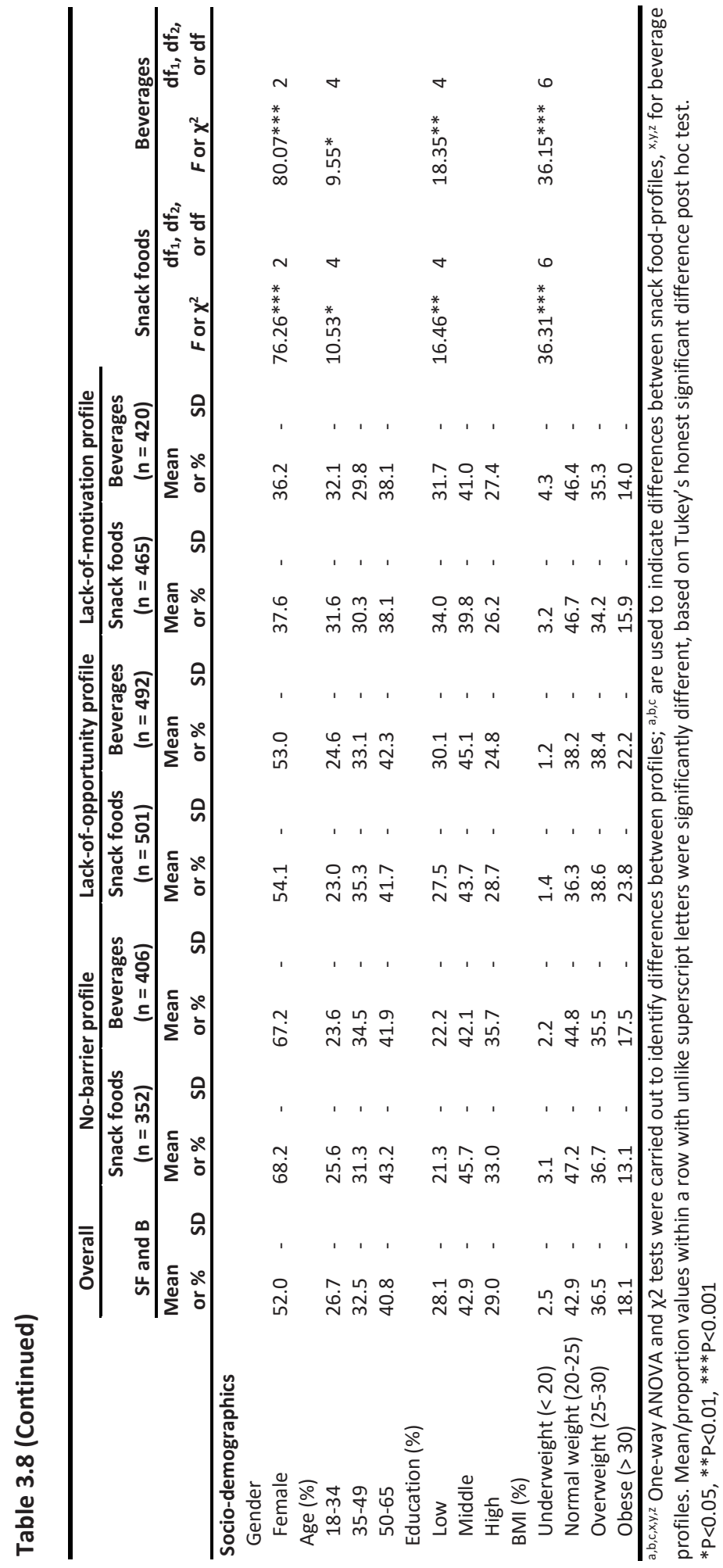


Relationship between motivation-opportunity-ability profiles and the log odds of choosing low-calorie snacks

To investigate the extent to which the dominant profiles are associated with lowcalorie snack choices compared with the MOA factors, we also performed a linear regression analysis in which the MOA factors in Step 2 were substituted for two dummy variables that represented the three dominant profiles. For snack foods, the three identified profiles accounted for $6.6 \%$ (adjusted $6.5 \%$ ) of the variance in the log odds of choosing low-calorie snack foods, compared with $13.2 \%$ (adjusted $12.6 \%$ ) for the MOA factors. Thus roughly half of the variance in the log odds of choosing low-calorie snack foods that could be explained by the MOA factors was covered by the identified three dominant MOA profiles.

For beverages, the three identified profiles accounted for $7.5 \%$ (adjusted $7.4 \%$ ) of the variance in the log odds choosing low-calorie beverages, compared with $10.1 \%$ (adjusted 9.5\%) for the MOA factors. Thus roughly three-quarters of the variance in the log odds of choosing low-calorie beverages that could be explained by the MOA factors was covered by the identified profiles. This means that, in light of implementing effective public health policies, splitting up the population into the three dominant profiles still captures a substantial part of the variance compared with the individual MOA factors.

When using the eight MOA snack food profiles that were constructed by means of a score split, explained variance in the log odds of choosing low-calorie snack foods was similar to the three dominant profiles: $6.8 \%$ (adjusted 6.3\%). For the eight MOA beverage profiles, explained variance in the log odds of choosing low-calorie beverages was lower than for the three dominant profiles: 6.3\% (adjusted 5.8\%). For tailoring intervention strategies to specific patterns of barriers, using the three dominant profiles thus is preferred over using the eight profile types suggested by the MOA framework.

\subsection{Discussion}

The present study employed Rothschild's MOA framework (1999) and examined the extent to which heterogeneity in barriers regarding the motivation, the perceived opportunity and the perceived ability to choose low-calorie over high-calorie snacks is associated with low-calorie snack choices in real life. The barriers were assessed by means of a survey, together with people's habitual snack consumption, which was 
measured via the FFQ. To explore generalizability across snack categories, barriers and habitual snack consumption were assessed separately for snack foods and beverages.

The results show that, both for snack foods and beverages, heterogeneity in barriers regarding the motivation, perceived opportunity and perceived ability to choose low-calorie over high-calorie snacks is associated with the proportion of low-calorie snack choices. Recognition of this heterogeneity in barriers adds to the predictive validity for low-calorie snack choices over and above the variance explained by sociodemographic variables. This shows that the MOA framework is a useful and meaningful tool for categorizing barriers for choosing low-calorie over high-calorie snacks. By further distinguishing between the concepts of cognitive and affective attitude, and personal and social norms as aspects of motivation; financial, physical and media aspects of opportunity; and knowledge and skills as aspects of ability, the present study also provides a blueprint to measure these barriers by developing new scales where existing scales were lacking.

In line with previous research on dietary behaviour (Dibsdall, Lambert, Bobbin, \& Frewer, 2003; Hearty, McCarthy, Kearney, \& Gibney, 2007), motivation was most strongly associated with low-calorie choices. This confirms that motivation is indispensable when it comes to maintaining and changing snacking behaviour. of particular importance is a positive affective attitude and strong personal norms regarding low-calorie snacking behaviour, which has been echoed in other studies (Paisley \& Sparks, 1998). Cognitive attitude came out as less influential, which is presumably due to a high level of consensus on the functional advantages of lowcalorie snacking over high-calorie snacking. This implies that to increase people's motivation to choose low-calorie snacks, it is more important to stress hedonic rather than utilitarian advantages of choosing low-calorie over high-calorie snacks.

A discrepancy between the costs of obtaining high-calorie and low-calorie foods has been repeatedly identified as a key contributor to the obesity problem (Drewnowski \& Darmon, 2005; Drewnowski \& Specter, 2004; Giskes et al., 2011; Kirk et al., 2010). The present study replicates this by finding that a lack of perceived financial opportunity to choose low-calorie over high-calorie snack foods is associated with lower proportions of low-calorie snack food choices. For beverages no such discrepancy was found, which is likely caused by the high availability of particular cheap low-calorie beverages such as water, tea and coffee. 
Perceptions of being able to easily interpret calorie information on snack food packaging increased the proportion of low-calorie snack food choices. Previous research indeed found that some people struggle with extracting calorie information from packages, resulting in less understanding of the caloric content of foods and beverages (Bos et al., 2013). For beverages, it is plausible that no effect of perceived ability on proportion low-calorie choices was found because of the high consumption of water, tea and coffee. When these beverages were removed from analysis, a borderline significant effect of being able to interpret calorie information on beverage packaging on the proportion of low-calorie beverage choices was found. These findings illustrate a widely echoed need for easy-to-interpret information and labelling on packaging to ameliorate ability (Grunert \& Wills, 2007; Magnusson, 2010).

Overall, barriers regarding the motivation, the perceived opportunity and the perceived ability to choose low-calorie over high-calorie snacks are moderately associated with proportions of low-calorie snack choices at the aggregate level across snack foods and across beverages. When specifically looking at regular and light soft drink choices, for which the only difference is the caloric value, the association between barriers and low-calorie choices is much stronger. This suggests that the origin of the moderate association between barriers and the overall proportion of low-calorie snacks primarily is caused by snack characteristics other than caloric value (e.g. taste). A choice for a low-calorie product (e.g. water or coffee) therefore does not necessarily reflect a preference for low-calorie beverages, as is the case when choosing for a light soft drink instead of a regular soft drink.

The present study identified three barrier profiles as dominant in the population: the no-barrier profile, the lack-of-opportunity profile and the lack-of-motivation profile. The identification of subgroups with similar barrier characteristics is beneficial for the effectiveness of interventions (Gordon et al., 2006) as, from a public health perspective, tailoring interventions to each individual is infeasible. The dominant profiles were close to identical across the snack foods and beverages contexts, thereby implying that with regard to diminishing barriers, similar intervention strategies can be implemented to stimulate low-calorie choices. Following the MOA framework's rationale, people with a no-barrier profile are best served by educational interventions that maintain their ability. People with a lack-ofopportunity profile will benefit most from marketing interventions that increase opportunity. People with a lack-of-motivation profile will be most likely to change their snacking behaviour when a combination of all three intervention strategies is 
implemented. Future research should investigate whether tailored intervention strategies are truly more effective than non-tailored interventions.

Confirming the inconclusiveness from earlier research (Gregori et al., 2011; Johnson \& Anderson, 2010), no relationship was found between the proportion of low-calorie snack choices and BMI. We did find that people with high proportions of high-calorie snack choices skipped breakfast and lunch more, thereby indicating that a lower caloric intake through meals is compensated with a higher caloric intake through snacks. Another potential confounder for which we did not control is physical activity, which is known to be of influence on the relationship between weight status and eating behaviour (Duval et al., 2008). Future research is encouraged to take the different definitions of snacking into account and ideally controls for a broader variety of factors that directly or indirectly influence weight status.

There are some limitations that apply to the present study. For measuring motivation, perceived opportunity and perceived ability, validated measures were used as much as possible, and when these were not available new scales were constructed based on earlier qualitative research (Bos et al., 2013). The results show that for the majority of measures internal consistency and fit were adequate. There were some issues with two oppositely phrased items for the Opportunity measure, however, and therefore they were removed from further analysis. We do think that for assessing Opportunity on a general level, we captured a sufficient part of the construct with the remaining items. Also, for the media measure within Opportunity we used only two items. Future research on barriers for food choices therefore requires further expansion and refinement of these measures.

The behavioural determinants in Rothschild's MOA framework consist of actual motivations, opportunities and abilities. For the present study, however, we used perceptions of opportunities and abilities regarding low-calorie snacking behaviour. This has both practical and theoretical reasons. The practical reason is that it would be burdensome to measure actual opportunity, because it would involve the continuous mapping of different aspects of the food-choice environment (e.g. the amount of advertising, the ratio of low-calorie and high-calorie products). The theoretical reason is that it is plausible that for acceptance of intervention strategies, perceptions of barriers are more important than actual barriers (Bos et al., 2013). When people do not accept interventions, the risk of reactance towards the intended behaviour increases (Brehm, 1966). When people do accept interventions, however, rationalization of behaviour likely occurs (Laurin et al., 2012). Where 
reactance will impair intervention effectiveness, rationalization will greatly benefit it.

As is argued in literature on dietary behaviour, food choices are multifaceted because they depend on situational, dynamic and complex factors, such as time constraints and social influence (Sobal \& Bisogni, 2009). The present study applied a framework that encompasses both individual and environment influences on foodchoice behaviour and examined these barriers on a more aggregated level. For future research, the results of the present study can be used as a starting point for identifying which barriers are perceived as most prevalent in different food-choice situations.

In the present study, we used an FFQ as it is particularly suitable for eliciting habitual food consumption (Cade et al., 2002). However, although the questionnaire is widely applied, there are concerns about under-reporting of consumption frequencies (Bingham et al., 2003). For the present study this concern may be less of a problem, as under-reporting of both low-calorie and high-calorie snack consumption frequencies does little to change the proportion of low-calorie choices. Furthermore, FFQ are seen as adequately valid dietary assessment measures for aetiological studies (Byers, 2001).

In addition, our classification of the FFQ items into low-calorie and high-calorie choices is somewhat arbitrary. We used a threshold of 418.4kJ (100kcal) per portion, so that snacks that are associated with obesity (crisps, cookies, sugar-sweetened beverages) (Cohen et al., 2010) were categorized as high-calorie snacks, and fruits and vegetables were categorized as low-calorie snacks. As a result, individual differences regarding portion sizes were not taken into account, which could partially explain the lack of relationship between weight status and snacking behaviour. The employed classification method is, however, similar to caloric content classification methods in other food-choice research (Bandini et al., 1999; Kocken et al., 2015).

In the classification of snacks, the present study emphasized their caloric values (lowcalorie vs. high-calorie) because an imbalance between energy intake and energy expenditure is the main driver of overweight and obesity (Bleich et al., 2008). In light of responsible snacking choice behaviour, other characteristics than the caloric value of snacks can also be relevant. Snacks with a high nutritional value, for instance, can contribute to a varied and healthy overall dietary pattern. Research on the 
generalizability of the results to other product characteristics would therefore be valuable.

\subsection{Conclusions}

Employing Rothschild's MOA framework, the present study contributes to the understanding of unhealthy and irresponsible food-choice behaviour by demonstrating that barriers within motivation, perceived opportunity and perceived ability to choose low-calorie snacks are related to real-life snacking behaviour. In addition, the identification of three dominant barrier profiles (the no-barrier profile, the lack-of-opportunity profile and the lack-of-motivation profile) provides a strong case for the tailoring of intervention strategies.

Additional research is still needed to examine whether tailoring interventions to barrier profiles does indeed increase the acceptance and subsequently the effectiveness of interventions. Our findings furthermore suggest that, even though measures were carefully selected, improvements in the measurement of barriers for food choice are needed. 

CONSUMER ACCEPTANCE OF POPULATION-LEVEL INTERVENTION STRATEGIES FOR HEALTHY FOOD

CHOICES

\section{The role of perceived effectiveness and perceived fairness}

This chapter is published as:

Bos, C., Van der Lans, I., Van Rijnsoever, F., Van Trijp, H.C.M. (2015) Consumer acceptance of population-level intervention strategies for healthy food choices: the role of perceived effectiveness and perceived fairness 


\section{Abstract}

\section{Background}

The present study investigates acceptance of intervention strategies for low-calorie snack choices that vary regarding the effect they have on consumers' freedom of choice (providing information, guiding choice through (dis)incentives, and restricting choice). We examine the mediating effects of perceived effectiveness and perceived fairness, and the moderating effects of barriers to choose low-calorie snacks and perceived responsibility for food choice.

\section{Methods}

Data was collected through an online survey, involving three waves that were completed over a seven week timespan. Information was collected on barriers and perceived responsibility, and evaluations of a total of 128 intervention strategies with varying levels of intrusiveness that were further systematically varied in terms of source, location, approach/avoidance, type, and severity. A total of 1173 respondents completed all three waves.

\section{Results}

We found that the effect of intervention intrusiveness on acceptance was mediated by the perceived personal- and societal effectiveness, and the perceived fairness of interventions. For barriers and perceived responsibility, only main effects on intervention-specific beliefs were found. Government interventions were accepted less than interventions by food manufacturers.

\section{Conclusions}

The present study shows that acceptance of interventions depends on perceptions of personal- and societal effectiveness and fairness, thereby providing novel starting points for increasing acceptance of both existing and new food choice interventions. 


\subsection{Introduction}

Systematic caloric overconsumption is the main driver of increasing overweight and obesity rates in the Western world (Shelley, 2012; Swinburn et al., 2011). In the United States caloric intake through meals increased 8\% from 1977 to 2006, while intake through snacks more than doubled (130\%) (Popkin \& Duffey, 2010). Additionally, in Europe this trend has been detected (Gracia \& Albisu, 2001; Ovaskainen et al., 2005). Snacking, therefore, is believed to be an important contributor to the obesity problem. Since the foods and drinks that are consumed between meals are generally high in calories (Zizza et al., 2001), stimulating people to choose low-calorie over high-calorie snacks can be an effective method to reduce overweight and obesity rates (Cohen et al., 2010).

Policy makers, scientific researchers, and health-care workers have become increasingly interested in intervention strategies that stimulate healthier and more responsible food-choice behaviour (Gortmaker et al., 2011), particularly because consuming high quantities of calories through sugar and fat potentially becomes addictive (Avena, Rada, \& Hoebel, 2008; Meule \& Gearhardt, 2014). These intervention strategies, when effective, can reduce the burden of obesity and related non-communicable diseases such as diabetes, cancers, and cardiovascular diseases (Traill et al., 2010). Intervention strategies come in many shapes and forms, varying from public health campaigns to a ban of advertising unhealthy foods to children.

The effectiveness of intervention strategies for healthy eating, however, leaves much to be desired. An extensive analysis of the effectiveness of healthy eating policies in Europe shows that the behavioural impact of different types of interventions (e.g., public information campaigns and fiscal measures) is inconclusive, at best (Capacci et al., 2012). Even healthy eating interventions aimed at specific demographic target groups (e.g., children and the overweight) are known to have only little effect on behaviour (Brambila-Macias et al., 2011; Kesten et al., 2011; Maes et al., 2012; Paul-Ebhohimhen \& Avenell, 2008).

To increase effectiveness, scholars stress that intervention strategies need to be accepted by those who are affected by them (Diepeveen et al., 2013; Kelly et al., 2009). The level of acceptance affects the degree to which people are prepared to change their behaviour. Reactance theory (Brehm, 1966) suggests that insufficient acceptance of interventions leads people to be less likely to perform the desired behaviour. In contrast, sufficient acceptance elicits rationalization, leading people to 
be more likely to adopt the intended behaviour (Laurin et al., 2012). In addition, acceptance of interventions is an important condition for its implementation because stakeholders will be reluctant to intervene without public support (Schuitema et al., 2011). Despite its importance, extensive knowledge on the processes underlying acceptance of intervention strategies for food choices is currently lacking (Mazzocchi et al., 2014).

The aim of the present study is to investigate the processes underlying acceptance of intervention strategies for choosing low-calorie over high-calorie snacks. Furthermore, we examine differences in acceptance of interventions that vary regarding the effect they have on consumers' individual freedom of choice (level of intrusiveness). Hereto, we use Nuffield's intervention ladder (2007), which distinguishes between providing information (low intrusiveness), guiding choice by providing incentives or disincentives (medium intrusiveness), and restricting choice (high intrusiveness). In addition, to explore whether the processes underlying acceptance are robust across snack categories, we distinguish between beverageand snack food interventions. The following section describes the theoretical background and the hypotheses of the study.

\subsection{Theoretical Framework and Hypotheses}

Research on processes underlying the acceptance of interventions has shown that two intervention-specific beliefs are particularly important for acceptance: the perceived effectiveness and the perceived fairness (Eriksson et al., 2008). The perceived effectiveness refers to whether people think interventions will be successful in stimulating a particular behaviour, thereby differentiating between effectiveness for themselves and for society as a whole. The perceived fairness, on the other hand, refers to the extent to which people perceive interventions to be a fair way of stimulating a particular behaviour. This research, however, originates from the transport policy domain and it is, therefore, unclear whether the results generalize to intervention acceptance in other domains.

A qualitative study from Bos et al. (2013) suggests that also within the food-related domain perceptions of effectiveness and fairness positively influence acceptance of interventions. They found that when people were asked to elaborate on why they accepted some interventions more than others, they often stated reasons that 
involved the personal- and societal effectiveness, and fairness of interventions. Therefore, we hypothesize that:

Hypothesis 1a: There is a positive effect of perceived personal effectiveness on the acceptance of snack choice interventions.

Hypothesis 1b: There is a positive effect of perceived societal effectiveness on the acceptance of snack choice interventions.

Hypothesis 1c: There is a positive effect of perceived fairness on the acceptance of snack choice interventions.

Literature on acceptance further suggests that the effect on one's individual freedom of choice is a strong predictor of intervention acceptance. A comprehensive review by Diepeveen et al. (2013) concludes that acceptance of government interventions to change different health-related behaviours, including food-related behaviour, is strongly influenced by their level of intrusiveness. Using Nuffield's intervention ladder (2007), the review demonstrates that less intrusive interventions, of which the main purpose is to provide information, are generally accepted more than intrusive interventions, which mainly aim to restrict choices. This effect of intrusiveness has also been found in choice experiments; Pechey et al. (2014) conclude that for reducing alcohol intake the least intrusive interventions are most accepted. We therefore hypothesize that:

Hypothesis 2a: More intrusive snack choice interventions are less accepted.

Since literature suggests that acceptance is mediated by the perceived effectiveness and perceived fairness (Bos et al., 2013; Eriksson et al., 2008), one would expect an effect of intrusiveness on these intervention-specific beliefs as well. However, in the literature on food-related policies, this effect has received surprisingly limited research attention. To our knowledge, there are only a few studies that relate perceptions of intervention effectiveness and intrusiveness (Pechey et al., 2014; Promberger et al., 2012). These studies, however, present people with information about the expected effectiveness of interventions and they do not ask people about their perceptions of intervention effectiveness. Therefore, no conclusions can be drawn about the relation between intervention intrusiveness and perceived effectiveness. 
In the transport policy context, Eriksson et al. (2008) found that improving public transport was perceived as more effective than raising taxes on fossil fuels. As raising taxes on fossil fuels obviously is more intrusive than improving public transport, this suggests that there is a relation between intrusiveness and perceptions of effectiveness. To examine whether this relation holds for snack choice interventions, we hypothesize that:

Hypothesis $\mathbf{2 b}$ : More intrusive snack choice interventions are perceived to be less effective on a personal level.

Hypothesis 2c: More intrusive snack choice interventions are perceived to be less effective on a societal level.

In addition, Eriksson et al. (2008) found that improving public transport was also perceived as fairer than raising taxes on fossil fuels. Within the food-related policy context, Promberger et al. (2011) examined the perceived fairness of financial interventions versus medical interventions. These types of interventions, however, are not easily categorized in terms of level of intrusiveness and therefore the relation between intervention intrusiveness and perceived fairness in a food-related policy context remains unclear. Based on the research in the transport policy domain, we hypothesize that:

Hypothesis 2d: More intrusive snack choice interventions are perceived to be less fair.

Connecting the hypotheses about the effect of intrusiveness on perceptions of effectiveness and fairness ( $2 a, 2 b, 2 c$, and $2 d$ ) with the hypotheses about the positive effect of the perceived effectiveness and the perceived fairness on acceptance ( $1 \mathrm{a}$, $1 \mathrm{~b}$, and $1 \mathrm{c})$, we come to the overarching hypothesis that:

Hypothesis 3: The effect of intrusiveness on acceptance of interventions is mediated by the perceived personal- and societal effectiveness, and the perceived fairness of interventions.

With respect to effectiveness of interventions, Rothschild's Motivation-OpportunityAbility (MOA) framework (1999) theorizes that it depends on people's barriers whether an intervention will be effective: people who lack ability need education (equivalent to providing information), people who lack opportunity need marketing 
(equivalent to guiding choice), and people who lack motivation need law (equivalent to restricting choice). Extrapolating this logic to the present study, we expect that people's perceived barriers for choosing low-calorie over high-calorie snacks influences the relation between intervention intrusiveness and perceived effectiveness.

Bos, Van der Lans, Van Rijnsoever, and Van Trijp (2015b) found three types of barrier profiles for choosing low-calorie snacks over high-calorie snacks: the no-barrier profile, which is characterized by high levels of motivation, opportunity, and ability; the lack-of-opportunity profile, which has a high level of motivation, a low level of opportunity, and a medium level of ability; and the lack-of-motivation profile, which features a low level of motivation and medium levels of opportunity and ability. These profiles applied to both the beverage and snack food categories. Reasoning from the MOA framework, we hypothesize that:

Hypothesis 4a: The relation between intervention intrusiveness and the perceived personal effectiveness of interventions is moderated by perceived barriers for choosing low-calorie snacks.

Hypothesis 4b: The relation between intervention intrusiveness and the perceived societal effectiveness of interventions is moderated by perceived barriers for choosing low-calorie snacks.

Additionally, we expect that the relation between intervention intrusiveness and perceptions of fairness will be moderated as well. Studies suggest that one's perception about the responsibility for food choices is related to the perceived fairness of interventions (Lusk \& Ellison, 2013; Pearl \& Lebowitz, 2014). These studies found that the more one believes that responsibility for food choices lie with other stakeholders such as the government and food suppliers, the more they thought interventions to change the food environment were fair. When one felt that the responsibility only lies with people themselves, however, support for more intrusive interventions was low. We therefore hypothesize that:

Hypothesis 5: The relation between intervention intrusiveness and the perceived fairness of interventions is moderated by people's perceptions of personal responsibility for food choices. 
By testing the conceptual framework that is depicted in figure 4.1, we aim to better understand the processes that underlie acceptance of intervention strategies for low-calorie snack choices as well as the influence of intervention intrusiveness on these processes. With this knowledge policy makers in the food domain can anticipate negative and positive evaluations of new intervention strategies and address these sentiments accordingly.

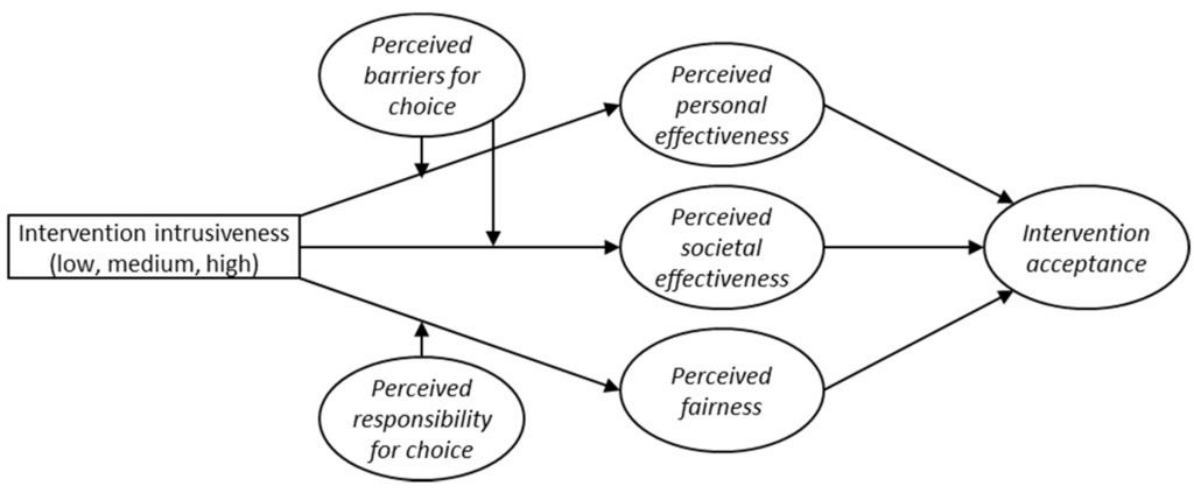

Figure 4.1: Overview of the present study's conceptual framework.

\subsection{Material and Methods}

\section{Procedure and Sample}

The research protocol for the project "Matching supply and demand side preferences in food innovation" (NWO project number 2100882000), which includes the present article, was submitted to the Social Science Ethics Committee of the Wageningen University and subsequently approved for fulfilling the Wageningen University code of conduct.

An online survey was conducted, comprising of three waves. Wave 1 was divided into two parts, which were presented in a random order: one part focused on individuals' motivation, opportunity, and ability to choose low-calorie over highcalorie beverages, while the other part focused on the same factors for snack foods. Four weeks later (Wave 2) respondents filled out a Food Frequency Questionnaire (not relevant for the present study) and a questionnaire regarding the perceived responsibility for food choices. Another three weeks later (Wave 3) respondents were asked to rate 16 intervention strategies for stimulating low-calorie snack 
choices on perceived effectiveness, perceived fairness and acceptance. Respondents were assigned to evaluate either beverages or snack food interventions. Splitting up the data collection in three waves was done to prevent respondent burden and the intermissions were employed to minimize consistency bias.

Data was collected in the Netherlands by GfK (Gesellschaft für Konsumforschung), which is a commercial marketing research agency. A representative sample of the Dutch population based on gender, age, and education level was recruited from the GfK Online Panel. For their participation, respondents received a number of "GfK points" (equivalent to 5 euros), with which they could order coupons. Of the 1573 respondents that completed Wave 1, a total of 1318 completed Wave 2, and 1173 completed all three waves. Response rates were $70 \%, 84 \%$, and $89 \%$, respectively. Additionally, a new group of 146 respondents completed solely Wave 3, to analyse bias that may have occurred through the completion of Wave 1 and 2. A summary of characteristics of the 1319 respondents can be found in Table 4.1.

Table 4.1: Respondent characteristics

\begin{tabular}{lcccc}
\hline & \multicolumn{2}{c}{ Wave 1, 2, and 3 $(\boldsymbol{n}=\mathbf{1 1 7 3})$} & \multicolumn{2}{c}{ Only Wave 3 $(\boldsymbol{n}=\mathbf{1 4 6})$} \\
\cline { 2 - 5 } & $\begin{array}{c}\text { Males } \\
(\boldsymbol{n}=\mathbf{5 5 9})\end{array}$ & $\begin{array}{c}\text { Females } \\
(\boldsymbol{n}=\mathbf{6 1 4})\end{array}$ & $\begin{array}{c}\text { Males } \\
(\boldsymbol{n}=\mathbf{7 6})\end{array}$ & $\begin{array}{c}\text { Females } \\
(\boldsymbol{n}=\mathbf{7 0})\end{array}$ \\
\hline Age & & & & \\
18-34 & $25.6 \%$ & $27.4 \%$ & $27.6 \%$ & $28.6 \%$ \\
$35-49$ & $34.5 \%$ & $31.2 \%$ & $34.2 \%$ & $38.6 \%$ \\
50-65 & $39.9 \%$ & $41.4 \%$ & $38.2 \%$ & $32.9 \%$ \\
Education ${ }^{1}$ & & & & \\
Low & $26.3 \%$ & $29.3 \%$ & $25.0 \%$ & $27.1 \%$ \\
Middle & $41.7 \%$ & $43.8 \%$ & $46.1 \%$ & $40.0 \%$ \\
High & $32.0 \%$ & $26.9 \%$ & $28.9 \%$ & $32.9 \%$ \\
\hline
\end{tabular}

${ }_{1}^{1}$ Based on International Standard Classification of Education (ISCED): Low equates to lowersecondary education or less, middle equates to upper-secondary or post-secondary education, and high equates to tertiary education

\section{Stimuli}

The intervention strategies that respondents were confronted with in Wave 3 differed on the level of intrusiveness (providing information versus guiding choice versus restricting choice). For each level of intrusiveness, intervention strategies were operationalized in multiple ways to check whether our findings with respect to intrusiveness generalize across different implementations. Based on earlier research on acceptance of interventions in the food domain (Bos et al., 2013; Pechey et al., 2014), operationalizations varied with respect to the following characteristics: 1) the source of the intervention (the government versus a group of leading food 
manufacturers), 2) the location (supermarkets versus points-of-purchase on gas- and train stations, and 3 ) approach-avoidance (encouraging low-calorie choices versus discouraging high-calorie choices). Since restricting choice is logically incompatible with encouraging low-calorie-choices, this combination was not offered to respondents.

Intervention strategies were further varied in terms of intervention type and severity level (see Table 4.2). Providing information was divided into product labelling and information campaigns, guiding choice was divided into financial and assortment (dis)incentives, and restricting choice was divided into restriction of physical choice and restriction of advertising. Similarly, severity was nested with the type of intervention.

Table 4.2: Intervention type and severity level

\begin{tabular}{|c|c|}
\hline Type & Severity \\
\hline Product labelling & $\begin{array}{l}\text { Back-of-pack, } \\
\text { Front-of-pack }\end{array}$ \\
\hline Information campaigns & $\begin{array}{l}\text { On location, } \\
\text { On location + media }\end{array}$ \\
\hline Financial (dis)incentives & $\begin{array}{l}10 \%, 20 \% \\
30 \%, 40 \%\end{array}$ \\
\hline Assortment (dis)incentives & $\begin{array}{l}15 \%, 30 \%, \\
45 \%, 60 \%\end{array}$ \\
\hline Physical choice restriction ${ }^{1}$ & $\begin{array}{l}15 \%, 30 \%, \\
45 \%, 60 \%\end{array}$ \\
\hline Advertising restriction $^{1}$ & $\begin{array}{l}15 \%, 30 \%, \\
45 \%, 60 \%\end{array}$ \\
\hline
\end{tabular}

${ }^{1}$ Restriction of a \% of the most high-calorie snacks

The 128 possible intervention strategies (32 informing, 64 guiding, 32 restricting) were divided into eight blocks with each 16 interventions (four informing, eight guiding, and four restricting). Respondents were randomly assigned to one of the eight blocks. Each block was designed in such a way that within the three levels of intrusiveness, respondents were confronted with every attribute and each level. This way, the blocking factor was un-confounded with the main effects of intrusiveness level and intervention characteristics, as well as with the interaction effects of intrusiveness level and each of the intervention characteristics.

Respondents were presented with a description of the intervention strategy, which was coupled with corresponding icons that illustrated the intervention. The structure 
of the description was as follows: "Through policy, the government obliges supermarkets to extend shelf space for low-calorie snack foods with $30 \%$." Above this description, respondents saw icons that illustrated the attributes: a government logo, a supermarket icon, and a representation of shelf space extension with the number $30 \%$.

\section{Measures}

\section{Evaluations of intervention strategies}

Respondents evaluated a total of 16 intervention strategies on four items. These were seven-point Likert-scale items, with labels ranging from "completely disagree" to "completely agree" (coded from 1 to 7 for the analyses), and consisted of the perceived personal- and societal effectiveness (two items: "This strategy would lead me to choose low-calorie over high-calorie beverages/snack foods more often than I currently do" and "This strategy would lead a lot of people to choose low-calorie over high-calorie beverages/snack foods more often than they currently do), the perceived fairness (one item: "I think this strategy is a fair way to encourage people to choose low-calorie over high-calorie beverages/snack foods"), and the acceptance of the interventions (one item: "I support the implementation of this strategy").

\section{Barriers for low-calorie choices}

To map respondents' barriers we employed Rothschild's MOA framework (1999), which theorizes that both individual-level and environmental determinants drive food-choice behaviour. Because the MOA framework itself does not dictate how the Motivation, Opportunity, and Ability constructs should be measured, we used and adapted scales from existing scales from the behavioural theories. In total, 27 items were used for measuring Motivation (12), Opportunity (10), and Ability (5). A detailed description of the construction of the questionnaire can be found in Bos et al. (2015b).

\section{Perceived personal responsibility for food choice}

The measure for perceived personal responsibility for food choice was adapted from Lusk and Ellison (2013) and was assessed by one item: "To what extent do you think that the following stakeholders are (partly) responsible for whether people choose low-calorie or high-calorie snacks?" Respondents were asked to distribute 100 points over five stakeholders: the government, food manufacturers, food suppliers other than supermarkets (bakeries, restaurants), supermarkets, and consumers 
themselves. The final score consisted of the number of points that were allocated to "consumers themselves".

\section{Data analysis}

To explore whether there is a positive effect of the perceived personal- and societal effectiveness and the perceived fairness on the acceptance of interventions ( $\mathrm{H} 1)$, we regressed acceptance simultaneously on these three constructs by multiple linear regression in SPSS (Version 22) (IBM Corp., Armonk, NY, USA). For all the variables in this analysis we used respondents' mean-centred scores to cancel out individual differences in mean acceptance and to make it reasonable that the errors will be uncorrelated (Seltman, 2012).

To investigate the effect sizes of intrusiveness on perceptions of personal- and societal effectiveness, fairness, and acceptance ( $\mathrm{H} 2)$, and the moderating effects of barriers for low-calorie choices and the perceived personal responsibility for food choice (H4 and $\mathrm{H} 5$ ), we performed an analysis of (co)variance in SPSS. For this analysis we used respondents' mean-centred scores. We restricted our interpretation of results to only those (large enough) effects with a partial $\eta^{2}>0.015$.

To provide information on respondents' absolute evaluations of interventions, we report the means of the perceived personal- and societal effectiveness, the perceived fairness, and acceptance in terms of the original scale (not mean-centred) to interpret the direction of the effects. Within each analysis of variance, we ran post hoc comparisons using the Games-Howell procedure to control the family-wise error rate.

To explore whether the effect of intrusiveness on acceptance is mediated by the perceived personal- and societal effectiveness, and the perceived fairness (H3), we added these intervention-specific beliefs into the analysis of (co)variance. To analyse mediation effects, we followed the steps for testing mediational hypotheses, as presented by Baron and Kenny (1986).

To explore direct (between-subjects) effects of barriers on absolute perceptions of intervention effectiveness, we used original (not mean-centred) scores to identify differences between the three dominant barrier profiles regarding perceptions of effectiveness. Profiles of barriers were identified by means of cluster analysis. An extensive description of the data analysis and the barrier profiles that were identified can be found in Bos et al. (2015b). 
Additionally, we ran mixed model variants of the regression analysis and the analyses of (co)variance on the original (not mean-centred) data (using a random intercept). These mixed models are another way of controlling for possibly-correlated error terms due to multiple observations from each respondent. Within the mixed models, we used questionnaire version as a blocking factor, to rule out that this influenced the results. We found that the $p$-values for the mixed model analyses were very similar to the $p$-values of the mean-centred data analyses, and therefore did not lead to different conclusions. Questionnaire version did not have a significant effect in any of the mixed model analyses. For clarity purposes, we only report the results of the analyses with the mean-centred data.

\subsection{Results}

Prior to testing Hypothesis $1 a, 1 b$, and $1 c$, we closely inspected correlations between the perceived personal- and societal effectiveness, the perceived fairness, and acceptance of beverage- and snack food interventions (Table 4.3). There were positive correlations between perceived personal- and societal effectiveness and acceptance ( $r=0.57-0.63)$ and a strong positive correlation between perceived fairness and acceptance $(r=0.82)$. Correlations between perceived personal- and societal effectiveness and perceived fairness were also positive $(r=0.54-0.69)$. For the remainder of the results section we will use the overarching term "snack choice interventions" if results are similar for beverage- and snack food interventions.

Table 4.3: Correlations between intervention-specific beliefs and acceptance of beverage interventions (upper part) and snack food interventions (lower part).

\begin{tabular}{lllll}
\hline & $\begin{array}{c}\text { Personal } \\
\text { Effectiveness }\end{array}$ & $\begin{array}{c}\text { Societal } \\
\text { Effectiveness }\end{array}$ & Fairness & Acceptance \\
\hline Personal effectiveness & 1.00 & $0.69^{* * *}$ & $0.54^{* * *}$ & $0.57^{* * *}$ \\
Societal effectiveness & $0.67^{* * *}$ & 1.00 & $0.57^{* * *}$ & $0.59^{* * *}$ \\
Fairness & $0.55^{* * *}$ & $0.58^{* * *}$ & 1.00 & $0.82^{* * *}$ \\
Acceptance & $0.59^{* * *}$ & $0.63^{* * *}$ & $0.82^{* * *}$ & 1.00 \\
\hline$* * * p<0.001$ & & & &
\end{tabular}

Subsequently, a multiple linear regression analysis of acceptance on the perceived personal- and societal effectiveness, and the perceived fairness of snack choice interventions was performed. Variance Inflation Factor (VIF) values were <3, 
indicating that multi-collinearity did not pose a problem. The intervention-specific beliefs accounted for $71 \%$ of the variance in acceptance of snack choice interventions. Perceptions of personal- $(\beta=0.115)$ and societal effectiveness $(\beta=0.144)$, and fairness $(\beta=0.679)$ had a significant positive effect on acceptance of interventions. This indicates that there was a substantial individual effect of each intervention-specific belief on acceptance, even though they were positively correlated. We, therefore, accept Hypothesis $1 a, 1 b$, and $1 c$ and conclude that there is a positive effect of the perceived personal- and societal effectiveness, and the perceived fairness on the acceptance of interventions that stimulate low-calorie snack choices.

Table 4.4 shows the results from analyses of variance of intrusiveness and intervention attributes on the perceived personal- and societal effectiveness, the perceived fairness, and acceptance of snack choice interventions. To check whether completing Waves 1 and 2 influenced Wave 3 evaluations, we compared the effects of intrusiveness on intervention-specific beliefs and acceptance with the same effects in the new group of respondents $(n=146)$. Only for perceived fairness a significant difference $(p=0.025)$ was found. The effect size, however, was negligibly low (partial $\eta^{2}=0.001$ ). The results indicate that the level of intrusiveness influenced both the perceived fairness and acceptance (partial $\eta 2=0.016-0.030$ ). In contrast, the level of intrusiveness did not influence perceptions of personal- and societal effectiveness (partial $\eta^{2}=0.000-0.002$ ); therefore we reject Hypothesis $2 b$ and $2 c$. 


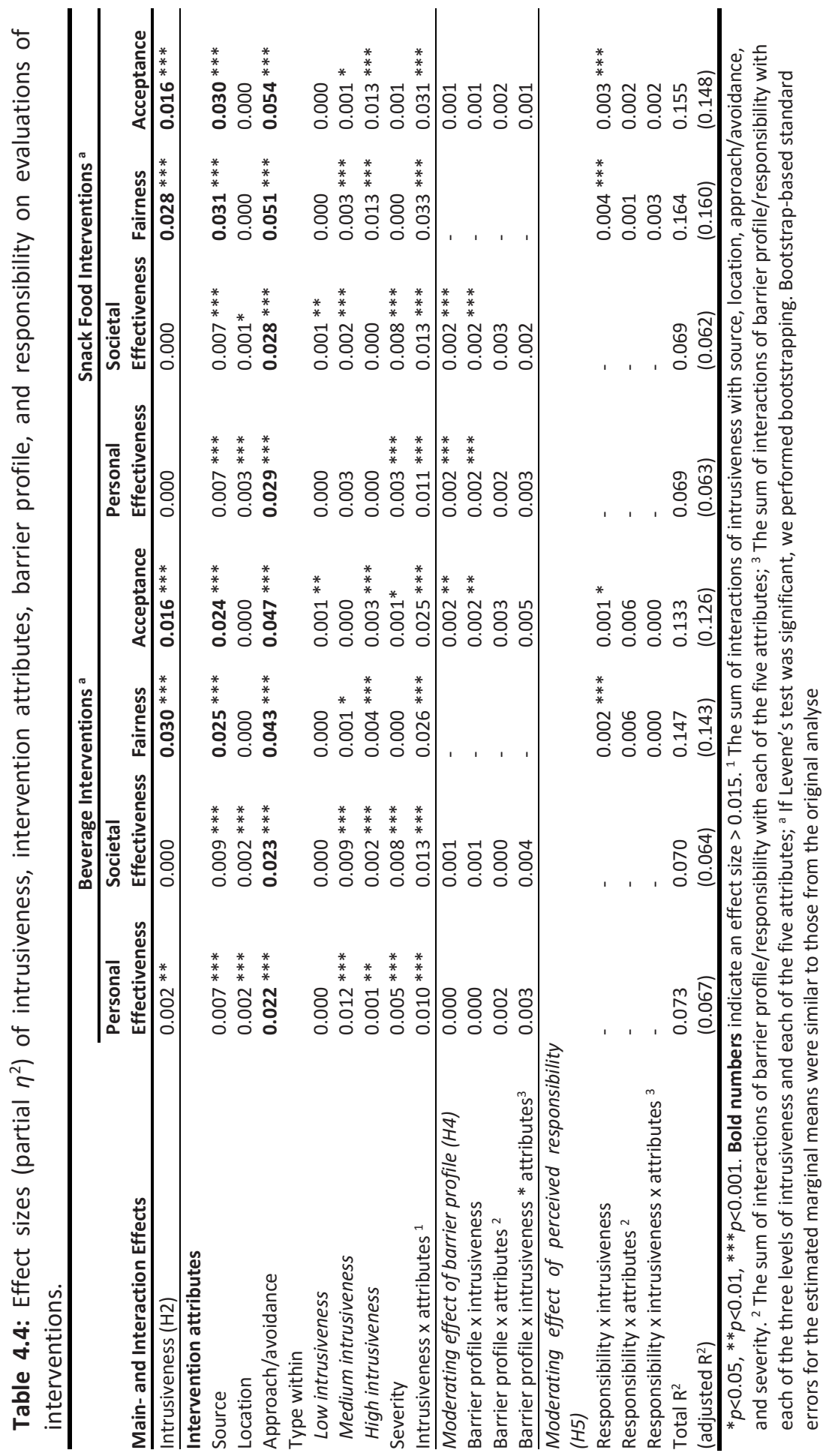


The directions of the main effects of intrusiveness on the perceived fairness and acceptance can be derived from Table 4.5 (beverage interventions) and Table 4.6 (snack food interventions). Hypothesis 2 a is confirmed, because more intrusive snack choice interventions are indeed less accepted. Similarly, more intrusive interventions are perceived as less fair, thereby confirming Hypothesis $2 \mathrm{~d}$.

With respect to intervention attributes, we see in Table 4.4 that location, type, and severity either have no or a negligibly small effect on the evaluations of interventions (partial $\eta^{2}=0.000-0.013$ ). For source (partial $\eta^{2}=0.007-0.031$ ) and approach/avoidance (partial $\eta^{2}=0.022-0.054$ ), however, evaluations of their respective levels were different. The directions of the main effects of source, approach/avoidance, and the interactions of intrusiveness with source and approach/avoidance can be found in Table 4.5 and Table 4.6.

We did not develop specific hypotheses about different effects of attributes on evaluations of interventions. Still, we found that interventions that encourage lowcalorie choices were perceived as more effective, fairer, and more acceptable than those that discourage high-calorie choices. This only applied to interventions that guide choice through (dis)incentives; for interventions that provide information, encouragement, and discouragement were evaluated similarly. As restricting choice only applies to discouraging high-calorie choices, no effects can be reported.

A similar pattern emerged for source: interventions implemented by the government were perceived as less fair and were also less accepted than interventions implemented by a group of leading food manufacturers. This was true for interventions that guide choice and interventions that restrict choice. Again, for providing information evaluations were similar for both sources. 
Table 4.5: Means of the effect sizes (partial $\eta^{2}>0.015$ ) for beverage interventions.

\begin{tabular}{|c|c|c|c|c|c|c|c|c|}
\hline \multirow{2}{*}{$\begin{array}{l}\text { Main- and Interaction } \\
\text { Effects }\end{array}$} & \multicolumn{2}{|c|}{$\begin{array}{c}\text { Personal } \\
\text { Effectiveness }\end{array}$} & \multicolumn{2}{|c|}{$\begin{array}{c}\text { Societal } \\
\text { Effectiveness }\end{array}$} & \multicolumn{2}{|c|}{ Fairness } & \multicolumn{2}{|c|}{ Acceptance } \\
\hline & Mean & SD & Mean & SD & Mean & SD & Mean & SD \\
\hline \multicolumn{9}{|l|}{$\begin{array}{l}\text { Intrusiveness and } \\
\text { Intrusiveness } x \text { Type }\end{array}$} \\
\hline Inform choice (low) & $4.12^{\mathrm{y}}$ & 1.65 & $4.57 y$ & 1.45 & $4.94^{2}$ & 1.45 & $4.86^{2}$ & 1.54 \\
\hline Product labelling & $4.17^{c, d}$ & 1.66 & $4.60^{c, d}$ & 1.48 & $4.95^{c}$ & 1.46 & $4.93^{c}$ & 1.55 \\
\hline Information campaigns & $4.08^{b, c}$ & 1.63 & $4.56^{\mathrm{b}, \mathrm{c}}$ & 1.43 & $4.93^{c}$ & 1.45 & $4.78^{c}$ & 1.53 \\
\hline Guide choice (medium) & $4.18^{y}$ & 1.70 & $4.58^{y}$ & 1.51 & $4.37 y$ & 1.65 & $4.47 \mathrm{y}$ & 1.71 \\
\hline Financial (dis)incentives & 4.34 & 1.72 & $4.74^{d}$ & 1.53 & $4.34^{b}$ & 1.73 & $4.47^{b}$ & 1.78 \\
\hline (dis)incentives & $4.02^{a, b}$ & 1.64 & $4.45^{a, b}$ & 1.47 & $4.42^{\mathrm{b}}$ & 1.57 & $4.47^{b}$ & 1.62 \\
\hline Restrict choice (high) & $3.90^{x}$ & 1.68 & $4.42^{x}$ & 1.54 & $4.19^{x}$ & 1.70 & $4.29^{x}$ & 1.76 \\
\hline Physical choice restriction & $3.96^{\mathrm{a}}$ & 1.70 & $4.51^{\mathrm{a}, \mathrm{b}, \mathrm{c}}$ & 1.56 & $4.06^{a}$ & 1.74 & $4.18^{\mathrm{a}}$ & 1.79 \\
\hline Advertising restriction & $3.85^{\mathrm{a}}$ & 1.64 & $4.34^{\mathrm{a}}$ & 1.52 & $4.34^{b}$ & 1.63 & $4.42^{b}$ & 1.69 \\
\hline \multicolumn{9}{|l|}{$\begin{array}{l}\text { Source and Source } x \\
\text { Intrusiveness }\end{array}$} \\
\hline Government & $4.02^{x}$ & 1.70 & $4.45^{x}$ & 1.55 & $4.29^{x}$ & 1.70 & $4.34^{x}$ & 1.76 \\
\hline Inforn & $4.10^{\mathrm{b}}$ & 1.64 & $4.57^{b, c}$ & 1.47 & $4.89^{d}$ & 1.47 & $4.81^{\mathrm{d}, \mathrm{e}}$ & 1.56 \\
\hline Guide & $4.11^{b}$ & 1.72 & $4.50^{\mathrm{b}}$ & 1.56 & $4.19^{b}$ & 1.70 & $4.28^{b}$ & 1.77 \\
\hline Restrict & $3.75^{a}$ & 1.69 & $4.25^{\mathrm{a}}$ & 1.59 & $3.88^{\mathrm{a}}$ & 1.74 & $3.98^{\mathrm{a}}$ & 1.82 \\
\hline Food manufacturers & $4.18^{y}$ & 1.66 & $4.64 y$ & 1.45 & $4.66^{y}$ & 1.56 & $4.71^{y}$ & 1.60 \\
\hline Inform choice & $4.15^{a}$ & 1.65 & $4.59^{b, c}$ & 1.44 & $4.98^{d}$ & 1.44 & $4.89^{e}$ & 1.53 \\
\hline Guide & $4.25^{c}$ & 1.66 & $4.69^{c}$ & 1.45 & $4.57^{c}$ & 1.58 & $4.65^{c, d}$ & 1.62 \\
\hline Restrict choice & $4.06^{b}$ & 1.64 & $4.59^{b, c}$ & 1.47 & $4.52^{c}$ & 1.58 & $4.62^{c}$ & 1.60 \\
\hline \multicolumn{9}{|l|}{$\begin{array}{l}\text { Approach/avoidance and } \\
\text { Approach/avoidance } x \\
\text { Intrusiveness } \\
\text { Encourage low-calorie }\end{array}$} \\
\hline choices & $4.33^{y}$ & 1.67 & $4.77^{y}$ & 1.44 & $4.85^{y}$ & 1.49 & $4.90^{y}$ & 1.55 \\
\hline Inform ch & $4.18^{c, d}$ & 1.64 & $4.61^{c}$ & 1.46 & $4.97^{d}$ & 1.43 & $4.91^{c}$ & 1.53 \\
\hline Guide choice & $4.41^{d}$ & 1.68 & $4.85^{d}$ & 1.42 & $4.79^{c}$ & 1.52 & $4.89^{c}$ & 1.55 \\
\hline $\begin{array}{l}\text { Restrict choice } \\
\text { Discourage high-calorie }\end{array}$ & - & - & - & - & - & - & - & - \\
\hline choices & $3.95^{x}$ & 1.67 & $4.41^{x}$ & 1.53 & $4.25^{x}$ & 1.68 & $4.30^{x}$ & 1.73 \\
\hline Infor & $4.07^{b, c}$ & 1.66 & $4.54^{b, c}$ & 1.46 & $4.25^{x}$ & 1.48 & $4.79^{c}$ & 1.55 \\
\hline Guide choice & $3.95^{a, b}$ & 1.67 & $4.34^{a}$ & 1.55 & 3.97 & 1.68 & $4.05^{a}$ & 1.75 \\
\hline Restrict choice & $3.90^{\mathrm{a}}$ & 1.67 & $4.42^{a, b}$ & 1.54 & $4.20^{\mathrm{b}}$ & 1.69 & $4.30^{b}$ & 1.74 \\
\hline
\end{tabular}

$x, y, z$ Superscripts for post-hoc comparisons for main effects. $a, b, c, c, d, e$ Superscripts for post-hoc comparisons for interaction effects. Dissimilar superscript letters within rows indicate significant differences, based on Games-Howell post hoc test $(p<0.05)$. Superscripts apply to a single column and single attribute part. 
Table 4.6: Means of the effect sizes (partial $\eta^{2}>0.015$ ) for snack food interventions.

\begin{tabular}{|c|c|c|c|c|c|c|c|c|}
\hline \multirow{2}{*}{$\begin{array}{l}\text { Main- and Interaction } \\
\text { Effects }\end{array}$} & \multicolumn{2}{|c|}{$\begin{array}{c}\text { Personal } \\
\text { Effectiveness }\end{array}$} & \multicolumn{2}{|c|}{$\begin{array}{c}\text { Societal } \\
\text { Effectiveness } \\
\end{array}$} & \multicolumn{2}{|c|}{ Fairness } & \multicolumn{2}{|c|}{ Acceptance } \\
\hline & Mean & SD & Mean & SD & Mean & SD & Mean & SD \\
\hline \multicolumn{9}{|l|}{$\begin{array}{l}\text { Intrusiveness and } \\
\text { Intrusiveness x Type }\end{array}$} \\
\hline Inform choice (low) & $4.13^{y}$ & 1.66 & $4.44^{x}$ & 1.49 & $4.98^{z}$ & 1.51 & $4.93^{z}$ & 1.57 \\
\hline Product labelling & $4.16^{b}$ & 1.72 & $4.38^{a, b}$ & 1.55 & $4.99^{d}$ & 1.55 & $4.95^{c}$ & 1.62 \\
\hline Information campaigns & $4.10^{b}$ & 1.61 & $4.50^{\mathrm{b}}$ & 1.43 & $4.96^{d}$ & 1.49 & $4.91^{\mathrm{c}}$ & 1.52 \\
\hline Guide choice (medium) & $4.19^{y}$ & 1.71 & $4.55^{y}$ & 1.50 & $4.42^{y}$ & 1.72 & $4.51^{y}$ & 1.75 \\
\hline Financial (dis)incentives & $4.27^{c}$ & 1.75 & $4.61^{b}$ & 1.54 & $4.34^{\mathrm{b}}$ & 1.80 & $4.47^{b}$ & 1.83 \\
\hline Assortment (dis)incentives & $4.11^{b}$ & 1.66 & $4.49^{b}$ & 1.46 & $4.51^{\mathrm{c}}$ & 1.64 & $4.54^{\mathrm{b}}$ & 1.66 \\
\hline$\underline{\text { Restric }}$ & $3.96^{x}$ & 1.69 & $4.35^{x}$ & 1.56 & $4.27^{x}$ & 1.72 & $4.33^{x}$ & 1.78 \\
\hline ce restriction & $4.00^{a, b}$ & 1.72 & $4.38^{\mathrm{a}, \mathrm{b}}$ & 1.61 & $4.00^{\mathrm{a}}$ & 1.78 & $4.07^{a}$ & 1.84 \\
\hline Advertising restriction & $3.81^{a}$ & 1.66 & $4.22^{\mathrm{a}}$ & 1.50 & $4.53^{c}$ & 1.64 & $4.59^{b}$ & 1.67 \\
\hline \multicolumn{9}{|l|}{$\begin{array}{l}\text { Source and Source } x \\
\text { Intrusiveness }\end{array}$} \\
\hline Government & $4.03^{x}$ & 1.72 & $4.38^{x}$ & 1.54 & $4.31^{x}$ & 1.76 & $4.35^{x}$ & 1.79 \\
\hline Inforn & $4.11^{\mathrm{b}}$ & 1.68 & $4.44^{b}$ & 1.49 & $4.94^{d}$ & 1.53 & $4.89^{d}$ & 1.61 \\
\hline Guide choice & $4.09^{b}$ & 1.73 & $4.45^{b}$ & 1.54 & $4.20^{\mathrm{b}}$ & 1.78 & $4.28^{\mathrm{b}}$ & 1.81 \\
\hline Restr & $3.81^{a}$ & 1.72 & $4.18^{a}$ & 1.61 & $3.89^{a}$ & 1.75 & $3.97^{\mathrm{a}}$ & 1.82 \\
\hline Food mar & $4.21^{y}$ & 1.67 & $4.56 y$ & 1.48 & $4.73^{y}$ & 1.60 & $4.78^{y}$ & 1.63 \\
\hline Inform choice & $4.15^{b, c}$ & 1.65 & $4.43^{b}$ & 1.49 & $5.01^{d}$ & 1.49 & $4.97^{d}$ & 1.53 \\
\hline Guide choice & $4.29^{c}$ & 1.68 & $4.65^{c}$ & 1.46 & $4.64^{c}$ & 1.63 & $4.73^{c, d}$ & 1.53 \\
\hline Restrict choice & $4.10^{\mathrm{b}}$ & 1.66 & $4.52^{b, c}$ & 1.49 & $4.64^{c}$ & 1.62 & $4.69^{c}$ & 1.66 \\
\hline \multicolumn{9}{|l|}{$\begin{array}{l}\text { Approach/avoidance and } \\
\text { Approach/avoidance } \mathbf{x} \\
\text { Intrusiveness } \\
\text { Encourage low-calorie }\end{array}$} \\
\hline choices & $4.37 y$ & 1.67 & $4.72^{y}$ & 1.43 & $4.92^{y}$ & 1.54 & $4.98^{y}$ & 1.55 \\
\hline$\overline{\text { Inform }}$ choice & $4.18^{c}$ & 1.64 & $4.49^{b}$ & 1.46 & $5.01^{c}$ & 1.49 & $4.98^{c}$ & 1.55 \\
\hline Guide choice & $4.47^{d}$ & 1.68 & $4.83^{c}$ & 1.40 & $4.88^{c}$ & 1.56 & $4.98^{c}$ & 1.56 \\
\hline $\begin{array}{l}\text { Restrict choice } \\
\text { Discourage high-cal }\end{array}$ & - & - & - & - & - & - & - & - \\
\hline choices & $3.96^{x}$ & 1.69 & $4.32^{x}$ & 1.54 & $4.28^{x}$ & 1.73 & $4.32^{x}$ & 1.78 \\
\hline 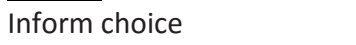 & $4.08^{b, c}$ & 1.69 & $4.38^{a, b}$ & 1.52 & $4.95^{c}$ & 1.53 & $4.88^{c}$ & 1.59 \\
\hline Guide choice & $3.91^{\mathrm{a}}$ & 1.69 & $4.27^{a}$ & 1.54 & $3.96^{a}$ & 1.74 & $4.03^{a}$ & 1.80 \\
\hline Restrict choice & $3.96^{a, b}$ & 1.70 & $4.35^{a}$ & 1.56 & $4.27^{b}$ & 1.72 & $4.33^{\mathrm{b}}$ & 1.78 \\
\hline
\end{tabular}

$x, y, z$ Superscripts for post-hoc comparisons for main effects. ${ }^{a, b, c, d, d, e}$ Superscripts for post-hoc comparisons for interaction effects. Dissimilar superscript letters within rows indicate significant differences, based on Games-Howell post hoc test $(p<0.05)$. Superscripts apply to a single column and single attribute part.

Table 4.7 shows the results from the analysis of variance with and without adding the intervention-specific beliefs to the model, to explore whether they mediate the effects of intrusiveness and intervention attributes on acceptance of snack choice 
interventions. Compared to the effect sizes without adding intervention-specific beliefs, the effects of intrusiveness and intervention attributes on acceptance almost disappeared. Subsequently, we performed Sobel tests (Sobel, 1982) to test the mediating effect of each intervention-specific belief on the relation between each level of intrusiveness and acceptance. All Sobel tests yielded significant mediation effects $(p<0.001)$. In support of Hypothesis 3 , we conclude that the effect of intrusiveness on acceptance of interventions is mediated by the perceived personaland societal effectiveness, and the perceived fairness of interventions.

Table 4.7. Effect sizes (partial $\eta^{2}$ ) of intrusiveness and intervention attributes on acceptance of interventions, with (left column) and without (right column) adding intervention-specific beliefs to the model (snack foods and beverages combined).

\begin{tabular}{lll}
\hline Main- and Interaction Effects & Partial $\eta^{2}$ & Partial $\eta^{2}$ \\
\hline Intervention-specific beliefs & & \\
Personal effectiveness & $0.023^{* * *}$ & \\
Societal effectiveness & $0.035^{* * *}$ & \\
Fairness & $0.447^{* * *}$ & \\
Intrusiveness & $0.001^{* * *}$ & $0.016^{* * *}$ \\
Source & $0.003^{* * *}$ & $0.027^{* * *}$ \\
Location & 0.000 & 0.000 \\
Approach/avoidance & $0.005^{* * *}$ & $0.050^{* * *}$ \\
Type & $0.001^{* *}$ & $0.001^{* * *}$ \\
Severity & 0.000 & 0.000 \\
Intrusiveness $x$ attributes ${ }^{1}$ & 0.007 & $0.032^{* * *}$ \\
Total $R^{2}$ & $0.717(0.716)$ & $0.127(0.126)$ \\
\hline * $p<0.05, * * p<0.01, * * * p<0.001$. & \\
1The sum of interactions of intrusiveness with source, location, approach/avoidance, and \\
severity.
\end{tabular}

\subsection{Discussion}

The present study investigated the processes underlying acceptance of intervention strategies for low-calorie snack choices and examined differences in acceptance of interventions with varying levels of intrusiveness. A total of 128 systematically varied intervention strategies were evaluated with respect to three intervention-specific beliefs (perceived personal- and societal effectiveness and the perceived fairness) and overall acceptability.

We found that perceptions of personal- and societal effectiveness, and fairness positively influence acceptance of interventions for low-calorie snack choices. This is 
in line with the findings from the transport policy domain (Eriksson et al., 2008) and also confirms the findings from a qualitative study on acceptance of interventions in the food-choice domain (Bos et al., 2013). Our results are robust across the application areas of beverages and snack foods, thereby implying that the relation between intervention-specific beliefs and acceptance is generalizable across foodrelated product categories.

Perceived fairness emerged as the strongest predictor of intervention acceptance. While being related to perceived fairness, the perceived personal- and societal effectiveness both had unique effects on acceptance as well. This indicates that despite their considerable overlap, both types of intervention-specific beliefs should be considered when maximizing the acceptance of food choice interventions.

In line with literature on changing health-related behaviours (Pechey et al., 2014), we show a greater acceptance of less intrusive interventions (e.g., product labelling). However, the present study extends this literature by showing that the effect of intrusiveness on acceptance is mediated by perceived personal- and societal effectiveness, and perceived fairness of interventions. Herewith, we provide insight into processes underlying acceptance and furthermore show that low levels of perceived effectiveness and perceived fairness form barriers for acceptance. Particularly for intrusive interventions, this finding provides novel starting points for increasing acceptance.

There seems to be a discrepancy between perceptions of effectiveness and actual effectiveness of interventions. While research shows that more intrusive interventions are more effective (Brambila-Macias et al., 2011; Horgen \& Brownell, 2002), we found that perceptions of personal- and societal effectiveness of providing information (low intrusiveness) and guiding choice (medium intrusiveness) were higher than for restricting choice (high intrusiveness). This could be due to reactance, which occurs when people are feeling restricted in their freedom of choice (Brehm, 1966).

The variations in operationalization at the different levels of intrusiveness mostly had very limited, if any, effect on the evaluations of interventions. It was seen as irrelevant on which of the two locations the intervention was implemented (supermarkets versus points-of-purchase on gas- and train stations), with which of the two intervention types within the level of intrusiveness this was executed (product labelling versus information campaigns; financial (dis)incentives versus 
assortment (dis)incentives; physical choice restriction versus advertising restriction), and how strong it attempted to do so. We did find that people perceived interventions that discourage high-calorie choices to be less effective than strategies that encourage low-calorie choices. Literature, however, suggests that interventions that discourage bad choices are, in fact, more effective (Epstein et al., 2010; Giesen et al., 2012). Some scholars therefore propose that knowledge about intervention effectiveness should be disseminated clearly to the public in order to increase acceptance (Pechey et al., 2014; Promberger et al., 2012).

Interestingly, our study also revealed that perceptions of fairness and acceptance were lower for interventions implemented by the government, as compared to food manufacturers. This could be explained by people perceiving intrusive government interventions to be paternalistic (Bos et al., 2013), while similar interventions by food manufacturers are probably seen as a natural mechanism of the free market. Low acceptance of government interventions could pose problems for implementation, which would be a delicate matter since experts in the area of food policies stress that governments have a significant role to play in stimulating healthy eating behaviours (Nestle \& Jacobson, 2000; Waterlander, Steenhuis, De Vet, Schuit, \& Seidell, 2010). More research on ways to increase acceptance of intrusive government interventions thus is required.

Contrary to the expectations, we did not find the hypothesized moderating effect of the barrier profile of the "receiver of the intervention" on the relation between intrusiveness and perceived effectiveness. We did find, however, that people with the no-barrier and lack-of-opportunity profiles perceived interventions of all three intrusiveness levels to be more effective, more fair and, therewith, more acceptable than those with the lack-of-motivation profile. Since both the no-barrier and the lack-of-opportunity profile are characterized by high levels of motivation to choose low-calorie snacks, this suggest that the strength of the motivation to behave has an effect on perceptions of effectiveness and fairness. This is in line with what has been found in an earlier study by Eriksson et al. (2008).

Where food policy research suggests that the perceived personal responsibility moderates the effect of intrusiveness on perceived fairness and acceptance of interventions (Lusk \& Ellison, 2013; Pearl \& Lebowitz, 2014), the present study only found a negligibly small interaction effect. The results did show a larger, but still small direct effect, which indicates that people who attribute responsibility for food choice to factors outside personal control perceive interventions that guide and 
restrict choice to be more fair and acceptable, as compared to people who see food choice mainly as a personal responsibility. This direct effect of perceived responsibility on acceptance of more intrusive interventions has been implied in literature as well (Hilbert et al., 2007; Levitsky \& Pacanowski, 2012).

The present study comes with a few limitations. As the type of data does not allow one to establish causality, it is possible that causality between intervention acceptance and perceptions of effectiveness and fairness runs in the opposite direction of what is suggested by our model in Figure 6, or could even be bidirectional. It could for instance be that when people initially have a positive feeling towards an intervention, they are more likely to perceive this intervention to be more effective as well. While the proposed model is grounded in earlier research on acceptance of interventions, experimental studies are needed to rule out different relations between the intervention evaluations.

Even though we used the Nuffield intervention ladder (2007), which specifically ranks interventions according to their intrusiveness, it is possible that respondents' perceptions of intrusiveness were different. In particular 'restricting advertising' was evaluated as a relatively acceptable intervention, which could indicate that people perceive this as a less intrusive intervention that guides rather than restricts choice. In general, however, our results show that interventions that are classified as more intrusive in the Nuffield ladder were accepted less. As this is in line with earlier research that shows when people perceive interventions to be intrusive, they are less likely to accept them (Laurin et al., 2012), this suggests that the objective and subjective intrusiveness likely are quite similar. Since, to our knowledge, no research has been done on perceptions of intervention intrusiveness, future research should encompass the assessment of perceptions of intervention intrusiveness as well.

As the present study was primarily concerned with examining if and how perceptions of effectiveness and fairness predicted acceptance across a wide range of intervention strategies, we assessed these constructs with single items. Although it is common to measure intervention-specific beliefs this way (Eriksson et al., 2008; Promberger et al., 2011), it is possible that people differ in how they construct perceptions of effectiveness and fairness of interventions (Bos et al., 2013). Future research should therefore look into different connotations of intervention-specific beliefs and examine their unique effects on acceptance. 
The present study elicited evaluations of hypothetical strategies to stimulate lowcalorie snack choices. Research suggest that the stage of implementation of an intervention (i.e., the certainty that an intervention will be implemented) influences evaluations drastically (Diepeveen et al., 2013; Laurin et al., 2012). Therefore it would be valuable to see if and how snack choice interventions in various stages of implementation (e.g., implementation $20 \%$ certain versus $80 \%$ certain) are evaluated differently than the hypothetical strategies in the present study.

\subsection{Conclusions}

Perceptions of personal- and societal effectiveness, and fairness, influence acceptance of interventions that stimulate healthy food choices. Particularly when interventions are intrusive, it is important for policy makers to address these intervention-specific beliefs to maximize intervention acceptance. We encourage future research on food choice interventions to build upon the present study's results, thereby further unravelling the processes underlying intervention acceptance. 

5

\section{PROMOTING HEALTHY CHOICES FROM VENDING MACHINES}

Effectiveness and consumer evaluations of four types of interventions

This chapter is submitted as:

Bos, C., Van der Lans, I., Van Kleef, E., Van Trijp, H.C.M. (2016) Promoting healthy choices from vending machines: effectiveness and consumer evaluations of four types of interventions 


\section{Abstract}

\section{Background}

Vending machines provide, often relatively energy-dense, snack foods and beverages in a wide variety of points-of-purchase. Vending-machine interventions that stimulate low-calorie choices therefore may substantially decrease calorie intake. The aim of the present study is to examine the effects of intervention intrusiveness on the effectiveness and acceptance of vending-machine interventions, and evaluations of the choices made, as prerequisites for long-term intervention effectiveness.

\section{Methods}

In a between-subjects design experiment $(\mathrm{N}=206)$, respondents were asked to purchase a snack and a beverage from a vending machine. In addition to a nointervention condition, four types of interventions that were derived from the Nuffield intervention ladder were implemented. A post-choice questionnaire included items concerning intervention acceptance, assortment and choice evaluations, and barriers for choosing low-calorie snacks.

\section{Results}

Compared to the no-intervention condition, restricting choice led to more lowcalorie choices (39\% vs. $78 \%$ ), while provision of calorie information, assortment incentives, and price disincentives did not lead to different choice behaviour. Furthermore, acceptance and choice evaluations were equally high across these intervention types.

\section{Conclusions}

Overall, the results suggest that restricting high-calorie options is a promising route to stimulate healthier choices from vending machines. As such, the present study provides intervention opportunities for governments and their potential allies in the combat against obesity, such as food manufacturers and the food service industry. 


\subsection{Introduction}

In an environment where tasty, convenient, and inexpensive high-calorie foods are present in abundance (Ni Mhurchu et al., 2013), consumers in the Western world increasingly struggle to adequately manage their caloric intake (Cohen et al., 2010). As a result, currently more than 1.5 billion people are overweight or obese (Swinburn et al., 2011). Intervention strategies that stimulate low-calorie food choice behaviour are therefore seen as indispensable tools to curb the current obesity epidemic: scholars stress that widespread governmental measures should be implemented to enforce lasting improvements in the population's dietary behaviours (Nestle \& Jacobson, 2000; Waterlander et al., 2010).

An increasingly large proportion of people's daily caloric intake originates from snacking (Popkin \& Duffey, 2010), which is the consumption of foods and beverages outside breakfast, lunch, and dinner. Important points-of-purchase for a variety of snack foods and beverages are vending machines; these provide snacking opportunities at numerous locations such as work- and school canteens, sport canteens, and train stations (Kocken et al., 2012). Vending machine density is particularly high at high schools and universities: around $75 \%$ to $80 \%$ of these institutions facilitate snack foods and beverages through one or more vending machines (Larson \& Story, 2010; Mikolajczak, Van den Berg, \& Bemelmans, 2012). Since vending machine use is associated with detrimental dietary behaviours (Wiecha, Finkelstein, Troped, Fragala, \& Peterson, 2006), implementing interventions that stimulate low-calorie choices in vending machines is a promising avenue to lower caloric intake through snacking.

Literature on intervention strategies for healthy food choices brings forward three concepts as important conditions for long-term intervention effectiveness. First, there needs to be evidence of short-term effectiveness; if there is no indication that interventions lead to the desired behaviour on the short term, long-term effectiveness is unlikely (Traill et al., 2010). Second, interventions need to be accepted by consumers and food manufacturers (Kelly et al., 2009), because a lack of intervention acceptance potentially leads to discontinuation or even nonimplementation of (proposed) policies (e.g. the Danish sugar tax) (Jou \& Techakehakij, 2012). Third, interventions should not lead to frustration and dissatisfaction when making actual food choices, as these negative choice evaluations potentially lead consumers to search for alternative choice situations 
(Proudfoot \& Kay, 2014). The following section provides an overview of these three lines on research.

Knowledge on the effectiveness of vending-machine interventions is limited, but the available research suggests that greater availability and lower prices of healthier options can be effective in stimulating healthy choices (S. A. French et al., 2010; S. A. French et al., 2001; Grech \& Allman-Farinelli, 2015). For the effectiveness of healthy eating interventions in general, one can conclude that many of the interventions that have been implemented during the past decades mostly aimed to support informed choice, for instance through food labelling and information campaigns (Capacci et al., 2012). These interventions, however, have largely failed to bring about substantive behaviour change (Brambila-Macias et al., 2011). In contrast, more intrusive interventions such as taxing and restricting unhealthy foods, show more promise in being effective in changing behaviour (Nederkoorn et al., 2011; Van Kleef et al., 2012). In line with this finding, the need for more intrusive governmental interventions to combat the obesity problem has been expressed by many public health experts (Gortmaker et al., 2011; Swinburn et al., 2011).

Whether interventions will lead to behaviour change furthermore depends on individual characteristics, specifically on which barriers people have for choosing healthily (Rothschild, 1999). In his Motivation-Opportunity-Ability (MOA) framework, Rothschild argues that there are three main barriers for desired behaviour (motivation, opportunity, and ability), and that there needs to be a 'fit' between barrier and intervention intrusiveness to achieve maximum intervention effectiveness. Concretely, if people lack ability, interventions should focus on increasing knowledge about (un)healthiness of choices; if they lack opportunity, interventions should focus on increasing availability and accessibility of healthy choices; and if motivation is lacking, interventions should focus on a combination of these interventions. If these measures are not effective, interventions should be even more intrusive in order to coerce people to choose healthily, for instance by restricting and even eliminating unhealthy options.

A pressing issue with highly intrusive food choice interventions is that they are often met with resistance, both by food manufacturers and consumers (Bos et al., 2013; Diepeveen et al., 2013). In their comprehensive review on acceptance of government interventions to change health-related behaviours, Diepeveen et al. conclude that acceptance is strongly influenced by interventions' level of intrusiveness. 
Interventions that are less intrusive, such as information provision, were accepted more than those that are more intrusive, such as restriction of unhealthy choices.

Research on the cognitive process underlying acceptance of food choice interventions indicates that the relation between intervention intrusiveness and acceptance is mediated by two intervention-specific beliefs: the perceived effectiveness and the perceived fairness (Bos, Van der Lans, Van Rijnsoever, \& Van Trijp, 2015a). The perceived effectiveness refers to whether people think interventions will be successful in stimulating healthy food choices, thereby differentiating between effectiveness for themselves and for society as a whole. The perceived fairness, on the other hand, refers to the extent to which people perceive interventions to be a fair way of stimulating healthy food choices. Bos et al. (2015a) conclude that across different levels of intrusiveness perceptions of personal and societal effectiveness only differ marginally. When looking at the perceived fairness, however, the more intrusive interventions become, the less they are perceived as fair. They furthermore conclude that acceptance of interventions is influenced by barriers for healthy choices: people who are less motivated to choose healthily accept all types of interventions less than those who are more motivated.

In addition to its effect on intervention effectiveness and intervention acceptance, higher levels of intervention intrusiveness can also evoke negative affective responses towards choice situations (Laurin et al., 2012). Applied to a food choice context, these choice evaluations manifest themselves as frustration during the process of making choices as well as (dis)satisfaction with the choices made. Frustration and dissatisfaction are generally higher for more intrusive interventions, as these restrict freedom of choice more. Whether frustration and dissatisfaction occur, however, likely depends on how people evaluate a post-intervention assortment, as positive perceptions of assortment attractiveness are known to lead to higher choice satisfaction (Diehl \& Poynor, 2010).

Summarizing, interventions and their intrusiveness influence people on a behavioural, cognitive, and affective level. The present study aims to contribute to the existing literature in two ways. First, food choice experiments generally focus on the effectiveness of a single intervention type or they compare the effectiveness of two intervention types, most often provision of information versus price (dis)incentives (Giesen et al., 2011; Horgen \& Brownell, 2002). The present study implements four intervention types from the Nuffield intervention ladder (2007) that differ regarding the level of intrusiveness; this enables gaining a better 
understanding of the effect of intrusiveness on intervention effectiveness. Second, intervention evaluations often have been researched in terms of hypothetical policies (Bos et al., 2015a), in which direct consequences of the interventions are not experienced. Other papers mostly assess evaluations of existing policies using surveys (Diepeveen et al., 2013), in which outside influences cannot be controlled. To complement both types of research, the present study examines acceptance of interventions when they are both incentive aligned and implemented in a controlled, realistic setting.

The present study simultaneously investigates the effectiveness and acceptance of real-life vending-machine interventions and also assesses evaluations of the choices that are made. To examine differences between various levels of intrusiveness, four intervention types are implemented (from low- to high intrusiveness): providing calorie information, guiding choice through assortment incentives, guiding choice through price disincentives, and restricting choice. Subsequently, we examine whether interventions are evaluated differently if they are experienced in a realistic setting, compared to when they are presented as hypothetical interventions. Figure 5.1 provides a conceptual overview of the present study.

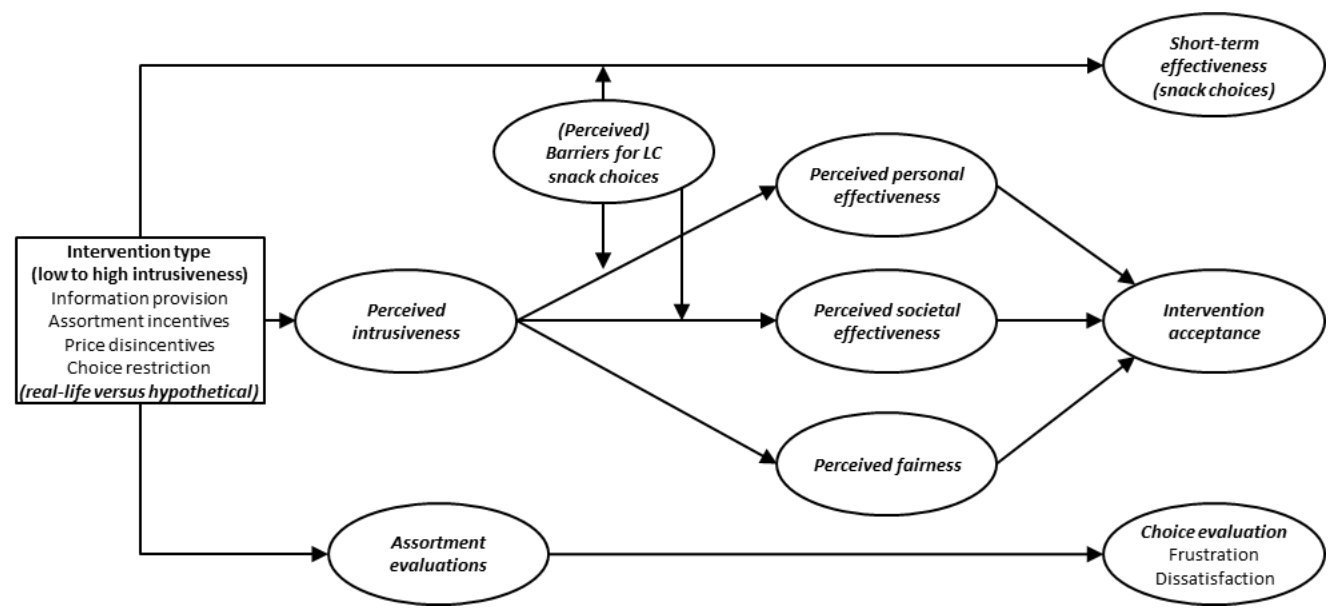

Figure 5.1: Overview of the present study's conceptual framework 


\subsection{Methodology}

\section{Experimental design of study}

The study employed a between subjects design in which intervention type was manipulated. Respondents were assigned to one of five conditions. In addition to a control condition there were four intervention conditions that each involved a different type of intervention strategy. These interventions differed regarding the level of intrusiveness, which was derived from Nuffield's intervention ladder (2007). Table 5.1 gives an overview of the characteristics of the experimental conditions. In the condition with the lowest intrusiveness, the information condition, green and red labels were added to the pricing information on the facings of the vending machine. These labels were not present in the other intervention conditions. In the availability condition, the composition of the assortment was changed to 12 facings for low-calorie and 6 facings for high-calorie products, without affecting the freedom of choice (all products remained but with a different number of facings). In the price condition, prices of high-calorie products were increased by $33 \%$, while prices for low-calorie products remained the same. In the condition with the highest intrusiveness, the restriction condition, half of the most high-calorie products were eliminated from the machine and the facings were filled with already present lowcalorie products.

Table 5.1: Overview of the characteristics of the five experimental conditions

\begin{tabular}{|c|c|c|c|c|c|c|}
\hline & \multirow[t]{2}{*}{ Labels } & \multicolumn{2}{|c|}{$\begin{array}{c}\text { Facings } \\
\text { snack foods } \\
\text { (\# products) }\end{array}$} & \multicolumn{2}{|c|}{$\begin{array}{c}\text { Facings } \\
\text { beverages }\end{array}$} & \multirow{2}{*}{$\begin{array}{l}\text { Prices }^{1} \\
\mathrm{HC} \\
\end{array}$} \\
\hline & & LC & $\mathrm{HC}$ & LC & $\mathrm{HC}$ & \\
\hline \multicolumn{7}{|l|}{ Condition } \\
\hline Control & No & $6(6)$ & $12(6)$ & 2 & 2 & $€ 0.75$ \\
\hline Information & Yes & $6(6)$ & $12(6)$ & 2 & 2 & $€ 0.75$ \\
\hline Availability & No & $12(6)$ & $6(6)$ & 3 & 1 & $€ 0.75$ \\
\hline Price & No & $6(6)$ & $12(6)$ & 2 & 2 & $€ 1.00(+33 \%)$ \\
\hline Restriction & No & $12(6)$ & $6(3)$ & 3 & 1 & $€ 0.75$ \\
\hline
\end{tabular}

1Prices of low-calorie snacks were fixed at $€ 0.75$ across all conditions

\section{Assortment in vending machine}

Stimuli were presented in a vending machine containing 18 facings for snack foods and 4 facings for beverages, which varied depending on the intervention type. The snack foods and beverages were identified as frequently consumed snacks in earlier 
research (Bos et al., 2013). The 18 facings for snack foods were filled with an assortment consisting of 12 snack foods involving 3 types of snack foods appearing both as low- (i.e. <100 kcal) or high-calorie (i.e. >130 kcal) variants (Table 5.2). Additionally, an assortment of five beverages was used to fill the four facings for beverages. This division of products into low- and high-calorie categories is similar to caloric content classifications in other research (Kocken et al., 2015).

Table 5.2: Overview of low-calorie and high-calorie snack foods and beverages in the vending machine

\begin{tabular}{lll}
\hline Type & Low-calorie (<100 kcal) & High-calorie (>130 kcal) \\
\hline Candy bar & Milky Way & Mars $^{1}$ \\
& Kellogg's Chewy Delight & Kinder Bueno $^{1}$ \\
Cookie & Bolletje Goed Bezig Cranberry & Snelle Jelle Gingerbread \\
& Kellogg's Biscuit Moments & Bolletje Goed Bezig Naturel \\
Fruit/vegetable snack & Made Good Banana & Evergreen Forest Fruits \\
& Made Good Strawberry & Liga Haverkick Apple \\
Beverages & Lipton Ice Tea Green & Coca Cola Regular \\
& Coca Cola Green & Fanta Regular \\
& Fanta Light & \\
\hline
\end{tabular}

${ }^{1}$ Not included in assortment of restriction condition

The assortment of the control condition was based on earlier research on vending machines, which states that in a realistic setting a vending machine contains more high-calorie facings than low-calorie facings (Scholtens, Middelbeek, Rutz, Buijs, \& Bemelmans, 2010). Additionally, all the products were priced $€ 0.75$, which is similar to the selling prices of other vending machines on the university campus. The interventions used in the experimental conditions were chosen based on earlier research by Bos et al. (2015a), where they were identified as less accepted interventions within their intervention type.

\section{Procedure}

Data was collected in September of 2015 in the research room of the Social Sciences building of the Wageningen University. Data collection was divided into two stages: one stage concerned the intervention implemented in the vending machine, the subsequent stage concerned the remaining interventions that were not experienced by the respondent and thus were presented on paper. 


\section{Vending machine intervention (Stage 1)}

Respondents first performed an unrelated task for 5-10 minutes and subsequently received a reward amounting 5 euros with which they were instructed to buy a snack food and a beverage from a vending machine. Respondents then bought these snacks in one of the five conditions (control, information, availability, price, and restriction). The money that was left after purchases from the vending machine remained in respondents' possession. The environment in which the snack choices were made was kept constant: there was no time pressure, there were no other people present who could potentially influence choice, and lighting and temperature were kept constant as well.

After choosing one snack food and one beverage from the vending machine, respondents were instructed to fill out a questionnaire. First, they were asked to explain why they chose the products and to rate the assortment of the vending machine. In the second part, respondents in the four intervention conditions were told that an intervention was implemented in the vending machine. They were then asked if they had noticed the intervention (yes/no). Subsequently respondents were informed about the rationale behind the intervention. Because the stage of implementation of an intervention influences intervention evaluations (Diepeveen et al., 2013; Laurin et al., 2012), and to maximize personal relevance, respondents were informed that the intervention was very likely to come into effect: "We implemented this intervention in the context of a government project, which aims to find the most suitable ways to stimulate low-calorie snack choices. It is very likely that new policies regarding stimulating healthier food choice behaviour will include this intervention". Following this, respondents evaluated this intervention on effectiveness, fairness, and acceptability.

\section{Remaining (hypothetical) interventions (Stage 2)}

In the second stage, respondents were shown the alternative interventions on an A4 paper. These were graphical depictions of the three interventions that were implemented in the other experimental conditions (hence not experienced by the respondent). Again, they rated these on effectiveness, fairness, and acceptability. To investigate whether perceptions of intrusiveness aligned with the Nuffield intervention ladder classification of intrusiveness, respondents were then asked to rate all four interventions on their perceived intrusiveness. In the final part of the questionnaire respondents answered questions regarding individual characteristics. These consisted of the motivation and ability to choose low-calorie over high-calorie snacks in everyday life and demographic variables. 
After completing the questionnaire, respondents who were assigned to an intervention condition were debriefed regarding the rationale of the intervention. They were told that "even though the government is looking into ways to stimulate healthier food choice behaviour, at this point they have not yet decided how this will be shaped; it is therefore not certain that this intervention will come into effect".

\section{Respondents}

Recruitment of respondents occurred through handing out flyers and sending emails to students. Potential respondents were told that they would receive $€ 3.50$ and a snack and beverage of choice for participating in two short surveys, which would take 15-20 minutes in total. 206 respondents participated, of which $77 \%$ were women. Mean age was 20.67 (SD = 3.29).

\section{Measures}

\section{Short term effectiveness (snack choices)}

Interventions were regarded as effective when respondents chose a low-calorie product.

\section{Reasons for choosing snacks}

Reason for choosing snack foods and beverages were elicited by asking the openended question "Can you shortly elaborate on why you chose these products?". This was assessed separately for snack foods and beverages.

\section{Intervention acceptance}

Acceptance of intervention strategies was measured on a 7-point Likert-scale, with labels ranging from 'completely disagree' to 'completely agree', and consisted of 1 item: "I support the implementation of this strategy". This item was adapted from Bos et al. (2015a).

Intervention-specific beliefs: perceived effectiveness and perceived fairness Perceptions of effectiveness and fairness of intervention strategies were rated on a 7-point Likert-scale, with labels ranging from 'completely disagree' to 'completely agree'. Perception of effectiveness were divided into personal- ("This strategy would lead me to choose low-calorie over high-calorie beverages/snack foods more often than I normally do") and societal effectiveness ("This strategy would lead a lot of people to choose low-calorie over high-calorie beverages/snack foods more often 
than they normally do). Perceived fairness was measured with 1 item ("I think this strategy is a fair way to encourage people to choose low-calorie over high-calorie beverages/snack foods"). These items were adapted from Eriksson et al. (2008) and Bos et al. (2015a).

\section{Perceived intrusiveness}

To capture individual differences in perceptions of intervention intrusiveness, and to investigate whether respondents' perceptions of intervention intrusiveness aligned with the Nuffield intervention ladder classification, they were asked to rate all four interventions on their intrusiveness using 1 item ("To what extent do you feel that this intervention limits your freedom of choice?") on a 5-point scale ranging from 'not at all' to 'completely'.

Barriers to choose low-calorie over high-calorie snacks and subsequent segmentation

In total 14 items were used for measuring the Motivation (9 items) and the Ability (5 items) to choose low-calorie over high-calorie snacks in everyday life. Motivation was split up into cognitive (4 items) and affective attitude (4 items), and personal norm (1 item), while Ability consisted of the perceived knowledge about caloric values of snacks ( 4 items) and the perceived skills to interpret caloric information on packaging (1 item). A detailed description of the construction of the questionnaire can be found in Bos et al. (2015b).

To segment respondents based on their motivation and ability to choose low-calorie over high-calorie snacks in everyday life, we used the no-barrier and the lack-ofmotivation barrier profiles identified by Bos et al. (2015b) as reference. We calculated the sum of the squared Euclidean distances to the profile means on the motivation and ability factors in Bos et al., and subsequently respondents were assigned to the profile to which means he or she was closest. 142 respondents were found to have a no-barrier profile and 63 were profiled as having a lack-of-motivation profile.

\section{Choice evaluations}

Respondents evaluated their choice by indicating to what extent they found it frustrating to choose products from the vending machine ("I found it frustrating to choose products from this vending machine") and whether they were satisfied with the products they chose ("I am satisfied with the products I chose"). Both items were 
scored on a 7-point Likert-scale with labels ranging from 'completely disagree' to 'completely agree'.

\section{Assortment evaluations}

Respondents rated the assortment of the vending machine on three items. These were 7-point Likert-scale items, with labels ranging from 'completely disagree' to 'completely agree', and consisted of perceptions of assortment attractiveness ("The assortment of this vending machine was attractive"), healthiness ("The assortment of this vending machine was healthy"), and suitability for schools and universities ("I would expect such a vending machine in a school or university").

As a manipulation check, in each condition the implemented intervention was rated on one additional condition-specific assortment evaluation. For information this was "The vending machine helped me to distinguish between low- and high-calorie products", for availability this was "I found the assortment in the vending machine to be diverse", for price this was "I found the prices of the products in the vending machine to be reasonable", and for restriction this was "I missed some products in the assortment of this vending machine". To compare these condition-specific assortment evaluations to a baseline assortment, they were also included in the control condition. These items were scored on a 7-point Likert-scale with labels ranging from 'completely disagree' to 'completely agree'.

\section{Data analysis}

First, a logistic regression was performed to examine the effect of intervention type and the moderating effect of barrier profile on low-calorie choices.

Reasons for choosing snack foods and beverages were analysed and coded using the framework approach, meaning that the coding was performed deductively and inductively. The deductive part consisted of coding the reasons in terms of themes identified in The Eating Motivation Scale (TEMS) (Renner, Sproesser, Strohbach, \& Schupp, 2012). The inductive part consisted of coding additional themes that were not covered by the deductive coding.

To investigate whether the effect of perceived intrusiveness on intervention acceptance is mediated by intervention-specific beliefs (perceived personal- and societal effectiveness, and perceived fairness), Hayes Process macro (Hayes, 2012) for SPSS (version 2.13) was used. The effect of intervention type on choice 
evaluations (frustration and (dis)satisfaction) and the mediating effect of assortment evaluations were analysed using the same macro.

Last, ANOVA's were performed to see whether intervention types were evaluated differently by respondents who experienced the intervention in the realistic setting (in Stage 1), compared to respondents who evaluated it as a hypothetical intervention (in Stage 2).

\subsection{Results}

\section{Sample characteristics across conditions}

Table 5.3 shows that there were no differences between conditions regarding respondents' age, $\mathrm{BMI}$, and gender.

Table 5.3: Respondent characteristics in each of the intervention conditions

\begin{tabular}{|c|c|c|c|c|c|c|c|c|c|c|c|c|}
\hline & \multicolumn{12}{|c|}{ Condition } \\
\hline & \multicolumn{2}{|c|}{ Control } & \multicolumn{2}{|c|}{ Information } & \multicolumn{2}{|c|}{ Availability } & \multicolumn{2}{|c|}{ Price } & \multicolumn{2}{|c|}{ Restriction } & \multicolumn{2}{|c|}{ Total } \\
\hline & Mean & SD & Mean & SD & Mean & SD & Mean & SD & Mean & SD & Mean & SD \\
\hline $\mathrm{Age}^{1}$ & 21.17 & 5.17 & 19.58 & 2.40 & 20.43 & 2.09 & 20.85 & 2.91 & 21.28 & 2.36 & 20.67 & 3.29 \\
\hline $\mathrm{BMI}^{2}$ & 21.59 & 2.47 & 21.48 & 2.02 & 21.57 & 2.17 & 21.62 & 2.51 & 21.70 & 2.18 & 21.60 & 2.26 \\
\hline Women ${ }^{3}$ & $87 \%$ & & $68 \%$ & & $78 \%$ & & $73 \%$ & & $78 \%$ & & $77 \%$ & \\
\hline
\end{tabular}

${ }^{1} \mathrm{~F}(4,201)=1.825, p=0.125$

${ }^{2} \mathrm{~F}(4,199)=0.252, p=0.996$

${ }^{3}$ Chi-square $5.026, p=0.285$

Influence of intervention type and barrier profile on snack food and beverage choices

Chi-square tests were performed to examine the main effects of intervention type and barrier profile on the percentage of low-calorie choices. There was an effect of intervention type on low-calorie snack food choices; low-calorie choices were more prevalent in the restriction condition (78\%) than in the control and information condition (39\% and 35\%). There was no effect of intervention type on low-calorie beverage choices. Percentages of low-calorie choices in the different conditions can be found in Table 5.4. Additionally, there was a main effect of barrier profile on both low-calorie snack food and low-calorie beverage choices: respondents with a nobarrier profile chose more low-calorie products ( $57 \%$ low-calorie snack foods and 
$83 \%$ low-calorie beverages) than those with a lack-of-motivation profile (35\% lowcalorie snack foods and $68 \%$ low-calorie beverages). 


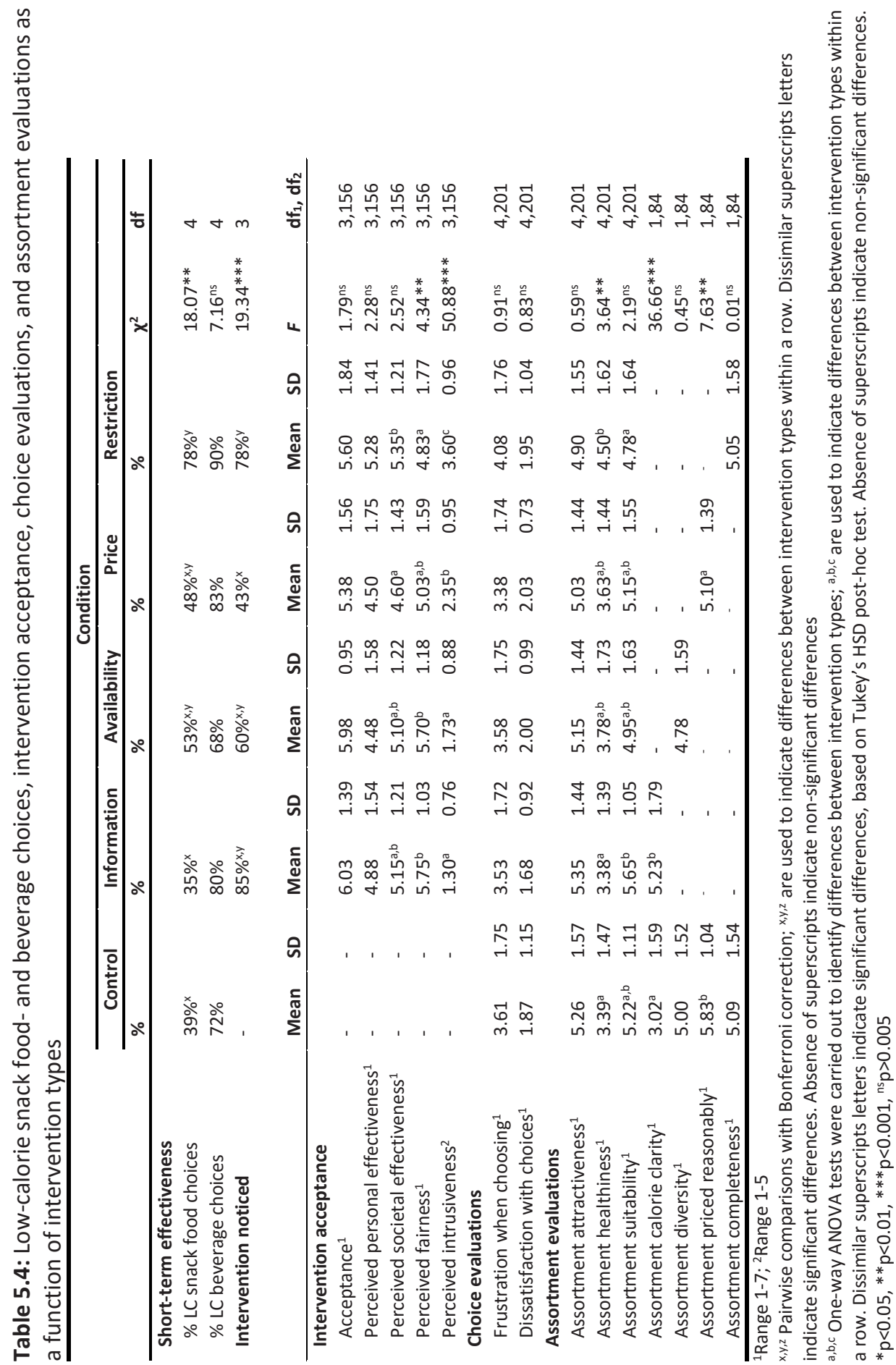


Although hypothesized in our model, for both snack food and beverage choices no interaction effect of intervention type and barrier profile on low-calorie choices was found. The results of the logistic regression can be found in Table 5.5.

Table 5.5: Logistic regression of intervention type and barrier profile on low-calorie snack food- and beverage choices

\begin{tabular}{|c|c|c|}
\hline & Snack foods & Beverages \\
\hline & $\mathrm{b}$ & $b$ \\
\hline \multicolumn{3}{|l|}{ Condition (Overall) } \\
\hline Condition(Information) & -0.117 & 0.401 \\
\hline Condition(Availability) & 0.538 & -0.306 \\
\hline Condition(Price) & 0.378 & 0.595 \\
\hline Condition(Restriction) & $1.755^{* * *}$ & 1.232 \\
\hline Barrier profile & $0.975^{* *}$ & $0.918^{*}$ \\
\hline Cox \& Snell R ${ }^{2}$ & 0.125 & 0.065 \\
\hline Nagelkerke $\mathbf{R}^{\mathbf{2}}$ & 0.166 & 0.101 \\
\hline
\end{tabular}

${ }^{*} p<0.05,{ }^{* *} p<0.01, * * * p<0.001$

Reasons for choosing products were similar across the snack food and beverage categories. The primary reasons for choosing snack foods were (in descending order) Liking (75), Health (34), Novelty (32), Need/hunger (23), and Price (11), while the primary reasons for choosing beverages were (in descending order) Liking (109), Health (17), Novelty (17), Need/hunger (14), and Habits (13). Chi-square tests with the Monte Carlo method (to control for low cell counts) showed that reasons for choosing products did not differ between intervention types, neither for snack foods (Chi-square 59.154, Monte Carlo $p=0.095)$ nor for beverages (Chi-square 59.132, Monte Carlo $p=0.083$ ). 
Influence of intervention type and barrier profile on acceptance and perceptions of effectiveness, fairness, and intrusiveness

To examine the main effects of intervention type and barrier profile on intervention evaluations, ANOVA's were performed. Acceptance did not differ between intervention types. There does seem to be a pattern in perceptions of personal-and societal effectiveness, with restriction being perceived as a more effective intervention, but these results were merely borderline significant. Perceptions of fairness were higher for the information and availability interventions, compared to the restriction intervention. A comparison of the means of acceptance, the perceived personal- and societal effectiveness, the perceived fairness, and the perceived intrusiveness of the interventions types can be found in Table 5.4.

Additionally, there was a main effect of barrier profile on intervention evaluations. Compared to respondents with a lack-of-motivation profile, respondents with the no-barrier profile perceived interventions to be more effective on a personal level ( 5.09 vs $4.08 ; F(1,157)=14.62, p<0.001)$, more effective on a societal level $(5.20$ vs $4.69 ; F(1,157)=5.37, p=0.022)$, and more fair $(5.50$ vs $4.90 ; F(1,157)=12.29, p=0.016)$. Consequently, they also accepted interventions more $(6.05$ vs $5.04 ; F(1,157)=17.18$, $p<0.001)$.

Respondents' perceptions of intervention intrusiveness generally aligned with the Nuffield intervention ladder classification of intrusiveness (Table 5.4). Except for the information and availability interventions, all interventions differed on perceived intrusiveness in the hypothesized direction. To account for differences in perceptions of intrusiveness between respondents, the analysis was continued with perceived intrusiveness as independent variable.

Prior to conducting the multiple linear regression analysis of intervention acceptance on perceived intrusiveness, correlations between acceptance, intervention-specific beliefs (perceived personal- and societal effectiveness, and perceived fairness) and perceived intrusiveness were inspected (Table 5.6). Acceptance, perceived personal and societal effectiveness, and perceived fairness were positively correlated. Acceptance and perceived fairness were negatively correlated with perceived intrusiveness, while there was no relation between perceptions of personal and societal effectiveness and perceived intrusiveness. 
Table 5.6: Correlations between acceptance, intervention-specific beliefs and perceived intrusiveness

\begin{tabular}{|c|c|c|c|c|c|}
\hline & Acceptance & $\begin{array}{c}\text { Personal } \\
\text { effectiveness }\end{array}$ & $\begin{array}{c}\text { Societal } \\
\text { effectiveness }\end{array}$ & Fairness & $\begin{array}{c}\text { Perceived } \\
\text { intrusiveness }\end{array}$ \\
\hline Acceptance & & $0.434 * * *$ & $0.409 * * *$ & $0.556 * * *$ & $-0.271^{* *}$ \\
\hline Personal effectiveness & & & $0.434 * * *$ & $0.178^{*}$ & 0.072 \\
\hline Societal effectiveness & & & & $0.304^{* *}$ & -0.016 \\
\hline Fairness & & & & & $-0.314 * * *$ \\
\hline $\begin{array}{l}\text { Perceived } \\
\text { intrusiveness }\end{array}$ & & & & & \\
\hline
\end{tabular}

${ }^{*} p<0.05,{ }^{* *} p<0.01,{ }^{* * *} p<0.001$

Figure 5.2 shows the effect of perceived intrusiveness on intervention acceptance, and the mediating effect of the perceived personal- and societal effectiveness, and the perceived fairness. Without adding the intervention-specific beliefs, perceived intrusiveness accounted for $7.4 \%(p=0.001)$ of the variance in intervention acceptance. When adding the intervention-specific beliefs, the direct effect of perceived intrusiveness accounted for $2.4 \%(p=0.009)$ of the variance in intervention acceptance. Since perceived fairness is the only intervention-specific belief to be related to perceived intrusiveness, the conclusion therefore is that the effect of perceived intrusiveness on intervention acceptance therefore is partially mediated by perceived fairness.

Although hypothesized in our model, no interaction effect of perceived intrusiveness and barrier profile on perceptions of personal- and societal effectiveness was found. 


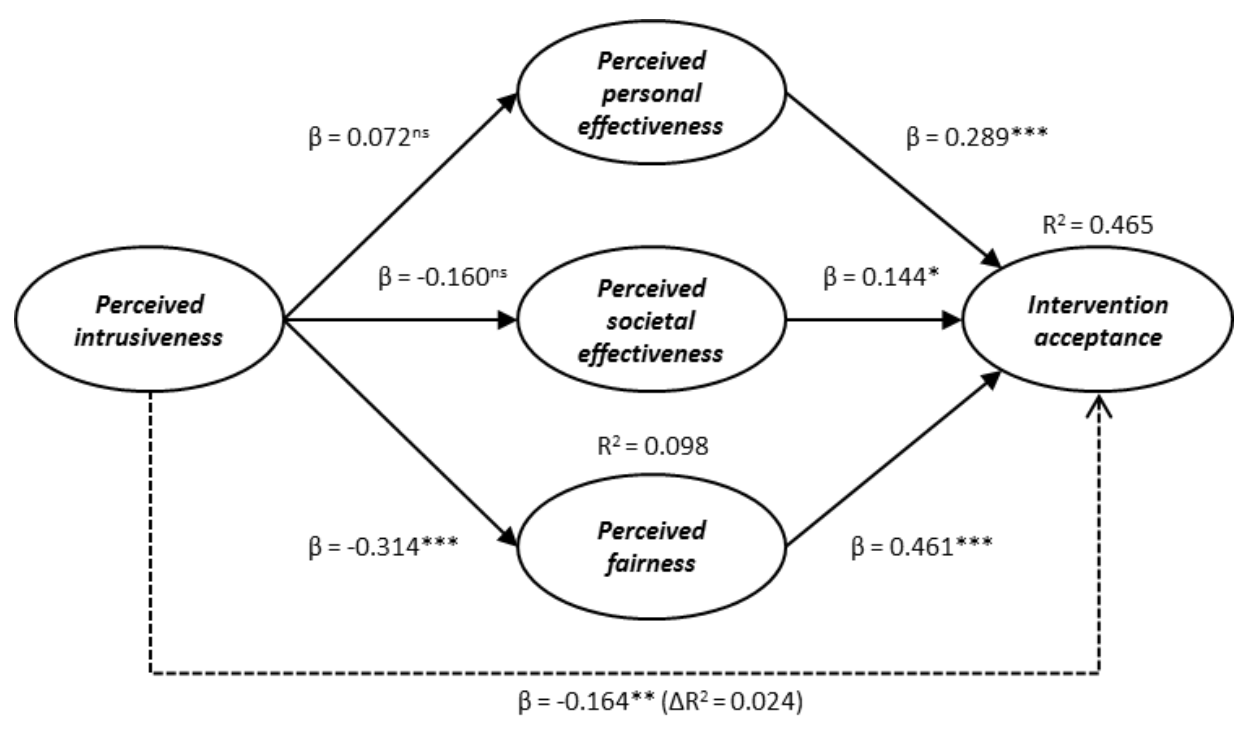

Figure 5.2: Mediation analysis

${ }^{*} p<0.05, * * p<0.01, * * * p<0.001$

Influence of intervention type on choice evaluations and assortment evaluations

To examine the effects of intervention type on choice evaluations and assortment evaluations, ANOVA's were performed. There were no main effects of intervention type on both types of choice evaluations: frustration when choosing a snack food and a beverage from the vending machine and the subsequent satisfaction with these choices did not differ (Table 5.4). The perceived attractiveness of the assortment also did not differ between intervention types. Perceptions of assortment healthiness were higher in the restriction condition than in the control and information condition. Assortment suitability for schools and universities only differed between the information and restriction condition, with the assortment in the information condition being viewed as more suitable.

ANOVA's were also performed to compare condition-specific assortment evaluations to the baseline assortment in the control condition. Table 5.4 shows that respondents perceived the caloric values of the products in the assortment to be clearer when the information intervention was implemented, compared to the control condition. Additionally, compared to the control condition, respondents perceived assortment pricing to be slightly less reasonable when the price 
intervention was implemented. Evaluations of diversity and completeness of the assortment in the control condition did not differ from the assortment diversity ratings in the availability condition and the assortment completeness ratings in the restriction condition.

A linear regression analysis of choice evaluations on assortment evaluations was then performed. Assortment attractiveness positively influenced choice satisfaction, while perceived assortment healthiness and assortment suitability in schools and universities did not influence the level of frustration when making choices and the subsequent (dis)satisfaction with the choices (Table 5.7).

Table 5.7: Linear regression analysis of choice evaluations on assortment evaluations

\begin{tabular}{lcc}
\hline & Frustration & Dissatisfaction \\
\cline { 2 - 3 } & $\boldsymbol{\beta}$ & \multicolumn{1}{c}{$\boldsymbol{\beta}$} \\
\hline Assortment evaluations & & \\
Attractiveness & -0.140 & $-0.286^{* * *}$ \\
Healthiness & 0.121 & -0.114 \\
Suitability & 0.000 & -0.096 \\
& & \\
$\mathrm{R}^{2}$ & 0.030 & 0.130 \\
(Adjusted) & $(0.015)$ & $(0.117)$ \\
\hline$* 0<05^{* *} p<0.01 * * * p<0.001$ &
\end{tabular}

${ }^{*} p<0.05,{ }^{* *} p<0.01, * * * p<0.001$

Relation between low-calorie snack choices, intervention acceptance, and choice evaluations

To explore whether the three conditions for long-term intervention effectiveness were related, additional logistic regression and correlation analyses were performed. Both intervention acceptance and choice evaluations were not related to low-calorie choices. Additionally, no correlation was found between intervention acceptance and choice evaluations. This suggests that the behavioural, cognitive, and affective outcomes of intervention strategies are not necessarily interrelated.

\section{Evaluations of real-life versus hypothetical interventions}

ANOVA's were performed to examine whether interventions were evaluated differently by respondents who experienced the intervention in a realistic setting (in Stage 1), compared to respondents who evaluated it as a hypothetical intervention (in Stage 2). The mean differences in Table 5.8 show that all types of interventions were accepted more when they were actually experienced, compared to when they 
were evaluated as hypothetical interventions. The opposite was true for perceived intrusiveness; availability, price, and restriction interventions were perceived to be less intrusive when they were actually experienced.

Respondents in the price condition perceived price increases for high-calorie choices to be less effective than respondents in other conditions, but simultaneously they perceived these increases to be fairer as well. Additionally, elimination of half of the most high-caloric products was perceived as fairer by those in the restriction condition, compared to other respondents. Respondents in the availability condition perceived the increase of facings for low-calorie products to be more effective on a societal level than those in other conditions.

Table 5.8: Mean differences between evaluations of hypothetical and real-life interventions

\begin{tabular}{|c|c|c|c|c|c|c|c|c|}
\hline & \multicolumn{8}{|c|}{ Intervention type } \\
\hline & \multicolumn{2}{|c|}{ Information } & \multicolumn{2}{|c|}{ Availability } & \multicolumn{2}{|c|}{ Price } & \multicolumn{2}{|c|}{ Restriction } \\
\hline & Mean & SE & Mean & SD & Mean & SD & Mean & SD \\
\hline \multicolumn{9}{|l|}{ Intervention acceptance } \\
\hline Acceptance $^{1}$ & $-0.61 *$ & 0.28 & $-1.04 * * *$ & 0.25 & $-0.89 * *$ & 0.31 & $-1.22 * * *$ & 0.31 \\
\hline Perceived & & & & & & & & \\
\hline personal effectiveness ${ }^{1}$ & -0.23 & 0.29 & -0.47 & 0.29 & $1.00 * * *$ & 0.26 & -0.04 & 0.25 \\
\hline Perceived & & & & & & & & \\
\hline societal effectiveness ${ }^{1}$ & -0.33 & 0.24 & $-0.87 * *$ & 0.27 & $0.61 * *$ & 0.23 & 0.07 & 0.21 \\
\hline Perceived fairness ${ }^{1}$ & 0.08 & 0.23 & -0.39 & 0.23 & $-0.90 * *$ & 0.30 & $-0.67 *$ & 0.31 \\
\hline Perceived intrusiveness ${ }^{2}$ & 0.24 & 0.14 & $0.56^{* *}$ & 0.17 & $0.60 * *$ & 0.20 & $0.33^{*}$ & 0.16 \\
\hline
\end{tabular}

${ }^{1}$ Range 1-7; ${ }^{2}$ Range 1-5

${ }^{*} p<0.05,{ }^{* *} p<0.01,{ }^{* * *} p<0.001$ 


\subsection{Discussion}

The present study examined the effects of intervention intrusiveness on effectiveness, acceptance, and choice evaluations in relation to vending-machine interventions. Four types of interventions were implemented: providing calorie information, guiding choice through assortment incentives, guiding choice through price disincentives, and restricting choice. By employing a variety of incentivealigned interventions in a realistic setting, the present study contributes relevant insights to existing research on behavioural, cognitive, and affective responses to intervention strategies for healthy food choices.

We found that restricting the number of high-calorie options in the assortment was more effective in stimulating people to choose healthier products from the vending machine than providing calorie information or doing nothing. More specifically, 39\% of all respondents selected a low-calorie snack from an assortment with one half low-calorie options, while $78 \%$ of all respondents selected a low-calorie snack from an assortment with two-third low-calorie options. Both groups considered the presented assortment as equally attractive. This is in line with other research that indicates superior effectiveness of interventions that change the market, compared to interventions that support informed choice (Brambila-Macias et al., 2011). In addition, this result underlines the need and supports the widespread call for more intrusive measures to effectively combat the obesity epidemic.

Contrary to earlier research in which intrusive interventions are accepted less (Diepeveen et al., 2013), we found acceptance to be similar across intervention types. In the case of choice restriction and price disincentives this is an important finding, particularly because restriction and price increases were identified as the least accepted governmental interventions in earlier research (Bos et al., 2015a).

Additionally, levels of frustration when making choices and dissatisfaction with these choices were not higher when people were restricted in their options of high-calorie choices, compared to when they were not. This could be due to the fact that, despite the omission of half of the high-calorie options, there were still one or more options present for the different types of products (i.e. candy bars, cookies, fruit- and vegetable snacks). Therefore respondents may have perceived the assortment to remain sufficiently varied in choice options (Mogilner, Rudnick, \& Iyengar, 2008). 
The overall finding that restricting high-calorie options not only leads to the highest proportion of healthier choices, but is also equally accepted as providing information and increasing availability, and does not increase frustration and dissatisfaction, provides a promising avenue for the design of future vending machine interventions. The validity of this finding is strengthened by the fact that personal relevance was emphasized during the study by explicitly telling respondents that implementation of the interventions in the near future was quite certain.

Interventions were perceived as more effective and fair, and were accepted more when they were actually experienced, compared to when they were presented as hypothetical strategies on paper. This positive difference in evaluations of interventions could be due to the fact that the interventions in the realistic setting were perceived as more absolute (i.e. more certain to coming into effect). Higher levels of intervention absoluteness are known to increase support for intrusive interventions, because it stimulates people to rationalize the new situation to avoid cognitive dissonance (Laurin et al., 2012). More clarity on which psychological process can explain these differences is important, as it provides guidelines for the communication and implementation of future intervention strategies.

Contrary to expectations based on Rothschild's Motivation-Opportunity-Ability framework, there is no evidence for an intervention-barrier fit in terms of intervention effectiveness and acceptance. We did, however, find a direct link between barriers for choosing low-calorie snacks on the one hand and intervention effectiveness and intervention evaluations on the other hand. More specifically, respondents who reported having no barriers chose low-calorie snacks more often, perceived interventions to be more effective and fair, and accepted interventions more than respondents who were not motivated to choose low-calorie snacks. This is in line with Bos et al. (2015a, 2015b), who found that barriers for choosing lowcalorie snacks play an important role in explaining both snack choice behaviour and acceptance of snack choice interventions.

Across the different intervention types, there was no relation between short-term effectiveness, acceptance, and choice evaluations. This indicates that the influence of intervention intrusiveness expresses itself differently on behavioural, cognitive, and affective levels. It is, however, plausible that these conditions for long-term effectiveness become more related when interventions are extremely intrusive. Prohibition of high-calorie snacks, for instance, would likely lead to perceptions of 
unfairness and low acceptance due to restriction of the freedom of choice as well as frustration and dissatisfaction with choices due to low assortment attractiveness.

\section{Limitations and future research}

Because excess energy intake is the main driver of the obesity epidemic (Bleich et al., 2008), healthiness of the snack foods and beverages was derived from the caloric values of the products in the vending machines. Other characteristics than calorie content can also be of importance in light of responsible dietary behaviours. Snack foods and beverages with added vitamins, for instance, can help to diminish vitamin deficiencies. Whether the results of the present study are generalizable to other characteristics that can be used to operationalize healthiness is therefore valuable information.

The present study purposely selected a student population because vending machine density is particularly high in universities. It is possible that this specific population accepts vending machine interventions more than other populations in other situations (e.g. children in high schools). Some studies have investigated the effectiveness of interventions in relation to specific populations (Doak et al., 2006) and specific locations (Lowe et al., 2010; Maes et al., 2012). With regard to acceptance of interventions, however, this research is lacking. To examine whether the present study's findings regarding acceptance are generalizable to other contexts, a next step is to examine how different populations and different choice situations influence intervention acceptance.

Respondents paid for the snacks with the money they received for completing the non-related task, therefore there is a possibility that they perceived paying for the products to be a reduction of their gain, rather than a loss of their money. This could lead to different buying behaviour. As no respondent mentioned the "type" of money as a reason for choosing the products they chose, however, there is little reason to believe that respondents' buying behaviour was influenced by using participation money.

The present study investigated snack choices on a single occasion. It would be valuable to examine whether effectiveness of the different types of interventions changes over time. Kocken et al. (2012) executed such a study in a number of schools for six months in which they first increased availability of lower-calorie products, then added labelling to the products, and finally also reduced prices of low-calorie products. They found that students only make healthier choices when all three 
interventions were implemented. For future research, more types of interventions could be used and effectiveness should be assessed for both separate interventions and combinations of interventions.

Additionally, across these interventions, one should explore whether people will keep using the vending machines to obtain their snack foods and beverages. It is possible that, due to the interventions, people will substitute their points-ofpurchase, as financial considerations, availability of preferred products, and other point-of-purchase characteristics are known to influence point-of-purchase choice (Krukowski et al., 2012).

Overall, the findings provide intervention opportunities not only for governments, but also for other stakeholders in the food domain that are important allies in the combat against obesity, such as food manufacturers and the food service industry. Large food manufacturers could intervene themselves by collectively decreasing the number of high-calorie products in their product portfolios, for instance as an implementation of corporate social responsibility (Shnayder, Van Rijnsoever, \& Hekkert, 2015), without encountering negative effects on their sales. 

GENERAL DISCUSSION 
The present dissertation found its origin in the observations that a multitude of intervention strategies with various levels of intrusiveness have been largely ineffective in lowering obesity rates (Brambila-Macias et al., 2011; Capacci et al., 2012); that a lack of acceptance of intervention strategies may critically hamper their effectiveness (Diepeveen et al., 2013; Kelly et al., 2009); that there exists limited knowledge on why the public does or does not accept such interventions (Mazzocchi et al., 2014); and that tailoring interventions to people's barriers for behaviour maximizes effectiveness (Andreasen, 2002; Gordon et al., 2006; Rothschild, 1999).

By acknowledging that excessive calorie intake is the main cause of overweight and obesity (Popkin \& Duffey, 2010; Shelley, 2012) this thesis purposely focused on interventions that aim to stimulate low-calorie food choices (which, for consistency purposes, are called healthy food choices for the rest of the discussion).

Overall, the aim of the present thesis was to understand the process underlying consumer acceptance of intervention strategies for healthy food choices. The research specifically focused on the influence of intervention characteristics and perceived barriers for behaviour within this process. To accomplish the overall aim, three research questions were addressed: 1) What are the drivers of consumer acceptance of intervention strategies for healthy food choices?; 2) How do consumers' barriers for healthy food choices influence both food choices in real-life and intervention acceptance?; 3) How does intervention intrusiveness (i.e. limitations to the freedom of choice induced by interventions) influence acceptance and effectiveness of intervention strategies for healthy food choices? This chapter gives an overview of the answers to the research questions and the implications for science and practice. Furthermore, limitations and suggestions for further research are provided.

\subsection{Summary and conclusions}

\section{Drivers of acceptance of intervention strategies for healthy food choices}

To explore the concept of consumer acceptance in light of intervention strategies for healthy food choices, educational, marketing, and legal interventions were used as reference points to elicit drivers that are associated with acceptance. Chapter 2 identified general beliefs about the obesity problem and intervention-specific beliefs as the main drivers of intervention acceptance in the food domain. General beliefs concerned both people's ideas about the magnitude of the obesity problem and the 
responsibility for food choices that consumers make. This is in line with other research on obesity prevention, which found that acceptance is influenced by whether people attribute being obese to individual or societal causes (Barry et al., 2009; Chambers \& Traill, 2011; Hilbert et al., 2007; Mazzocchi et al., 2014; Pearl \& Lebowitz, 2014). Intervention-specific beliefs, on the other hand, comprised people's perceptions of whether an intervention will be effective, both personally and societally, in stimulating healthy food choices (perceived effectiveness) as well as whether the intervention is a fair way of stimulating healthier choices (perceived fairness). To assess effectiveness, people relied on a variety of sources: some used information on societal effectiveness of interventions in other domains (e.g. tobacco and alcohol), others looked at how well interventions responded to perceived barriers for healthy choices. To indicate perceptions of fairness, people considered negative consequences of interventions: some were concerned about adverse intervention effects on specific groups while others worried about losing freedom of choice. Such concerns about the loss of choice autonomy are in line with the ethical issues regarding prevention of overweight and obesity that are identified by experts (Ten Have et al., 2011).

By examining drivers of (non)acceptance of interventions for healthy food choices and their underlying aspects, Chapter $\mathbf{2}$ helps policymakers to anticipate and address low support for existing and novel interventions. Furthermore, by identifying both general beliefs and intervention-specific beliefs as drivers of acceptance, this chapter replicated the process of intervention acceptance from the transport policy domain (Eriksson et al., 2008). This suggests that the process of intervention acceptance is, at least on a conceptual level, generalizable across application domains.

\section{Barriers for healthy food choices and their relation to food choices in real-life} To be able to intervene effectively and thus maximize behaviour change, knowledge on what prevents people from choosing healthy foods is needed (S. D. French et al., 2012). Rothschild's Motivation-Opportunity-Ability (MOA) framework (1999) states that people's barriers for behaviour can be both individual (i.e. motivation and ability to choose healthy foods) and environmental (i.e. opportunity to choose healthy foods) in nature (Brug, 2008; Van Trijp et al., 2005), and that intervention strategies based on education, marketing, and law need to be tailored to these barriers to maximize effectiveness. Chapter 3 employed the MOA framework and investigated which barriers people perceive to have and how these are related to real-life food and beverage choices. We found that motivation to choose healthy foods was associated strongest with real-life choices, with higher levels of motivation being 
related to higher proportions of healthy choices. This is in line with earlier research (Dibsdall et al., 2003; Hearty et al., 2007). Perceived opportunity and ability to choose healthy foods were associated less strongly with real-life choices and this only applied to snack foods, with higher levels of opportunity and ability being related to higher proportions of healthy snack food choices. These findings imply that, of the three barrier types, increasing motivation will yield the largest effect in improving healthy food choice behaviour, which has been echoed in other research (Baranowski et al., 2003).

While the MOA framework theorizes eight possible barrier profiles, we identified three distinct barrier profiles: those who perceive to have no barriers (no-barrier profile), those who perceive to lack opportunity (lack-of-opportunity profile), and those who report not being motivated (lack-of-motivation profile). These barrier profiles existed both in the snack food and the beverage context, which implies robustness across product categories. Other research on barriers to choose specific food products, such as seafood and fruit and vegetables, also suggests that similar barrier profiles exist (D. Birch \& Lawley, 2012; Trudeau, Kristal, Li, \& Patterson, 1998).

By showing how individual-level and environmental-level barriers for healthy choices are related to real-life food choice behaviour, Chapter 3 helps us to understand the drivers of unhealthy food choice behaviour that need to be overcome by interventions. Additionally, by identifying three dominant barrier profiles based on the MOA framework, we provide directions for the tailoring of intervention strategies.

\section{Influence of intervention intrusiveness on acceptance and effectiveness}

To further uncover the process underlying acceptance, both the effect of intervention intrusiveness (i.e. limitations to the freedom of choice induced by interventions) on acceptance and the role that the perceived personal- and societal effectiveness and the perceived fairness play in this relation were examined. Chapter 4 presented people with descriptions of hypothetical interventions and showed that the 'objective' level of intrusiveness, as presented by Nuffield's intervention ladder (2007) on an ordinal scale, influences acceptance of intervention strategies. Acceptance of less intrusive interventions, such as calorie information provision, was higher than those of more intrusive interventions, such as elimination of unhealthy choices, which was in line with findings from a systematic review on acceptance of government interventions to change health-related behaviours (Diepeveen et al., 2013). The effect of intrusiveness on acceptance was mediated by both the 
perceived effectiveness and the perceived fairness of interventions: the more people perceived an intervention to be effective and fair, the more they accepted it. This was in line with expectations based on Chapter $\mathbf{2}$ and replicated results from the transport domain (Eriksson et al., 2008).

We subsequently tested whether the effect of intervention intrusiveness on acceptance of interventions that are presented in a hypothetical manner (Chapter 4) was generalizable to acceptance of interventions that are implemented in a more realistic setting. Chapter $\mathbf{5}$ indicated that when people actually experienced interventions in a realistic setting, acceptance did not differ across different levels of intervention intrusiveness.

To identify differences in objective intrusiveness (as classified by Nuffield's intervention ladder) and people's perceptions of intrusiveness, the perceived intrusiveness of the different types of interventions were also obtained. Perceptions of more intrusive interventions aligned with the objective intrusiveness (e.g. elimination of unhealthy choices was perceived as more intrusive than increasing the number of facings for healthy products). Interventions on the lower end of the intrusiveness spectrum, however, were perceived to be similarly intrusive, even though objectively they differed (e.g. increasing the number of facings for healthy products was not perceived as more intrusive than provision of calorie information). Perceptions of intrusiveness díd influence acceptance; lower perceptions of intrusiveness were associated with higher acceptance. This relation was mediated only by the perceived fairness, and not by the perceived effectiveness of interventions, as perceptions of effectiveness did not differ across intervention types.

By comparing hypothetical and realistic ways of presenting interventions, Chapters $\mathbf{4}$ and $\mathbf{5}$ shed light on the effect of intervention absoluteness on the relation between (objective) intrusiveness and consumer acceptance.

Both for hypothetical (Chapter 4 ) and realistic (Chapter 5 ) interventions, we did not find an interaction between barrier profile and intervention type on acceptance, which we hypothesized based on the MOA framework. Instead, we found that, for all the different levels of intrusiveness, people with a no-barrier profile or a lack-ofopportunity profile perceived interventions to be more effective, more fair, and more acceptable than those with a lack-of-motivation profile. This can be explained by the fact that the main difference between the no-barrier profile and the lack-of- 
opportunity barrier profiles on the one hand and the lack-of-motivation profile on the other hand, was the level of motivation to choose healthy foods, which is known to strongly influence intervention acceptance (Holmila, Mustonen, Österberg, \& Raitasalo, 2009; Walsh, Paul, Tzelepis, Stojanovski, \& Tang, 2008).

When zooming in at the effect of intrusiveness on effectiveness of interventions, it became clear that the implementation of the most intrusive intervention, eliminating $50 \%$ of unhealthy choices, led to higher proportions of healthy choices than the implementation of the least intrusive intervention (providing calorie information through labelling) and no intervention at all, which is in line with earlier research (Giesen et al., 2011). Coupled with the finding that acceptance did not differ between intervention types, elimination of unhealthy choices therefore seems to be a promising way to stimulate healthy choices.

By experimentally testing acceptance and effectiveness of four different types of food choice interventions, Chapter 5 provides insights in the relation between cognitive and behavioural reactions elicited by interventions in a realistic setting.

\subsection{Scientific implications}

\section{Process of acceptance of intervention strategies for healthy food choices}

Acknowledging that a lack of acceptance may interfere with intervention effectiveness, the present thesis examined the process underlying consumer acceptance of intervention strategies for healthy food choices. The studies show that intervention characteristics, perceptions of intervention effectiveness and fairness, and barriers for healthy food choices influence acceptance.

With respect to intervention characteristics, we found that the influence of intervention intrusiveness on acceptance is inconclusive. Where we did find an effect of intrusiveness on acceptance of hypothetical interventions that were presented on a computer screen (Chapter 4), we did not find this effect for interventions implemented in a vending machine in a realistic setting (Chapter 5 ). This suggests that how interventions are presented influences whether intervention intrusiveness affects acceptance. Laurin et al. (2012) provide an explanation for this discrepancy by demonstrating that acceptance of intrusive intervention increases as absoluteness (i.e. certainty of coming into effect) of these interventions increases. It is likely that interventions that are actually experienced in a realistic setting are 
perceived as more absolute (i.e. more certain to be implemented) than hypothetical interventions encountered on a computer screen.

The present thesis furthermore showed that objective intervention intrusiveness, as classified on Nuffield's ordinal intervention ladder (2007), does not necessarily align with people's perceptions of intrusiveness. We found that, when presented in a realistic setting, people perceive less intrusive interventions to be equally (un)intrusive (even though they differ on the Nuffield ladder), while they do perceive differences between interventions on the higher end of the intrusiveness spectrum. This finding implies the importance of also obtaining people's perceptions of intrusiveness in future research on intervention acceptance, particularly because these perceptions predicted intervention acceptance better than objective intrusiveness (Chapter 5).

The effect of tailoring interventions to barriers for healthy food choices on consumer acceptance and intervention effectiveness

Social marketing stresses the necessity to understand people's barriers for behaviour to enable 'tailoring' of interventions and maximize effectiveness (Gordon et al., 2006; Kolodinsky \& Reynolds, 2009). Employing the MOA framework, we demonstrated that the presence of barriers regarding the motivation, the opportunity, and the ability to choose healthy foods is related to unhealthy food choice behaviour. This implies that if interventions are successful in diminishing barriers, proportions of healthy food choices will increase.

To successfully diminish these barriers, the MOA framework theorizes that interventions need to fit the barriers that people have (i.e. responsiveness to interventions depends on the barrier profile). However, we found no evidence of differences in responsiveness between barrier profiles in terms of intervention evaluations. It should be taken into account, however, that we only found three dominant barrier profiles out of the hypothesized eight profiles based on the MOA framework. It is possible that when more (diverse) barrier profiles are identified, responsiveness to interventions in terms of acceptance does differ, which has been found in earlier research (Geeroms, Verbeke, \& Van Kenhove, 2008b).

Additionally, we found no evidence of differences in responsiveness between barrier profiles in terms of intervention effectiveness. This finding implies that, keeping in mind the conditions for segmentation to be an effective tool (Kotler \& Keller, 2006), using barrier profiles to optimize intervention effectiveness only has limited 
usefulness. It should be noted, however, that we only compared two barrier profiles and that we only used a single choice occasion to assess responsiveness in terms of intervention effectiveness. Differences in responsiveness to interventions could emerge when more barrier profiles are identified and when a multitude of food choices over a longer period of time are assessed. Future studies on the effectiveness of food choice interventions are therefore encouraged to employ the MOA framework and take such a prospective approach to see whether changes in responsiveness occur.

\section{Relation between intervention acceptance and effectiveness}

To date, research on outcomes of interventions has mainly focused on either people's evaluations of interventions (Berezowska et al., 2014; Diepeveen et al., 2013; Promberger et al., 2011) or the behavioural effects of interventions (Ammerman, Lindquist, Lohr, \& Hersey, 2002; Capacci et al., 2012; Pomerleau, Lock, Knai, \& McKee, 2005; Verweij, Coffeng, van Mechelen, \& Proper, 2011). Therefore information on the relation between acceptance and effectiveness is scarce. The present thesis makes a unique contribution to both types of research by studying the relation between acceptance and effectiveness of food choice interventions in an experimental setting. We found no evidence of a relation between acceptance and (short-term) effectiveness, because acceptance did not differ between the types of interventions. This finding should be put into perspective, as it applies to a single choice occasion in a vending machine context. Whether the relation between acceptance and effectiveness does exist in contexts other than vending machines, and over longer periods of time, needs to be examined.

\subsection{Practical implications}

The findings that have been presented in the present thesis have a number of practical implications for policy makers in the food domain. The results may also provide food manufacturers with opportunities to stimulate healthy food choices, particularly because we found that interventions that are initiated by the food industry are accepted more than those by the government. The implications concern the design of intervention strategies, the anticipation of resistance towards interventions, and the implementation of more intrusive interventions. 


\section{Design of intervention strategies}

In terms of developing (novel) intervention strategies, the present thesis provides implications based on the concerns that people have regarding the fairness and effectiveness of interventions. In the case of fairness of taxing unhealthy products, for instance, people expressed concerns that governments would only implement such interventions to generate extra income. To counteract these concerns of unfairness, the tax revenue generated by such an intervention could be used for subsidizing healthy products or physical activity programs, thereby increasing perceptions of fairness and thus acceptance of tax interventions. This effect of tax allocation on acceptance has been found in the case of energy policies (Steg, Dreijerink, \& Abrahamse, 2006).

Additionally, when people think an intervention will not work, influencing their perceptions by providing evidence for effectiveness can help to increase acceptance. Communicating positive expectations about the effectiveness of interventions, for instance, has shown to increase acceptance of these interventions (Pechey et al., 2014; Promberger et al., 2012).

\section{Anticipation of resistance towards interventions}

The results of the vending machine experiment (Chapter 5) suggest that implementing interventions that limit the proportion of unhealthy choices would be the most effective way to stimulate healthy food choices, and that this intervention would be accepted by a majority. In the event that such an intervention would be widely implemented, however, there is a possibility that people who feel negatively affected by the intervention organize against these policies. Such resistance can have large consequences for the implementation and continuation of interventions. Schaller (2010) describes how a relatively small group that would be negatively affected by a proposed congestion tax prevented it from coming into effect, despite the fact that this proposal had gained widespread public support. Monitoring resistance among groups that are likely to be most affected by proposed interventions is therefore recommended.

\section{Implementation of more intrusive interventions}

By demonstrating that acceptance of interventions in a realistic setting can be similarly high across levels of intrusiveness, the present thesis also provides an overall incentive to governments and food manufacturers to 'be bold' and implement more intrusive interventions to stimulate healthy food choices. Even though a risk of reactance is looming, this risk is worth taking, particularly because 
public health experts agree that more intrusive interventions are needed to halt the obesity epidemic while literature also shows that they are more effective than less intrusive interventions (Nestle \& Jacobson, 2000; Swinburn et al., 2011).

\subsection{Limitations and future research}

The present thesis investigated the process of consumer acceptance of interventions for healthy food choices. Limitations to the research that was presented, and suggestions for future research to address them, are related to potential changes in acceptance and effectiveness over time and within contexts, and potential other factors that influence consumers' acceptance of interventions.

\section{Acceptance and effectiveness over time and in different contexts}

We examined consumer acceptance of interventions in two types of settings: hypothetical, in which interventions were merely described, and realistic, in which interventions were actually experienced. This provided information on differences in consumer acceptance along two different stages before implementation. To map how consumer acceptance of a specific intervention manifests itself along all stages of implementation, knowledge on consumer acceptance after implementation is also needed. Nilsson, Schuitema, Jakobsson, Martinsson, and Thorson (2016) provided a blueprint for prospectively examining this process by investigating people's acceptance of congestion taxes before and after implementation. They found that acceptance of the congestion tax had increased after it was implemented, which is in line with rationalization theory (Kay et al., 2002). Future research on consumer acceptance of food choice interventions is encouraged to adopt a similar prospective approach.

To investigate intervention effectiveness, the present thesis employed an experiment in a vending machine context, in which people were asked to choose a snack food and a beverage. Although such choices provide information on people's responsiveness to specific intervention types in a particular context, they do not tell how effective interventions will be over a longer period of time, nor do they ensure that the interventions will be similarly (in)effective in other settings. Kocken et al. (2012) have incorporated these concepts by employing a school-based randomized controlled trial in 13 experimental and 15 control schools. They investigated the long-term effect of three intervention strategies (increasing availability of healthy products, labelling, and price reduction of healthy products) on vending machine 
choices and found that students only make healthier choices when the three interventions are simultaneously implemented. Future studies on intervention effectiveness are encouraged to take both effectiveness over time as well as across contexts, into account when designing research.

\section{Additional factors that potentially influence intervention acceptance}

A factor that the present thesis did not include, but which has shown to be important for tax acceptance, is the distribution of tax revenues that are obtained through the implementation of tax interventions. Allocating tax revenues within the same domain leads to higher intervention acceptance than when tax revenues are added to general funds (Steg et al., 2006). Considering that tax measures are among the least accepted interventions in the present thesis, investigating whether tax acceptance increases when revenues are allocated within the food domain, for instance through subsidizing fruit and vegetables, will provide relevant information for the development of tax interventions.

\subsection{Final conclusion}

The present thesis shows that people mainly rely on perceptions of interventions' effectiveness and fairness when accepting or not accepting intervention strategies for healthy food choices. Furthermore, acceptance of intrusive interventions is influenced by the way they are presented: people accept intrusive interventions more when they are presented in a realistic setting than when they are described as a hypothetical strategy. These insights provide implications for policy makers, both in terms of developing new intervention strategies and the communication of these strategies.

The present thesis demonstrates that people have both individual-level and environmental-level barriers for choosing healthy foods, and that these are related to actual food choice behaviour. Additionally, three dominant barrier profiles for healthy food choices are identified; the no-barrier, lack-of-opportunity, and lack-ofmotivation profiles. By showing that these barrier profiles differ in perceptions of effectiveness, fairness, and acceptability across all levels of intrusiveness, the present thesis indicates the usefulness of taking barriers for behaviour into account when designing novel interventions. 
By providing evidence that more intrusive interventions lead to more healthy choices, the present thesis provides directions to break the 'lock-in' within the current food system and also echoes the calls of researchers in the food domain and public health experts, who both stress the necessity of more radical interventions to combat the obesity problem effectively. 


\section{REFERENCES}

Ajzen, I. (1991). Theories of cognitive self-regulation: the theory of planned behavior. Organizational Behavior and Human Decision Processes, 50(2), 179-211. doi:10.1016/0749-5978(91)90020-T

Ajzen, I., \& Timko, C. (1986). Correspondence between health attitudes and behavior. Basic and Applied Social Psychology, 7(4), 259-276. doi:10.1207/s15324834basp0704_2

Ammerman, A. S., Lindquist, C. H., Lohr, K. N., \& Hersey, J. (2002). The efficacy of behavioral interventions to modify dietary fat and fruit and vegetable intake: a review of the evidence. Preventive Medicine, 35(1), 25-41.

Andreasen, A. R. (2002). Marketing social marketing in the social change marketplace. Journal of Public Policy \& Marketing, 21(1), 3-13.

Andrieu, E., Darmon, N., \& Drewnowski, A. (2006). Low-cost diets: more energy, fewer nutrients. European Journal of Clinical Nutrition, 60(3), 434-436.

Avena, N. M., Rada, P., \& Hoebel, B. G. (2008). Evidence for sugar addiction: behavioral and neurochemical effects of intermittent, excessive sugar intake. Neuroscience \& Biobehavioral Reviews, 32(1), 20-39.

Bandini, L. G., Vu, D., Must, A., Cyr, H., Goldberg, A., \& Dietz, W. H. (1999). Comparison of high-calorie, low-nutrient-dense food consumption among obese and non-obese adolescents. Obesity Research, 7(5), 438-443. doi:10.1002/j.1550-8528.1999.tb00431.x

Baranowski, T., Cullen, K. W., Nicklas, T., Thompson, D., \& Baranowski, J. (2003). Are current health behavioral change models helpful in guiding prevention of weight gain efforts? Obesity Research, 11, 23S-43S. doi:10.1038/oby.2003.222

Baron, J. (2009). Belief overkill in political judgments. Social Science Research Network. doi:10.2139/ssrn.1427862

Baron, R. M., \& Kenny, D. A. (1986). The moderator mediator variable distinction in social psychological-research - conceptual, strategic, and statistical considerations. Journal of personality and social psychology, 51(6), 11731182. doi:10.1037/0022-3514.51.6.1173

Barry, C. L., Brescoll, V. L., Brownell, K. D., \& Schlesinger, M. (2009). Obesity metaphors: How beliefs about the causes of obesity affect support for public policy. Milbank Quarterly, 87(1), 7-47. doi:10.1111/j.14680009.2009.00546.x

Bellisari, A. (2008). Evolutionary origins of obesity. Obesity reviews, 9(2), 165-180.

Berezowska, A., Fischer, A. R., Ronteltap, A., Kuznesof, S., Macready, A., Fallaize, R., \& Van Trijp, H. C. (2014). Understanding consumer evaluations of personalised nutrition services in terms of the privacy calculus: a qualitative study. Public health genomics, 17(3), 127-140. 
Bingham, S. A., Luben, R., Welch, A., Wareham, N., Khaw, K.-T., \& Day, N. (2003). Are imprecise methods obscuring a relation between fat and breast cancer? The Lancet, 362(9379), 212-214. doi:10.1016/S01406736(03)13913-X

Birch, D., \& Lawley, M. (2012). Buying seafood: Understanding barriers to purchase across consumption segments. Food Quality and Preference, 26(1), 12-21.

Birch, L. L. (1999). Development of food preferences. Annual Review of Nutrition, 19(1), 41-62. doi:10.1146/annurev.nutr.19.1.41

Bland, J. M., \& Altman, D. G. (2000). The odds ratio. BMJ, 320(7247), 1468. doi:10.1136/bmj.320.7247.1468

Bleich, S., Cutler, D., Murray, C., \& Adams, A. (2008). Why is the developed world obese? Annual review of public health (Vol. 29, pp. 273-295). Palo Alto: Annual Reviews.

Boehmer, T. K., Hoehner, C. M., Deshpande, A. D., Ramirez, L. K. B., \& Brownson, R. C. (2007). Perceived and observed neighborhood indicators of obesity among urban adults. International Journal of Obesity, 31(6), 968-977. doi:10.1038/sj.ijo.0803531

Boles, M., Adams, A., Gredler, A., \& Manhas, S. (2014). Ability of a mass media campaign to influence knowledge, attitudes, and behaviors about sugary drinks and obesity. Preventive Medicine(0), S40-S45. doi:10.1016/j.ypmed.2014.07.023

Bos, C., Van der Lans, I. A., Van Rijnsoever, F. J., \& Van Trijp, H. C. M. (2013). Understanding consumer acceptance of intervention strategies for healthy food choices: a qualitative study. BMC public health, 13, 1073. doi:10.1186/1471-2458-13-1073

Bos, C., Van der Lans, I. A., Van Rijnsoever, F. J., \& Van Trijp, H. C. M. (2015a). Consumer acceptance of population-level intervention strategies for healthy food choices: The role of perceived effectiveness and perceived fairness. Nutrients, 7(9), 7842-7862. doi:10.3390/nu7095370

Bos, C., Van der Lans, I. A., Van Rijnsoever, F. J., \& Van Trijp, H. C. M. (2015b). Heterogeneity in barriers regarding the motivation, the opportunity and the ability to choose low-calorie snack foods and beverages: Associations with real-life choices. Public Health Nutrition, 19(9), 1584-1597. doi:10.1017/S1368980015002517

Bowling, A. (2005). Mode of questionnaire administration can have serious effects on data quality. Journal of Public Health, 27(3), 281-291. doi:10.1093/pubmed/fdi031

Brambila-Macias, J., Shankar, B., Capacci, S., Mazzocchi, M., Perez-Cueto, F. J. A., Verbeke, W., \& Trail, W. B. (2011). Policy interventions to promote healthy eating: A review of what works, what does not, and what is promising. Food and Nutrition Bulletin, 32(4), 365-375.

Brehm, J. W. (1966). A theory of psychological reactance. Oxford England: Academic Press. 
Brownell, K. D., Parley, T., Willett, W. C., Popkin, B. M., Chaloupka, F. J., Thompson, J. W., \& Ludwig, D. S. (2009). The public health and economic benefits of taxing sugar-sweetened beverages. New England Journal of Medicine, 361(16), 1599-1605.

Brug, J. (2008). Determinants of healthy eating: motivation, abilities and environmental opportunities. Family Practice, 25, I50-I55. doi:10.1093/fampra/cmn063

Burgoine, T., Lake, A. A., Stamp, E., Alvanides, S., Mathers, J. C., \& Adamson, A. J. (2009). Changing foodscapes 1980-2000, using the ASH30 Study. Appetite, 53(2), 157-165. doi:10.1016/j.appet.2009.05.012

Byers, T. (2001). Food frequency dietary assessment: how bad is good enough? American Journal of Epidemiology, 154(12), 1087-1088. doi:10.1093/aje/154.12.1087

Cade, J., Thompson, R., Burley, V., \& Warm, D. (2002). Development, validation and utilisation of food-frequency questionnaires - a review. Public Health Nutrition, 5(4), 567-587. doi:10.1079/phn2001318

Capacci, S., Mazzocchi, M., Shankar, B., Macias, J. B., Verbeke, W., Perez-Cueto, F. J. A., ... Traill, W. B. (2012). Policies to promote healthy eating in Europe: a structured review of policies and their effectiveness. Nutrition Reviews, 70(3), 188-200. doi:10.1111/j.1753-4887.2011.00442.x

Catlin, T. K., Simoes, E. J., \& Brownson, R. C. (2003). Environmental and policy factors associated with overweight among adults in Missouri. [American Journal of Health Promotion]. American Journal of Health Promotion, 17(4), 249-258. doi:10.4278/0890-1171-17.4.249

Chambers, S. A., \& Traill, W. B. (2011). What the UK public believes causes obesity, and what they want to do about it: A cross-sectional study. Journal of Public Health Policy, 32(4), 430-444. doi:10.1057/jphp.2011.45

Charrad, M. G., N; Boiteau, V; Niknafs, A. (2014). Nbclust: An R package for determining the relevant number of clusters in a data set. Journal of Statistical Software, 61(6).

Chouinard, H. H., Davis, D. E., LaFrance, J. T., \& Perloff, J. M. (2007). Fat taxes: Big money for small change. Paper presented at the Forum for Health Economics \& Policy.

Cohen, D. A., Sturm, R., Scott, M., Farley, T. A., \& Bluthenthal, R. (2010). Not enough fruit and vegetables or too many cookies, candies, salty snacks, and soft drinks? Public Health Reports, 125(1), 88-95.

Conner, M., Norman, P., \& Bell, R. (2002). The theory of planned behavior and healthy eating. Health Psychology, 21(2), 194-201.

Crites, S. L., Fabrigar, L. R., \& Petty, R. E. (1994). Measuring the affective and cognitive properties of attitudes - conceptual and methodological issues. Personality and Social Psychology Bulletin, 20(6), 619-634. doi:10.1177/0146167294206001

Dayan, E., \& Bar-Hillel, M. (2011). Nudge to nobesity II: Menu positions influence food orders. Judgment and Decision Making, 6(4), 333-342. 
Dibsdall, L., Lambert, N., Bobbin, R., \& Frewer, L. (2003). Low-income consumers' attitudes and behaviour towards access, availability and motivation to eat fruit and vegetables. Public Health Nutrition, 6(02), 159-168.

Diehl, K., \& Poynor, C. (2010). Great expectations?! Assortment size, expectations, and satisfaction. Journal of Marketing Research, 47(2), 312-322.

Diepeveen, S., Ling, T., Suhrcke, M., Roland, M., \& Marteau, T. M. (2013). Public acceptability of government intervention to change health-related behaviours: A systematic review and narrative synthesis. BMC public health, 13, 756. doi:10.1186/1471-2458-13-756

Doak, C. M., Visscher, T. L. S., Renders, C. M., \& Seidell, J. C. (2006). The prevention of overweight and obesity in children and adolescents: a review of interventions and programmes. Obesity reviews, 7(1), 111-136.

Dressler, H., \& Smith, C. (2013). Food choice, eating behavior, and food liking differs between lean/normal and overweight/obese, low-income women. Appetite, 65(0), 145-152. doi:10.1016/j.appet.2013.01.013

Drewnowski, A., \& Darmon, N. (2005). Food choices and diet costs: an economic analysis. Journal of Nutrition, 135(4), 900-904.

Drewnowski, A., Monsivais, P., Maillot, M., \& Darmon, N. (2007). Low-energydensity diets are associated with higher diet quality and higher diet costs in French adults. Journal of the American Dietetic Association, 107(6), 1028-1032.

Drewnowski, A., \& Specter, S. E. (2004). Poverty and obesity: The role of energy density and energy costs. American Journal Of Clinical Nutrition, 79(1), 616.

Duval, K., Strychar, I., Cyr, M.-J., Prud'homme, D., Rabasa-Lhoret, R., \& Doucet, É. (2008). Physical activity is a confounding factor of the relation between eating frequency and body composition. American Journal Of Clinical Nutrition, 88(5), 1200-1205.

Elbel, B. (2011). Consumer estimation of recommended and actual calories at fast food restaurants. Obesity, 19(10), 1971-1978. doi:10.1038/oby.2011.214

Epstein, L. H., Dearing, K. K., Roba, L. G., \& Finkelstein, E. (2010). The influence of taxes and subsidies on energy purchased in an experimental purchasing study. Psychological Science, 21(3), 406-414.

doi:10.1177/0956797610361446

Eriksson, L., Garvill, J., \& Nordlund, A. M. (2008). Acceptability of single and combined transport policy measures: The importance of environmental and policy specific beliefs. Transportation Research, 42(8), 1117-1128. doi:10.1016/j.tra.2008.03.006

Faulkner, G. E. J., Grootendorst, P., Nguyen, V. H., Andreyeva, T., ArbourNicitopoulos, K., Auld, M. C., ... Windmeijer, F. (2011). Economic instruments for obesity prevention: results of a scoping review and modified delphi survey. International Journal of Behavioral Nutrition and Physical Activity, 8(1), 1. doi:10.1186/1479-5868-8-109 
Finucane, M. M., Stevens, G. A., Cowan, M. J., Danaei, G., Lin, J. K., Paciorek, C. J., . . . Ezzati, M. (2011). National, regional, and global trends in body-mass index since 1980: Systematic analysis of health examination surveys and epidemiological studies with 960 country-years and 9.1 million participants. The Lancet, 377(9765), 557-567. doi:10.1016/s01406736(10)62037-5

Fornell, C., \& Larcker, D. F. (1981). Evaluating structural equation models with unobservable variables and measurement error. Journal of Marketing Research, 39-50.

French, S. A., Hannan, P. J., Harnack, L. J., Mitchell, N. R., Toomey, T. L., \& Gerlach, A. (2010). Pricing and availability intervention in vending machines at four bus garages. Journal of Occupational and Environmental Medicine, 52(Suppl 1), S29. doi:10.1097/JOM.0b013e3181c5c476

French, S. A., Jeffery, R. W., Story, M., Breitlow, K. K., Baxter, J. S., Hannan, P., \& Snyder, M. P. (2001). Pricing and promotion effects on low-fat vending snack purchases: The CHIPS Study. American Journal of Public Health, 91(1), 112-117.

French, S. D., Green, S. E., O'Connor, D. A., McKenzie, J. E., Francis, J. J., Michie, S., . .. Grimshaw, J. M. (2012). Developing theory-informed behaviour change interventions to implement evidence into practice: a systematic approach using the Theoretical Domains Framework. [Implementation Science C7 38]. Implementation Science, 7(1), 1-8. doi:10.1186/1748-5908-7-38

Gärling, T., \& Loukopoulos, P. (2007). Effectiveness, public acceptance, and political feasibility of coercive measures for reducing car traffic Threats from car traffic to the quality of urban life: Problems, causes, and solutions. (pp. 313-325). Amsterdam; The Netherlands: Elsevier.

Geeroms, N., Verbeke, W., \& Van Kenhove, P. (2008a). Consumers' health-related motive orientations and ready meal consumption behaviour. Appetite, 51(3), 704-712.

Geeroms, N., Verbeke, W., \& Van Kenhove, P. (2008b). Health advertising to promote fruit and vegetable intake: Application of health-related motive segmentation. Food Quality and Preference, 19(5), 481-497. doi:10.1016/j.foodqual.2008.02.004

Gendall, P., Hoek, J., Taylor, R., Mann, J., Krebs, J., \& Parry-Strong, A. (2015). Should support for obesity interventions or perceptions of their perceived effectiveness shape policy? Australian and New Zealand journal of public health, 39(2), 172-176.

Giesen, J. C. A. H., Havermans, R. C., Nederkoorn, C., \& Jansen, A. (2012). Impulsivity in the supermarket. Responses to calorie taxes and subsidies in healthy weight undergraduates. Appetite, 58(1), 6-10.

Giesen, J. C. A. H., Payne, C. R., Havermans, R. C., \& Jansen, A. (2011). Exploring how calorie information and taxes on high-calorie foods influence lunch decisions. American Journal Of Clinical Nutrition, 93(4), 689-694. doi:10.3945/ajen.110.008193 
Giskes, K., Van Lenthe, F., Avendano-Pabon, M., \& Brug, J. (2011). A systematic review of environmental factors and obesogenic dietary intakes among adults: Are we getting closer to understanding obesogenic environments? Obesity reviews, 12(501), e95-e106. doi:10.1111/j.1467789X.2010.00769.x

Giskes, K., Van Lenthe, F. J., Brug, J., Mackenbach, J. P., \& Turrell, G. (2007). Socioeconomic inequalities in food purchasing: The contribution of respondent-perceived and actual (objectively measured) price and availability of foods. Preventive Medicine, 45(1), 41-48. doi:10.1016/j.ypmed.2007.04.007

Gordon, R., McDermott, L., Stead, M., \& Angus, K. (2006). The effectiveness of social marketing interventions for health improvement: What's the evidence? Public Health, 120(12), 1133-1139. doi:10.1016/j.puhe.2006.10.008

Gortmaker, S. L., Swinburn, B. A., Levy, D., Carter, R., Mabry, P. L., Finegood, D. T., . .. Moodie, M. L. (2011). Changing the future of obesity: science, policy, and action. The Lancet, 378(9793), 838-847.

Gracia, A., \& Albisu, L. M. (2001). Food consumption in the European Union: Main determinants and country differences. Agribusiness, 17(4), 469-488. doi:10.1002/agr.1030

Grech, A., \& Allman-Farinelli, M. (2015). A systematic literature review of nutrition interventions in vending machines that encourage consumers to make healthier choices. Obesity reviews, 16(12), 1030-1041. doi:10.1111/obr.12311

Greene, G. W., Rossi, S. R., Rossi, J. S., Velicer, W. F., Fava, J. L., \& Prochaska, J. O. (1999). Dietary applications of the stages of change model. Journal of the American Dietetic Association, 99(6), 673-678. doi:10.1016/S00028223(99)00164-9

Greener, J., Douglas, F., \& Van Teijlingen, E. (2010). More of the same? Conflicting perspectives of obesity causation and intervention amongst overweight people, health professionals and policy makers. Social Science \& Medicine, 70(7), 1042-1049. doi:10.1016/j.socscimed.2009.11.017

Gregori, D., Foltran, F., Ghidina, M., \& Berchialla, P. (2011). Understanding the influence of the snack definition on the association between snacking and obesity: A review. International Journal of Food Sciences and Nutrition, 62(3), 270-275. doi:10.3109/09637486.2010.530597

Grunert, K. G., \& Wills, J. M. (2007). A review of European research on consumer response to nutrition information on food labels. Journal of Public Health, 15(5), 385-399.

Guh, D. P., Zhang, W., Bansback, N., Amarsi, Z., Birmingham, C. L., \& Anis, A. H. (2009). The incidence of co-morbidities related to obesity and overweight: A systematic review and meta-analysis. BMC public health, 9(1), 1. doi:10.1186/1471-2458-9-88 
Gustafson, A. A., Sharkey, J., Samuel-Hodge, C. D., Jones-Smith, J., Folds, M. C., Cai, J. W., \& Ammerman, A. S. (2011). Perceived and objective measures of the food store environment and the association with weight and diet among low-income women in North Carolina. Public Health Nutrition, 14(6), 10321038. doi:10.1017/s1368980011000115

Hair, J. F. (2010). Multivariate Data Analysis: A Global Perspective: Pearson Education.

Hall, K. D., Sacks, G., Chandramohan, D., Chow, C. C., Wang, Y. C., Gortmaker, S. L., \& Swinburn, B. A. (2011). Obesity 3: Quantification of the effect of energy imbalance on bodyweight. The Lancet, 378(9793), 826-837.

Harris, J. L., Pomeranz, J. L., Lobstein, T., \& Brownell, K. D. (2009). A crisis in the marketplace: How food marketing contributes to childhood obesity and what can be done. Annual review of public health, 30, 211-225.

Hartman, H., Wadsworth, D. P., Penny, S., Van Assema, P., \& Page, R. (2013). Psychosocial determinants of fruit and vegetable consumption among students in a New Zealand university. Results of focus group interviews. Appetite, 65(0), 35-42. doi:10.1016/j.appet.2013.02.005

Hartmann, C., Siegrist, M., \& van der Horst, K. (2013). Snack frequency: associations with healthy and unhealthy food choices. Public Health Nutrition, 16(8), 1487-1496. doi:10.1017/s1368980012003771

Hayes, A. F. (2012). PROCESS: A versatile computational tool for observed variable mediation, moderation, and conditional process modeling.

Hearty, A., McCarthy, S., Kearney, J., \& Gibney, M. (2007). Relationship between attitudes towards healthy eating and dietary behaviour, lifestyle and demographic factors in a representative sample of Irish adults. Appetite, 48(1), 1-11.

Hilbert, A., Rief, W., \& Braehler, E. (2007). What determines public support of obesity prevention? Journal of Epidemiology and Community Health, 61(7), 585-590. doi:10.1136/jech.2006.050906

Hollywood, L. E., Cuskelly, G. J., O'Brien, M., McConnon, A., Barnett, J., Raats, M. M., \& Dean, M. (2013). Healthful grocery shopping. Perceptions and barriers. Appetite, 70, 119-126.

Holmila, M., Mustonen, H., Österberg, E., \& Raitasalo, K. (2009). Public opinion and community-based prevention of alcohol-related harms. Addiction Research \& Theory, 17(4), 360-371.

Horgen, K. B., \& Brownell, K. D. (2002). Comparison of price change and health message interventions in promoting healthy food choices. Health Psychology, 21(5), 505-512.

Jacobson, M. F., \& Brownell, K. D. (2000). Small taxes on soft drinks and snack foods to promote health. American Journal of Public Health, 90(6), 854.

Johnson, G. H., \& Anderson, G. H. (2010). Snacking definitions: Impact on interpretation of the literature and dietary recommendations. Critical Reviews in Food Science and Nutrition, 50(9), 848-871.

doi:10.1080/10408390903572479 
Jou, J., \& Techakehakij, W. (2012). International application of sugar-sweetened beverage (SSB) taxation in obesity reduction: Factors that may influence policy effectiveness in country-specific contexts. Health Policy, 107(1), 8390. doi:10.1016/j.healthpol.2012.05.011

Kass, N. E. (2001). An ethics framework for public health. American Journal of Public Health, 91(11), 1776-1782.

Kay, A. C., Jimenez, M. C., \& Jost, J. T. (2002). Sour grapes, sweet lemons, and the anticipatory rationalization of the status quo. Personality and Social Psychology Bulletin, 28(9), 1300-1312. doi:10.1177/01461672022812014

Kelly, B., Hughes, C., Chapman, K., Louie, J. C. Y., Dixon, H., Crawford, J., . . . Slevin, T. (2009). Consumer testing of the acceptability and effectiveness of frontof-pack food labelling systems for the Australian grocery market. Health Promotion International, 24(2), 120-129. doi:10.1093/heapro/dap012

Kesten, J. M., Griffiths, P. L., \& Cameron, N. (2011). A systematic review to determine the effectiveness of interventions designed to prevent overweight and obesity in pre-adolescent girls. Obesity reviews, 12(12), 997-1021. doi:10.1111/j.1467-789X.2011.00919.x

Kirk, S. F. L., Penney, T. L., \& McHugh, T. L. F. (2010). Characterizing the obesogenic environment: the state of the evidence with directions for future research. Obesity reviews, 11(2), 109-117. doi:10.1111/j.1467-789X.2009.00611.x

Kocken, P. L., Eeuwijk, J., Van Kesteren, N., Dusseldorp, E., Buijs, G., Bassa-Dafesh, Z., \& Snel, J. (2012). Promoting the purchase of low-calorie foods from school vending machines: A cluster-randomized controlled study. Journal of School Health, 82(3), 115-122.

Kocken, P. L., van Kesteren, N., Buijs, G., Snel, J., \& Dusseldorp, E. (2015). Students' beliefs and behaviour regarding low-calorie beverages, sweets or snacks: Are they affected by lessons on healthy food and by changes to school vending machines? Public Health Nutrition, 18(09), 1545-1553. doi:10.1017/S1368980014002985

Kolodinsky, J., \& Reynolds, T. (2009). Segmentation of overweight Americans and opportunities for social marketing. International Journal of Behavioral Nutrition and Physical Activity, 6(1), 1. doi:10.1186/1479-5868-6-13

Kotler, P., \& Keller, K. (2006). Marketing management: Upper Saddle River, New Jersey: Pearson Prentice Hall. Kwon.

Krukowski, R. A., McSweeney, J., Sparks, C., \& West, D. S. (2012). Qualitative study of influences on food store choice. Appetite, 59(2), 510-516.

Larson, N., \& Story, M. (2010). Are 'competitive foods' sold at school making our children fat? Health Affairs, 29(3), 430-435. doi:10.1377/hlthaff.2009.0716

Laurin, K., Kay, A. C., \& Fitzsimons, G. J. (2012). Reactance versus rationalization: Divergent responses to policies that constrain freedom. Psychological Science, 23(2), 205-209.

Levitsky, D. A., \& Pacanowski, C. R. (2012). Free will and the obesity epidemic. Public Health Nutrition, 15(1), 126-141. 
Lim, S. S., Vos, T., Flaxman, A. D., Danaei, G., Shibuya, K., Adair-Rohani, H., . . . Bryan-Hancock, C. (2012). A comparative risk assessment of burden of disease and injury attributable to 67 risk factors and risk factor clusters in 21 regions, 1990-2010: A systematic analysis for the Global Burden of Disease Study 2010. The Lancet, 380. doi:10.1016/s0140-6736(12)61766-8

Lobstein, T., \& Dibb, S. (2005). Evidence of a possible link between obesogenic food advertising and child overweight. Obesity reviews, 6(3), 203-208.

Lowe, M. R., Tappe, K. A., Butryn, M. L., Annunziato, R. A., Coletta, M. C., Ochner, C. N., \& Rolls, B. J. (2010). An intervention study targeting energy and nutrient intake in worksite cafeterias. Eating Behaviors, 11(3), 144-151. doi:10.1016/j.eatbeh.2010.01.002

Lucan, S. C., \& Mitra, N. (2012). Perceptions of the food environment are associated with fast-food (not fruit-and-vegetable) consumption: Findings from multi-level models. International Journal of Public Health, 57(3), 599608. doi:10.1007/s00038-011-0276-2

Lusk, J. L., \& Ellison, B. (2013). Who is to blame for the rise in obesity? Appetite, 68(0), 14-20. doi:10.1016/j.appet.2013.04.001

Maes, L., Van Cauwenberghe, E., Van Lippevelde, W., Spittaels, H., De Pauw, E., Oppert, J.-M., . . . De Bourdeaudhuij, I. (2012). Effectiveness of workplace interventions in Europe promoting healthy eating: A systematic review. European Journal of Public Health, 22(5), 677-683.

Magnusson, R. S. (2010). Obesity prevention and personal responsibility: the case of front-of-pack food labelling in Australia. BMC public health, 10. doi:10.1186/1471-2458-10-662

Mai, R., \& Hoffmann, S. (2012). Taste lovers versus nutrition fact seekers: How health consciousness and self-efficacy determine the way consumers choose food products. Journal of Consumer Behaviour, 11(4), 316-328. doi:10.1002/cb.1390

Malik, V. S., Willett, W. C., \& Hu, F. B. (2013). Global obesity: Trends, risk factors and policy implications. Nature Reviews Endocrinology, 9(1), 13-27. doi:10.1038/nrendo.2012.199

Marcy, T. R., Britton, M. L., \& Harrison, D. (2011). Identification of barriers to appropriate dietary behavior in low-income patients with type 2 diabetes mellitus. Diabetes Therapy, 2(1), 9-19. doi:10.1007/s13300-010-0012-6

Mazzocchi, M., Cagnone, S., Bech-Larsen, T., Niedźwiedzka, B., Saba, A., Shankar, B., ... Traill, W. B. (2014). What is the public appetite for healthy eating policies? Evidence from a cross-European survey. Health Economics, Policy and Law, 10(3), 1-26. doi:10.1017/S1744133114000346

Meule, A., \& Gearhardt, A. (2014). Food addiction in the light of DSM-5. Nutrients, 6(9), 3653-3671.

Michie, S., Van Stralen, M. M., \& West, R. (2011). The behaviour change wheel: A new method for characterising and designing behaviour change interventions. Implementation Science, 6(1), 1-12. doi:10.1186/1748-59086-42 
Mikolajczak, J., Van den Berg, S. W., \& Bemelmans, W. J. E. (2012). Overweight prevention at secondary schools in 2010-2011: A nationwide follow-up study. RIVM.

Mitchell, G. L., Farrow, C., Haycraft, E., \& Meyer, C. (2013). Parental influences on children's eating behaviour and characteristics of successful parentfocussed interventions. Appetite, 60(0), 85-94. doi:10.1016/j.appet.2012.09.014

Mogilner, C., Rudnick, T., \& Iyengar, S. S. (2008). The mere categorization effect: how the presence of categories increases choosers' perceptions of assortment variety and outcome satisfaction. Journal of Consumer Research, 35(2), 202-215.

Monsivais, P., \& Drewnowski, A. (2007). The rising cost of low-energy-density foods. Journal of the American Dietetic Association, 107(12), 2071-2076. doi:10.1016/j.jada.2007.09.009

Mytton, O. T., Clarke, D., \& Rayner, M. (2012). Taxing unhealthy food and drinks to improve health. BMJ, 344. doi:10.1136/bmj.e2931

Nayga, R. M. (2008). Nutrition, obesity and health: policies and economic research challenges. European Review of Agricultural Economics, 35(3), 281-302. doi:10.1093/erae/jbn013

Nederkoorn, C., Havermans, R. C., Giesen, J. C. A. H., \& Jansen, A. (2011). High tax on high energy dense foods and its effects on the purchase of calories in a supermarket. An experiment. Appetite, 56(3), 760-765. doi:10.1016/j.appet.2011.03.002

Nestle, M., \& Jacobson, M. F. (2000). Halting the obesity epidemic: a public health policy approach. Public Health Reports, 115(1), 12-24.

Neumark-Sztainer, D., Story, M., Perry, C., \& Casey, M. A. (1999). Factors influencing food choices of adolescents: Findings from focus-group discussions with adolescents. Journal of the American Dietetic Association, 99(8), 929-937. doi:10.1016/s0002-8223(99)00222-9

Ni Mhurchu, C., Vandevijvere, S., Waterlander, W., Thornton, L. E., Kelly, B., Cameron, A. J., . . . Swinburn, B. A. (2013). Monitoring the availability of healthy and unhealthy foods and non-alcoholic beverages in community and consumer retail food environments globally. Obesity reviews, 14(S1), 108-119.

Nilsson, A., Schuitema, G., Jakobsson, C. B., Martinsson, J., \& Thorson, M. (2016). The road to acceptance: Attitude change before and after the implementation of a congestion tax. Journal of Environmental Psychology, 46, 1-9. doi:10.1016/j.jenvp.2016.01.011

Nuffield. (2007). Public health: Ethical issues (N. C. o. Bioethics Ed.). London: Nuffield Council on Bioethics.

Nunnally, J. C. (1978). Psychometric theory: McGraw-Hill.

O'Hara, B. J., Phongsavan, P., Eakin, E. G., Develin, E., Smith, J., Greenaway, M., \& Bauman, A. E. (2013). Effectiveness of Australia's Get Healthy Information and Coaching Service (R): maintenance of self-reported anthropometric 
and behavioural changes after program completion. BMC public health, 13, 175. doi:10.1186/1471-2458-13-175

Oliver, J. E., \& Lee, T. (2005). Public opinion and the politics of obesity in America. Journal of Health Politics Policy and Law, 30(5), 923-954. doi:10.1215/03616878-30-5-923

Ovaskainen, M. L., Reinivuo, H., Tapanainen, H., Hannila, M. L., Korhonen, T., \& Pakkala, H. (2005). Snacks as an element of energy intake and food consumption. European Journal of Clinical Nutrition, 60(4), 494-501.

Paisley, C. M., \& Sparks, P. (1998). Expectations of reducing fat intake: The role of perceived need within the theory of planned behaviour. Psychology \& Health, 13(2), 341-353. doi:10.1080/08870449808406755

Pan, A., Malik, V., \& Hu, F. B. (2012). Exporting diabetes to Asia: The impact of western-style fast food. Circulation. doi:10.1161/circulationaha.112.115923

Parker, D., Manstead, A. S. R., \& Stradling, S. G. (1995). Extending the theory of planned behaviour: The role of personal norm. British Journal of Social Psychology, 34(2), 127-138. doi:10.1111/j.2044-8309.1995.tb01053.x

Paul-Ebhohimhen, V., \& Avenell, A. (2008). Systematic review of the use of financial incentives in treatments for obesity and overweight. Obesity reviews, 9(4), 355-367. doi:10.1111/j.1467-789X.2007.00409.x

Pearl, R. L., \& Lebowitz, M. S. (2014). Beyond personal responsibility: Effects of causal attributions for overweight and obesity on weight-related beliefs, stigma, and policy support. Psychology \& Health, 1-29. doi:10.1080/08870446.2014.916807

Pechey, R., Burge, P., Mentzakis, E., Suhrcke, M., \& Marteau, T. M. (2014). Public acceptability of population-level interventions to reduce alcohol consumption: A discrete choice experiment. Social Science \& Medicine, 113(0), 104-109. doi:10.1016/j.socscimed.2014.05.010

Pomerleau, J., Lock, K., Knai, C., \& McKee, M. (2005). Interventions designed to increase adult fruit and vegetable intake can be effective: a systematic review of the literature. The Journal of Nutrition, 135(10), 2486-2495.

Pope, C., Ziebland, S., \& Mays, N. (2000). Analysing qualitative data. BMJ, 320, 114116.

Popkin, B. M., \& Duffey, K. J. (2010). Does hunger and satiety drive eating anymore? Increasing eating occasions and decreasing time between eating occasions in the United States. American Journal Of Clinical Nutrition, 91(5), 1342-1347. doi:10.3945/ajcn.2009.28962

Powell, L. M., Chriqui, J., Khan, T., Wada, R., \& Chaloupka, F. J. (2013). Assessing the potential effectiveness of food and beverage taxes and subsidies for improving public health: a systematic review of prices, demand and body weight outcomes. Obesity reviews, 14(2), 110-128. doi:10.1111/obr.12002

Powell, L. M., Szczypka, G., Chaloupka, F. J., \& Braunschweig, C. L. (2007). Nutritional content of television food advertisements seen by children and 
adolescents in the united states. Pediatrics, 120(3), 576-583.

doi:10.1542/peds.2006-3595

Promberger, M., Brown, R. C. H., Ashcroft, R. E., \& Marteau, T. M. (2011).

Acceptability of financial incentives to improve health outcomes in UK and US samples. Journal of Medical Ethics, 37, 682-687.

doi:10.1136/jme.2010.039347

Promberger, M., Dolan, P., \& Marteau, T. M. (2012). "Pay them if it works":

Discrete choice experiments on the acceptability of financial incentives to change health related behaviour. Social Science \& Medicine, 75(12), 2509 2514. doi:10.1016/j.socscimed.2012.09.033

Proudfoot, D., \& Kay, A. C. (2014). Reactance or rationalization? Predicting public responses to government policy. Policy Insights from the Behavioral and Brain Sciences, 1(1), 256-262. doi:10.1177/2372732214550489

Renner, B., Sproesser, G., Strohbach, S., \& Schupp, H. T. (2012). Why we eat what we eat. The Eating Motivation Survey (TEMS). Appetite, 59(1), 117-128.

Ronteltap, A., Sijtsema, S. J., Dagevos, H., \& de Winter, M. A. (2012). Construal levels of healthy eating: Exploring consumers' interpretation of health in the food context. Appetite, 59(2), 333-340.

Rosenstock, I. M., Strecher, V. J., \& Becker, M. H. (1988). Social learning theory and the health belief model. Health Education \& Behavior, 15(2), 175-183. doi:10.1177/109019818801500203

Rosseel, Y. (2012). Lavaan: An R package for structural equation modeling. Journal of Statistical Software, 48(2), 1-36.

Rothschild, M. L. (1999). Carrots, sticks, and promises: A conceptual framework for the management of public health and social issue behaviors. Journal of Marketing, 63(4), 24-37. doi:10.2307/1251972

Rozin, P., Scott, S., Dingley, M., Urbanek, J. K., Jiang, H., \& Kaltenbach, M. (2011). Nudge to nobesity I: Minor changes in accessibility decrease food intake. Judgment and Decision Making, 6(4), 323.

Sallis, J. F., Owen, N., \& Fisher, E. B. (2008). Ecological models of health behavior. Health behavior and health education: Theory, research, and practice, 4, 465-486.

Satia, J. A., Kristal, A. R., Curry, S., \& Trudeau, E. (2001). Motivations for healthful dietary change. Public Health Nutrition, 4(5), 953-959. doi:10.1079/phn2001157

Schaller, B. (2010). New York City's congestion pricing experience and implications for road pricing acceptance in the United States. Transport Policy, 17(4), 266-273.

Scholtens, S., Middelbeek, L., Rutz, S. I., Buijs, G., \& Bemelmans, W. J. (2010). Differences in school environment, school policy and actions regarding overweight prevention between Dutch schools. A nationwide survey. BMC public health, 10(1), 42. 
Schuitema, G., Steg, L., \& Van Kruining, M. (2011). When are transport pricing policies fair and acceptable? Social Justice Research, 24(1), 66-84. doi:10.1007/s11211-011-0124-9

Seiders, K., \& Petty, R. D. (2004). Obesity and the Role of Food Marketing: A Policy Analysis of Issues and Remedies. Journal of Public Policy \& Marketing, 23(2), 153-169. doi:doi:10.1509/jppm.23.2.153.51406

Seltman, H. J. (2012). Experimental design and analysis: Online at: http://www.stat.cmu.edu/hseltman/309/Book/Book.pdf.

Sheeshka, J. D., Woolcott, D. M., \& Mackinnon, N. J. (1993). Social cognitive theory as a framework to explain intentions to practice healthy eating behaviors. Journal of Applied Social Psychology, 23(19), 1547-1573. doi:10.1111/j.1559-1816.1993.tb01047.x

Shelley, J. J. (2012). Addressing the policy cacophony does not require more evidence: an argument for reframing obesity as caloric overconsumption. BMC public health, 12, 1042. doi:1042.10.1186/1471-2458-12-1042

Shnayder, L., Van Rijnsoever, F. J., \& Hekkert, M. P. (2015). Putting your money where your mouth is: Why sustainability reporting based on the triple bottom line can be misleading. PLoS ONE, 10(3), e0119036.

Sobal, J., \& Bisogni, C. A. (2009). Constructing food choice decisions. Annals of Behavioral Medicine, 38, S37-S46. doi:10.1007/s12160-009-9124-5

Sobel, M. E. (1982). Asymptotic confidence intervals for indirect effects in structural equation models. Sociological methodology, 13(1982), 290-312.

Steg, L., Dreijerink, L., \& Abrahamse, W. (2005). Factors influencing the acceptability of energy policies: A test of VBN theory. Journal of Environmental Psychology, 25(4), 415-425. doi:10.1016/j.jenvp.2005.08.003

Steg, L., Dreijerink, L., \& Abrahamse, W. (2006). Why are energy policies acceptable and effective? Environment and Behavior, 38(1), 92-111. doi:10.1177/0013916505278519

Steg, L., \& Vlek, C. (2009). Encouraging pro-environmental behaviour: An integrative review and research agenda. Journal of Environmental Psychology, 29(3), 309-317.

Stewart, D. W., \& Shamdasani, P. N. (1990). Focus groups: Theory and practice. Thousand Oaks, CA US: Sage Publications, Inc.

Stuckler, D., McKee, M., Ebrahim, S., \& Basu, S. (2012). Manufacturing epidemics: The role of global producers in increased consumption of unhealthy commodities including processed foods, alcohol, and tobacco. PLoS medicine, 9(6), 695.

Swinburn, B. A., Egger, G., \& Raza, F. (1999). Dissecting obesogenic environments: The development and application of a framework for identifying and prioritizing environmental interventions for obesity. Preventive Medicine, 29(6), 563-570. doi:10.1006/pmed.1999.0585 
Swinburn, B. A., Sacks, G., Hall, K. D., McPherson, K., Finegood, D. T., Moodie, M. L., \& Gortmaker, S. L. (2011). Obesity 1: The global obesity pandemic: shaped by global drivers and local environments. The Lancet, 378(9793), 804-814.

Ten Have, M., De Beaufort, I. D., Teixeira, P. J., Mackenbach, J. P., \& Van der Heide, A. (2011). Ethics and prevention of overweight and obesity: an inventory. Obesity reviews, 12(9), 669-679. doi:10.1111/j.1467-789X.2011.00880.x

Thornton, L. E., Cameron, A. J., McNaughton, S. A., Waterlander, W. E., Sodergren, M., Svastisalee, C., . . . Carter, M. (2013). Does the availability of snack foods in supermarkets vary internationally? International Journal of Behavioral Nutrition and Physical Activity, 10(1), 1.

Traill, W. B., Shankar, B., Brambila-Macias, J., Bech-Larsen, T., Aschemann-Witzel, J., Strand, M., . . L Lyle, D. (2010). Interventions to promote healthy eating habits: evaluation and recommendations. Obesity reviews, 11(12), 895898. doi:10.1111/j.1467-789X.2010.00717.x

Trudeau, E., Kristal, A. R., Li, S., \& Patterson, R. E. (1998). Demographic and psychosocial predictors of fruit and vegetable intakes differ: Implications for dietary interventions. Journal of the American Dietetic Association, 98(12), 1412-1417. doi:10.1016/s0002-8223(98)00319-8

Tukey, J. W. (1962). The future of data analysis. The Annals of Mathematical Statistics, 1-67.

Vallgarda, S., Holm, L., \& Jensen, J. D. (2015). The Danish tax on saturated fat: Why it did not survive. European Journal of Clinical Nutrition, 69(2), 223-226.

Van Kleef, E., Otten, K., \& Van Trijp, H. C. M. (2012). Healthy snacks at the checkout counter: a lab and field study on the impact of shelf arrangement and assortment structure on consumer choices. BMC public health, 12(1), 1072.

Van Rijnsoever, F. J., Van Lente, H., \& Van Trijp, H. C. M. (2011). Systemic policies towards a healthier and more responsible food system. Journal of Epidemiology and Community Health, 65(9), 737-739. doi:10.1136/jech.2011.141598

Van Trijp, H. C. M. (2010). Social marketing approaches to obesity prevention. In D. Crawford (Ed.), Obesity Epidemiology (2 ed.). Oxford; UK: Oxford University Press.

Van Trijp, H. C. M., Brug, J., \& Van der Maas, R. (2005). Consumer determinants and intervention strategies for obesity prevention. In D. J. Mela (Ed.), Food, Diet and Obesity. Cambridge; UK: Woodhead Publishing Ltd.

Ventura, A. K., \& Mennella, J. A. (2011). Innate and learned preferences for sweet taste during childhood. Current Opinion in Clinical Nutrition \& Metabolic Care, 14(4), 379-384. doi:10.1097/MCO.0b013e328346df65

Verweij, L. M., Coffeng, J., van Mechelen, W., \& Proper, K. I. (2011). Meta-analyses of workplace physical activity and dietary behaviour interventions on weight outcomes. Obesity reviews, 12(6), 406-429. doi:10.1111/j.1467789X.2010.00765.x 
Walsh, R. A., Paul, C. L., Tzelepis, F., Stojanovski, E., \& Tang, A. (2008). Is government action out-of-step with public opinion on tobacco control? Results of a New South Wales population survey. Australian and New Zealand journal of public health, 32(5), 482-488.

Waterlander, W. E., Steenhuis, I. H. M., De Vet, E., Schuit, A. J., \& Seidell, J. C. (2010). Expert views on most suitable monetary incentives on food to stimulate healthy eating. European Journal of Public Health, 20(3), 325331.

WHO. (2014). Global status report on non communicable diseases 2014. Retrieved from Geneva; Switzerland:

Wiecha, J. L., Finkelstein, D., Troped, P. J., Fragala, M., \& Peterson, K. E. (2006). School vending machine use and fast-food restaurant use are associated with sugar-sweetened beverage intake in youth. Journal of the American Dietetic Association, 106(10), 1624-1630. doi:10.1016/j.jada.2006.07.007

Withrow, D., \& Alter, D. A. (2011). The economic burden of obesity worldwide: a systematic review of the direct costs of obesity. Obesity reviews, 12(2), 131-141. doi:10.1111/j.1467-789X.2009.00712.x

Zizza, C., Siega-Riz, A. M., \& Popkin, B. M. (2001). Significant increase in young adults' snacking between 1977-1978 and 1994-1996 represents a cause for concern! Preventive Medicine, 32(4), 303-310.

doi:10.1006/pmed.2000.0817 


\section{SUMMARY}

The need for more effective interventions to combat the obesity problem has been expressed by many public health experts. While consumer support is important for intervention effectiveness, little is known about why consumers accept or do not accept food choice interventions. The present thesis therefore aims to examine the process underlying acceptance of intervention strategies for healthy food choices. Particularly the role that intervention characteristics (e.g. the source and the location of an intervention) and individual characteristics (i.e. consumers' perceived barriers for behaviour) play in this process is investigated. Three research questions are formulated: 1) What are the drivers of consumer acceptance of intervention strategies for healthy food choices?; 2) How do consumers' barriers for healthy food choices influence both food choices in real-life and acceptance of interventions?; 3) How does intervention intrusiveness influence acceptance and effectiveness of intervention strategies for healthy food choices?

General beliefs about the obesity problem and intervention-specific beliefs are identified as the main drivers of intervention acceptance in the food domain (Chapter 2). General beliefs comprised both people's ideas about the magnitude of the obesity problem and the responsibility for food choices that consumers make. Intervention-specific beliefs, on the other hand, concerned people's perceptions of whether an intervention will be effective, both personally and societally, in stimulating healthy food choices (perceived effectiveness) as well as whether the intervention is a fair way of stimulating healthier choices (perceived fairness).

Consumers' motivation-related barriers are associated strongest with real-life food choices, with higher levels of motivation to choose healthy foods being related to higher proportions of healthy choices (Chapter $\mathbf{3}$ ). Barriers related to the perceived opportunity and ability to choose healthy foods are associated less strongly with real-life choices, with higher levels of opportunity and ability being related to higher proportions of healthy food choices. Three distinct barrier profiles regarding choosing healthy foods are identified: the no-barrier profile (consisting of those who perceive to have no barriers), the lack-of-opportunity profile (consisting of those who perceive to lack opportunity), and the lack-of-motivation profile (consisting of those who report not being motivated). Consumers with a no-barrier profile or a lack-of-opportunity profile perceive interventions to be more effective, more fair, and more acceptable than those with a lack-of-motivation profile (Chapter $\mathbf{4}$ and $\mathbf{5}$ ). 
Acceptance of less intrusive interventions, such as calorie information provision, is higher than those of more intrusive interventions, such as elimination of unhealthy choices, both in hypothetical (Chapter 4) and realistic (Chapter 5) settings. In the hypothetical setting, the effect of intrusiveness on acceptance is mediated by both the perceived effectiveness and the perceived fairness of interventions: the more consumers perceive an intervention to be effective and fair, the more they accept it (Chapter 4). In the realistic setting, the effect of perceived intrusiveness on acceptance is mediated only by the perceived fairness of interventions (Chapter 5).

In a vending machine setting, the implementation of an intervention that eliminates $50 \%$ of unhealthy choices leads to higher proportions of healthy choices than the implementation of less intrusive interventions (providing calorie information through labelling and not intervening at all). Since acceptance does not differ between these interventions (Chapter 5), elimination of unhealthy choices appears to be the best way to stimulate consumers to make healthy choices.

Overall, this thesis show that consumers' general- and intervention-specific beliefs drive acceptance of intervention strategies for healthy food choices. Intervention intrusiveness partly influences acceptance, depending on the setting in which consumers are confronted with them (hypothetical vs. realistic). Furthermore, barriers that consumers perceive to have in relation to choosing healthy foods impact acceptance of interventions as well. These insights provide implications for policy makers, both in terms of the development and the communication of intervention strategies for healthy food choices. 


\section{DANKWOORD}

Tijdens de ruim vier jaar van mijn PhD heb ik veel nieuwe mensen leren kennen en, mede door deze mensen, nieuwe vaardigheden kunnen ontwikkelen, zowel op professioneel als persoonlijk vlak. Bij deze wil ik dan ook een aantal mensen eruit lichten.

Allereerst wil ik mijn team bedanken: mijn promotor Hans Van Trijp, en mijn copromotoren Ivo Van der Lans en Frank Van Rijnsoever. Hans, je bent enorm belangrijk geweest in het richting geven aan het onderzoek. Vooral het positioneren van papers en het bewaken van de consistente verhaallijn in deze papers was zonder jou niet zo soepel verlopen als nu het geval is geweest. Met je befaamde schema's schepte je tijdens onze meetings structuur in de brei van gedachten die zich in mijn hersenen ophielden. Ivo, jij bent mijn klankbord geweest. Je was altijd erg eerlijk over waar jij je waarde zag in het onderzoek en waar je vooral je 'gezonde verstand' gebruikte om me te adviseren. We hebben het naast gesprekken over het onderzoek ook vaak over 'de rest' van onze levens gehad. Over onze gemeenschappelijke sport tennis, over jouw vriendin en kinderen, over mijn onzekerheden, over mijn passie voor elektronica, en ga zo maar door. Ik ben je hier erg dankbaar voor en ik hoop dat we ook in de toekomst nog in contact blijven. Frank, jij zorgde met je achtergrond in Innovation Studies voor een complementair geluid binnen het project. Dit heeft een belangrijke bijdrage geleverd aan het genereren van onderzoeksideeën en het ontwerp van de onderzoeksmethodologie. Ik wil jullie alledrie enorm bedanken voor de verschillende manieren waarop jullie me hebben bijgestaan tijdens mijn PhD.

Daarnaast wil ik de leescommissie, bestaande uit Prof. Ellen Kampman, Prof. Koert Van Ittersum, Prof. Stef Kremers, en Dr Mario Mazzocchi, bedanken voor de tijd en inspanning die zij in het lezen van mijn proefschrift hebben gestoken.

Ook de mede-PhD's mogen niet in dit lijstje ontbreken. De uitstapjes, etentjes en borrels die we regelmatig ondernamen waren uitgelezen momenten om elkaar beter te leren kennen. En uiteraard om elkaar van tips te voorzien hoe je je PhD met zo weinig mogelijk kleerscheuren door kunt komen. In het bijzonder wil ik Roxanne en Aleksandra noemen, aangezien ze voor mij onlosmakelijk verbonden zijn met mijn jaren in Wageningen en daardoor mijn paranimfen zijn. Rox, terwijl we elkaar nauwelijks kenden zijn we 3 maanden nadat ik gestart was samen gaan wonen op de Haverlanden 99. Want een compleet huis met een tuin was toch veel comfortabeler 
dan een kamer van $11 \mathrm{~m}^{2}$ en $24 \mathrm{~m}^{2}$. Bijna 4 jaar hebben we er met heel veel plezier gewoond: we hebben vaak samen gegeten en gedronken, serieuze gesprekken gehad en hard gelachen. Het was echt ons thuis. In het laatste jaar kwam de liefde van je leven Angelo erbij in huis, en hoe mooi is het dat hij de cover van dit proefschrift heeft ontworpen. Angelo, geweldig bedankt hiervoor! En ja, Aleks, wat kan ik over jou zeggen? Ik had me geen betere collega, kamergenoot en maatje kunnen wensen. Al vanaf de eerste ontmoeting klikte het. We hebben elkaar heel goed leren kennen en elkaar er uiteindelijk ook echt doorheen gesleept. Wat me vooral zal bijblijven zijn de 473 keer dat we samen gegeten hebben, waarvan 63 keer bij Da Martini. Wat konden we toch heerlijk kletsen en ouwehoeren. Dat blijven we in de toekomst ook zeker doen!

En dan eindig ik met de mensen in mijn leven die me het meest dierbaar zijn. Pa en ma, bedankt voor alle steun die ik altijd van jullie heb mogen ontvangen. Voor de deur die altijd openstond en staat. Voor jullie interesse in mijn onderzoek, ook al was de inhoud ervan soms wat lastig te volgen. Voor het wassen van de vuile sportkleren die ik 'for old times' sake' nog wel eens langs bracht. Voor het gedogen van de grote hoeveelheid meuk in jullie huis. Jullie hebben het fantastisch gedaan de afgelopen 32 jaar. Dennis en Kevin, ik ben enorm blij en trots dat ik jullie als broers heb. Nu we een jaartje ouder (en softer) zijn, weten we elkaar nog meer te waarderen dan eerst. Zoveel wederzijds respect voor wat we hebben bereikt in ons leven, prachtig vind ik dat. En dit geldt uiteraard ook voor jullie fantastische vriendinnen Jeannette en Nynke, het is een genot om jullie in de familie te hebben.

En dan Jennie. Jij verdient eigenlijk een heel boek. Maar voor nu zal ik het kort houden. Wat is ons leven veranderd op 30 September 2015. Wat wij in elkaar hebben losgemaakt in zo'n korte tijd hadden we nooit voor mogelijk gehouden. De liefde die we voor elkaar voelen is zo puur en intens; daar kunnen we de rest van ons leven op teren. Ik kan het niet in woorden uitdrukken hoe dankbaar ik ben voor je enthousiasme, inzicht, relativeringsvermogen, werklust, reisdrang en positieve kijk op het leven. Zonder jou was ik niet waar ik nu ben. En geloof me, het wordt vanaf hier alleen nog maar mooier! 


\section{ABOUT THE AUTHOR}

Colin Bos was born in Joure (Friesland) on February $16^{\text {th }}, 1984$. He received his master's degree in Social Psychology at the University of Groningen in 2011. His thesis focused on the effects of repeated exposure to graphic fear appeals on cigarette packages. Interestingly, the Dutch government decided to introduce such graphic fear appeals a few months ago (May 2016).

Shortly after graduating, Colin started his PhD at the Wageningen University. As a member of the Marketing and Consumer Behaviour group, he started working on an NWO project that aimed to identify promising ways of stimulating healthy dietary behaviours. In this regard, Colin specifically focused on determinants of intervention acceptance from a consumer perspective. He presented his research at (inter)national conferences, and published several papers.

Currently Colin works at Philips Research in Eindhoven as a Research Scientist. He is involved in several projects that all aim to achieve the same goal: to make the world healthier and more sustainable through innovation. 
Colin Bos

Wageningen School of Social Sciences (WASS)

Completed Training and Supervision Plan

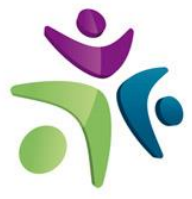

Wageningen School of Social Sciences
Name of the activity

Department/Institute

Year

ECTS

(1=28 hrs)

\section{A) Project related competences}

Research methodology: from topic to proposal

WASS

2011

4.0

PhD research proposal

MCB

'Understanding consumer acceptance of intervention strategies for healthy food choices: A qualitative study'

EHPS Conference

Bordeaux

'The effects of repeated exposure to graphic fear appeals in Smoker subgroups'

'Understanding consumer acceptance of intervention strategies for healthy food choices: A qualitative study'

EDEN Seminar Research Methods

WASS PhD day

2013

EDEN, Brussels

2014

4.0

$\mathrm{PhD}$ lunch colloquium series

MCB

2012-15

3.5

\section{B) General research related competences}

Philosophy and Ethics of Food Science and Technology Scientific Writing

VLAG/WGS

2013

WGS

2013

1.5
Introduction course

WASS

2011

2011

2012

WUR YRM 60806

Quantitative research methods: multivariate techniques, WUR YRM 60306

\section{C) Career-related competences}

\section{Career Perspectives}

Assisting MCB10806 \& MCB30806

WUR

2013

1.6

Effective behaviour in your professional surroundings

$\mathrm{MCB}$

2013

2.0

Supervising bachelor and master thesis student

WGS

2011

0.7

MCB 
Financing organization: The research described in this thesis was funded by NWO, the Netherlands Organization for Scientific Research, project number 2100882000.

Publisher: Digiforce / Proefschriftmaken.nl, Vianen

Cover design: Angelo Haemers 
o 